\title{
Medium-induced gluon radiation and colour decoherence beyond the soft approximation
}

\author{
Liliana Apolinário, ${ }^{a, b}$ Néstor Armesto, ${ }^{a}$ José Guilherme Milhano ${ }^{a, b, c}$ and \\ Carlos A. Salgado ${ }^{a}$
}

${ }^{a}$ Departamento de Física de Partículas and IGFAE, Universidade de Santiago de Compostela, 15706 Santiago de Compostela, Galicia-Spain

${ }^{b}$ CENTRA, Instituto Superior Técnico, Universidade de Lisboa, Av. Rovisco Pais, P-1049-001 Lisboa, Portugal

${ }^{c}$ Physics Department, Theory Unit, CERN, CH-1211 Genéve 23, Switzerland

E-mail: lilianamarisa.cunha@usc.es, nestor.armesto@usc.es, guilherme.milhano@tecnico.ulisboa.pt, carlos.salgado@usc.es

ABSTRACT: We derive the in-medium gluon radiation spectrum off a quark within the path integral formalism at finite energies, including all next-to-eikonal corrections in the propagators of quarks and gluons. Results are computed for finite formation times, including interference with vacuum amplitudes. By rewriting the medium averages in a convenient manner we present the spectrum in terms of dipole cross sections and a colour decoherence parameter with the same physical origin as that found in previous studies of the antenna radiation. This factorisation allows us to present a simple physical picture of the mediuminduced radiation for any value of the formation time, that is of interest for a probabilistic implementation of the modified parton shower. Known results are recovered for the particular cases of soft radiation and eikonal quark and for the case of a very long medium, with length much larger than the average formation times for medium-induced radiation. Technical details of the computation of the relevant $n$-point functions in colour space and of the required path integrals in transverse space are provided. The final result completes the calculation of all finite energy corrections for the radiation off a quark in a QCD medium that exist in the small angle approximation and for a recoilless medium.

KEYwords: Jets, Heavy Ion Phenomenology

ARXIV EPRINT: 1407.0599 


\section{Contents}

1 Introduction $\quad 2$

2 In-medium $q \longrightarrow q g$ splitting $\quad 3$

2.1 Quasi-eikonal in-medium parton propagation 3

2.2 Amplitudes 5

2.3 Emission cross section 6

3 Average over the medium ensemble $\quad 9$

3.1 Separation into regions 9

$\begin{array}{ll}3.2 & \text { Dipole approximation } \\ \end{array}$

4 Gluon emission spectrum 14

$\begin{array}{lll}4.1 & \text { Vacuum gluon spectrum } & 14\end{array}$

4.2 Medium emission spectrum 16

$\begin{array}{lll}5 & \text { Conclusions } & 17\end{array}$

$\begin{array}{ll}\text { A Dirac algebra } & 18\end{array}$

B Colour algebra $\quad 20$

B.1 Before region separation 20

$\begin{array}{lll}\text { B.2 After region separation } & 21\end{array}$

C Medium averages: $n$-point correlators of Wilson lines 22

$\begin{array}{ll}\text { C.1 Two-point correlation function } & 22\end{array}$

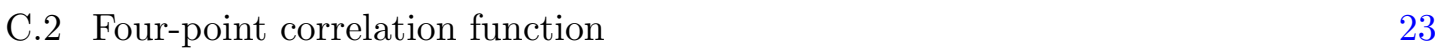

$\begin{array}{lll}\text { C.3 Six-point correlation function } & 27\end{array}$

D Path integrals in the multiple soft scattering approximation $\quad 29$

D.1 Semi-classical method and some general examples 30

D.2 Region I: two path integrals 32

D.3 Region II: three path integrals 33

D.4 Region III: four path integrals 34

$\begin{array}{lll}\text { E } & \text { BDMPS limit } & 38\end{array}$ 


\section{Introduction}

The dense and hot state of matter produced in heavy ion collisions, commonly referred to as a quark-gluon plasma (QGP), is characterised by the deconfinement of quarks and gluons up to distances much larger than the size of hadrons. Thus, it affords a window of opportunity to study QCD in a regime usually not accessible perturbatively. Given the very short lifetime of the QGP, only probes generated within, as part of the overall collision, can be used to determine its properties. Of particular importance are hard probes, produced in a hard scattering, which carry information about the initial stages of the collision and can be addressed within perturbation theory. The large centre-of-mass collision energy per nucleon pair in collider experiments - the Relativistic Heavy Ion Collider (RHIC) at BNL and, above all, the Large Hadron Collider (LHC) at CERN — leads to abundant hard particle production and, consequently, to the possibility of measuring a variety of observables with high statistics. Among such hard probes, those related to the modification of jets and jet-like properties of particle production resulting from the effects imparted by the hot and dense medium to the propagation dynamics of high-energy particles - what is commonly referred to as jet quenching, see e.g. [1,2] — provide the opportunity to extract detailed information about the medium.

Manifestations of jet quenching phenomena have been experimentally observed both for jet-like observables - e.g., in single inclusive particle spectra [3-7] and two-particle correlations [8-10] — and for fully reconstructed jets in $\mathrm{PbPb}$ collisions at the LHC [11-21] (see also $[22,23]$ for related results at RHIC). In particular, the observation of a sizeable increase, with respect to the proton-proton case, of the energy asymmetry in dijet systems without modification of their azimuthal correlation and for which the missing energy is recovered at large angles away from the jet axis in the form of soft particles, appeared, at first sight, to pose serious challenges for the standard explanation of jet quenching in terms of medium-induced gluon radiation in which energy loss and broadening are intrinsically connected. While several phenomenological explanations [24-33] have since been put forward, none provides a complete quantitative description of the data nor proper theoretical justification for some of the assumptions. This fact stresses the importance of having a faithful description of the mechanisms of energy loss so that a successful comparison with data can be done and the properties of the QGP deconvoluted from the confounding effects present in heavy-ion collisions. Such a requirement is particularly relevant for Monte Carlo implementations. While analytical models are currently derived within the high-energy approximation and usually implement energy-momentum conservation a posteriori, Monte Carlo generators encode it by construction. This implies further assumptions which lack a firm theoretical basis and often extend the use of theoretical models beyond their strict validity region [34]. In order to avoid this situation, finite energy corrections for the full radiation spectrum must be correctly (and fully) accounted for. Efforts in this direction include [35-37]. In this work we extend the results derived in [36], where an interpolation function between the soft and hard limits for gluon radiation off a quark was deduced, to account for an arbitrary momentum fraction to be carried by the radiated gluon.

The path-integral formalism [38] is used throughout. We allow transverse motion of all particles in the emission process, thus relaxing the common assumption that only the 
softest particle is allowed such movement. In an earlier work where the same constraint was removed [37], the gluon radiation off a gluon was computed assuming small formation times for the radiated gluon. By going beyond this approximation we not only present the full result of medium-induced gluon radiation at finite formation time and finite energy, but also a clear physical picture in which the relevant color coherence effects are identified. A future goal will be to convert this physical picture into a probabilistic approach suitable for Monte Carlo implementation. Let us also mention that while the region of validity of small formation times can be quite comfortable, it demands a large medium to be crossed by the radiating partons. This approximation will not hold for the ones that are emitted close to the edge of the medium.

Let us anticipate the main results of our work. We provide the most general form of a double inclusive spectrum, for gluon radiation off a fast quark in a QCD medium in the small angle approximation and for a recoilless medium. From it and in order to get expressions that can be used in practice, we make use of the Gaussian approximation for medium averages, work in the limit of large number of colours and employ the approximation of many soft scatterings, known as multiple soft or harmonic oscillator approximation. The last approximation allows an analytic solution of the path integral describing the noneikonal propagation of partons in the coloured medium - see [39] for an exact treatment and a comparison of different approximations using a scattering potential taken for thermal field theory at leading order in the coupling.

Our main qualitative result is the physical picture in terms of colour coherence/decoherence of the radiation process, with a factorization of dipole cross sections and a colour decoherence parameter with the same physical origin, but with some differences in the mathematical form, as the one found in the studies of the antenna radiation, $\Delta_{\text {med }}$ [40-44]. We find that colour coherence between the outgoing quark and gluon survives longer than in those calculations done in the eikonal limit for all propagators but the one of the softest particle, therefore suppressing the spectrum of radiated gluons. All these corrections are found to vanish in the limit of negligible formation time of the produced gluon, where we recover the results known in the literature, in particular those in [37].

The paper is organized as follows: in section 2, the formalism used to describe the inmedium propagation of partons is introduced, and the different contributions to the singlegluon emission spectrum for a static colour medium profile are calculated. The average over all possible colour configurations of the medium and generalisation of the decoherence parameter is carried out in section 3. The total radiation spectrum with the evaluation of the necessary path-integrals is presented in section 4, while the final conclusions are presented in section 5. Technical details are provided as appendices.

\section{$2 \quad$ In-medium $q \longrightarrow q g$ splitting}

\subsection{Quasi-eikonal in-medium parton propagation}

The time scale involved in the propagation of energetic partons is much smaller than the characteristic time for changes in the configuration of the medium that they traverse. This difference in time scales allows for the computation of the parton-medium interaction to 
be performed for a fixed, but arbitrary, medium configuration and, at a later stage, for the ensemble of medium configurations to be accounted for through an averaging procedure.

The multiple scattering of the propagating parton off medium components is mediated by the exchange of gluons with typical, purely transverse, momenta of the order of the characteristic medium scales. As a result, the otherwise eikonal trajectory of the parton the rotation of its colour phase without degradation of its (large) longitudinal momentum - is perturbed by Brownian motion in the transverse plane. The in-medium propagation of a parton with light-cone ${ }^{1}$ plus momentum $p_{+}$from transverse position $\mathbf{x}_{i}$ at time $x_{i+}$ (where its colour is $\alpha_{i}$ ) to transverse position $\mathbf{x}_{f}$ at time $x_{f+}$, with colour rotated to $\alpha_{f}$, is given by the Green's function

$$
\begin{aligned}
G_{\alpha_{f} \alpha_{i}}\left(x_{f+}, \mathbf{x}_{f} ; x_{i+}, \mathbf{x}_{i} \mid p_{+}\right)= & \int_{\mathbf{r}\left(x_{i+}\right)=\mathbf{x}_{i}}^{\mathbf{r}\left(x_{f+}\right)=\mathbf{x}_{f}} \mathcal{D} \mathbf{r}(\xi) \exp \left\{\frac{i p_{+}}{2} \int_{x_{i+}}^{x_{f+}} d \xi\left(\frac{d \mathbf{r}}{d \xi}\right)^{2}\right\} \\
& \times W_{\alpha_{f} \alpha_{i}}\left(x_{f+}, x_{i+} ; \mathbf{r}(\xi)\right)
\end{aligned}
$$

where the Wilson line

$$
W_{\alpha_{f} \alpha_{i}}\left(x_{f+}, x_{i+} ; \mathbf{r}(\xi)\right)=\mathcal{P} \exp \left\{i g \int_{x_{i+}}^{x_{f+}} d \xi A_{-}(\xi, \mathbf{r}(\xi))\right\}
$$

accounts for the colour rotation resulting from an arbitrary number of scatterings off the medium field $A_{-} \equiv A_{-}^{a} T^{a}$ ( $T^{a}$ being the colour matrix in the corresponding representation), while the free propagator

$$
\begin{aligned}
G_{0}\left(x_{f+}, \mathbf{x}_{f} ; x_{i+}, \mathbf{x}_{i} \mid p_{+}\right) & =\int_{\mathbf{r}\left(x_{i+}\right)=\mathbf{x}_{i}}^{\mathbf{r}\left(x_{f+}\right)=\mathbf{x}_{f}} \mathcal{D} \mathbf{r}(\xi) \exp \left\{\frac{i p_{+}}{2} \int_{x_{i+}}^{x_{f+}} d \xi\left(\frac{d \mathbf{r}}{d \xi}\right)^{2}\right\} \\
& =\frac{p_{+}}{2 \pi i\left(x_{f+}-x_{i+}\right)} \exp \left\{\frac{i p_{+}}{2} \frac{\left(\mathbf{x}_{f}-\mathbf{x}_{i}\right)^{2}}{x_{f+}-x_{i+}}\right\}
\end{aligned}
$$

encodes the random walk in the transverse plane. The Wilson line $W_{\alpha_{f} \alpha_{i}}$ in eq. (2.2), and consequently $G_{\alpha_{f} \alpha_{i}}$ in eq. (2.1), should be understood to carry colour indices in the relevant representation for the parton under consideration. In the following, fundamental colour indices, as relevant for propagating quarks, will be written in uppercase latin letters, while for the gluon the adjoint indices will be written in lowercase latin letters.

For compactness, and improved readability, we introduce the shorthand notation

$$
\begin{aligned}
G_{\alpha_{f} \alpha_{i}}\left(X_{f}, X_{i}, \mid p_{+}\right) & \equiv G_{\alpha_{f} \alpha_{i}}\left(x_{f+}, \mathbf{x}_{f} ; x_{i+}, \mathbf{x}_{i} \mid p_{+}\right) \\
& =\int_{X_{i}}^{X_{F}} \mathcal{D} \mathbf{r}(\xi) \exp \left\{\frac{i p_{+}}{2} \int_{x_{i+}}^{x_{f+}} d \xi\left(\frac{d \mathbf{r}}{d \xi}\right)^{2}\right\} W_{\alpha_{f} \alpha_{i}}(\mathbf{r})
\end{aligned}
$$

where $X_{f,(i)} \equiv\left(x_{f,(i)+}, \mathbf{x}_{f,(i)}\right)$.

\footnotetext{
${ }^{1}$ Light-cone coordinates, $a=\left(a_{0}, a_{x}, a_{y}, a_{z}\right)=\left(a_{+}, a_{-}, \mathbf{a}\right)$ with $a_{ \pm}=\left(a_{0} \pm a_{z}\right) / \sqrt{2}$ and transverse 2 -vectors $\mathbf{a}=\left(a_{x}, a_{y}\right)$, are used throughout.
} 


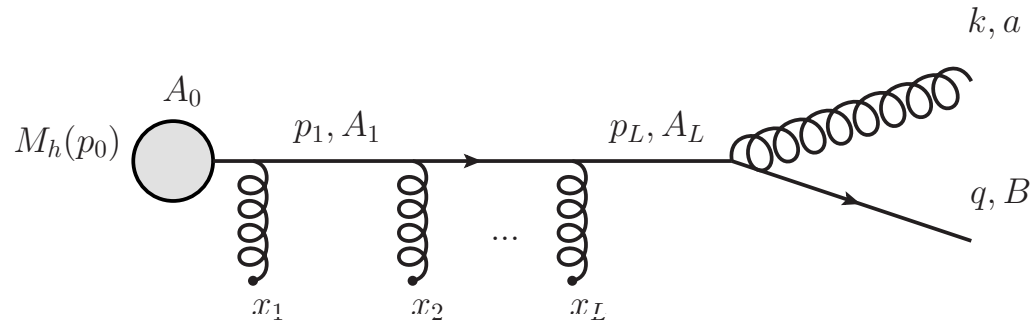

(a) $q \rightarrow q g$ splitting where only the initial particle interacts with the medium.

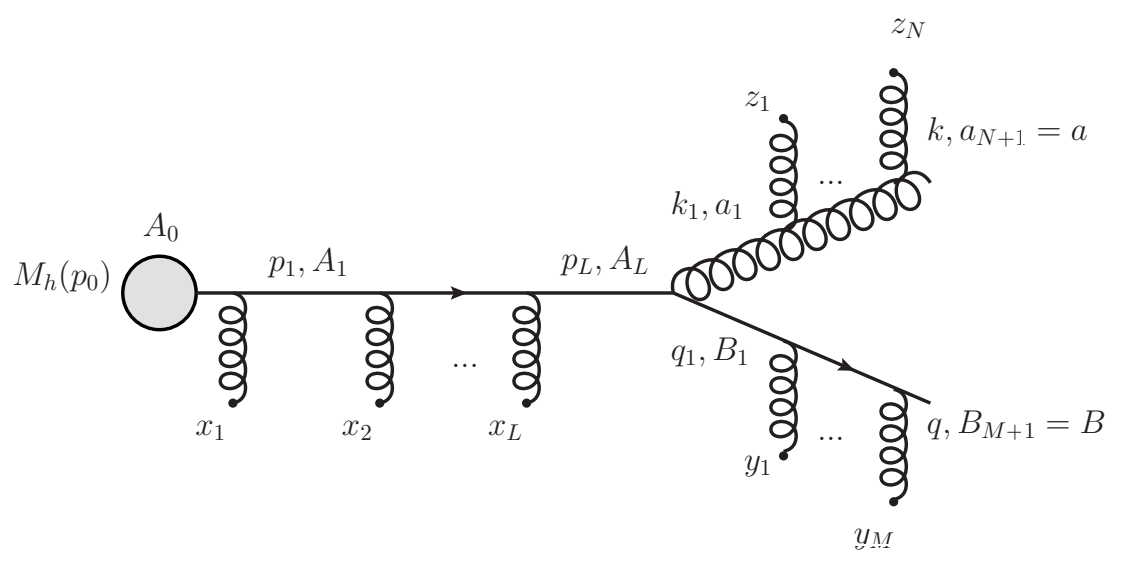

(b) $q \rightarrow q g$ splitting where all particles interact with the medium.

Figure 1: Diagrams that contribute to the in-medium $q \rightarrow q g$ splitting.

\section{$2.2 \quad$ Amplitudes}

To compute the radiation of a gluon off an energetic quark produced in a hard process in the early stages of a heavy-ion collision, two separate contributions to the amplitude ought to be considered: the case in which the splitting occurs outside a fixed length medium (see figure 1a) and thus only the initial quark experiences medium interactions; and the complementary situation in which the splitting occurs within the medium boundaries $\left(x_{0+}, L_{+}\right)$ (see figure 1b) and the interaction of all partons with the medium must be accounted for. ${ }^{2}$

The total amplitude can be written as

$$
\mathcal{T}_{\text {tot }}=\mathcal{T}_{\text {out }}+\mathcal{T}_{\text {in }}
$$

where the out and in contributions are given respectively by

$$
\begin{aligned}
\mathcal{T}_{\text {out }}= & \frac{-g}{2(k \cdot q)} T_{B A_{1}}^{a} \int_{-\infty}^{+\infty} d \mathbf{x} d \mathbf{x}_{0} \mathrm{e}^{-i \mathbf{x} \cdot(\mathbf{k}+\mathbf{q})+i \mathbf{x}_{0} \cdot \mathbf{p}_{0}} G_{A_{L} A_{0}}\left(X, X_{0} \mid p_{0+}\right) \\
& \times \bar{u}(q) \notin_{k}^{*}(\not k+q) \gamma_{+} M_{h}\left(p_{0}\right)(2 \pi) \delta\left(k+q-p_{0}\right)_{+}
\end{aligned}
$$

\footnotetext{
${ }^{2}$ We recall that the hard process, of amplitude $M_{h}$, from which the quark originates is unmodified by the surrounding environment since it occurs within a time/length scale too small to be resolved by the medium.
} 
and $^{3}$

$$
\begin{aligned}
\mathcal{T}_{\text {in }}= & \sum_{L, M, N} i g \int_{\mathbf{y}}^{y_{+}} \\
& \times \prod_{n=1}^{N} \int_{\mathbf{k}_{n}, \mathbf{z}_{n}}^{k_{n-}, z_{n+}} \mathrm{e}^{-i k_{n-}\left(z_{n}-z_{n-1}\right)_{+}+i \mathbf{k}_{n}\left(\mathbf{z}_{n}-\mathbf{z}_{n-1}\right)} \frac{2 i k_{+}}{k_{n}^{2}+i \varepsilon} i g A_{-a_{n+1} a_{n}}\left(z_{n+}, \mathbf{z}_{n}\right) \\
& \times \prod_{m=1}^{M} \int_{\mathbf{q}_{m}, \mathbf{y}_{m}}^{q_{m-}, y_{m+}} \mathrm{e}^{-i q_{m-}\left(y_{m}-y_{m-1}\right)_{+}+i \mathbf{q}_{m}\left(\mathbf{y}_{m}-\mathbf{y}_{m-1}\right)} \frac{2 i q_{+}}{q_{m}^{2}+i \varepsilon} i g A_{-B_{m+1} B_{m}}\left(y_{m+}, \mathbf{y}_{m}\right) \\
& \times T_{B_{1} A_{L}}^{a_{1}} \prod_{l=1}^{L} \int_{\mathbf{x}_{l}, \mathbf{p}_{l}}^{p_{l-}, x_{l+}} \mathrm{e}^{-i p_{l-}\left(x_{l+1}-x_{l}\right)_{+}+i \mathbf{p}_{l}\left(\mathbf{x}_{l+1}-\mathbf{x}_{l}\right)} \frac{2 i p_{+}}{p_{l}^{2}+i \varepsilon} i g A_{-A_{l} A_{l-1}}\left(x_{l+}, \mathbf{x}_{l}\right) \\
& \times \frac{1}{2 p_{+}} \frac{1}{2 q_{+}} \mathrm{e}^{-i \mathbf{z}_{N} \cdot \mathbf{k}-i \mathbf{y}_{M} \cdot \mathbf{q}+i \mathbf{x}_{1} \cdot \mathbf{p}_{0}} \\
& \times\left.\bar{u}(q) \gamma_{+} \not \phi_{1} \notin_{k_{1}}^{*} \not p_{L} \gamma_{+} M_{h}\left(p_{0}\right)(2 \pi) \delta\left(k+q-p_{0}\right)_{+}\right|_{z_{0}=y_{0}=x_{L+1}=y}
\end{aligned}
$$

where $X_{0}=\left(x_{0+}, \mathbf{x}_{0}\right)$ are the coordinates of the quark at the beginning of the medium and $X=\left(L_{+}, \mathbf{x}\right)$ the coordinates after the final scatterings.

\subsection{Emission cross section}

The cross section for single gluon emission is given by the average over the ensemble of medium configurations $\langle\ldots\rangle$ (to be carried out in section 3 )

$$
\frac{d^{2} \sigma}{d \Omega_{k} d \Omega_{q}}=\left\langle\left|\mathcal{T}_{\text {tot }}\right|^{2}\right\rangle
$$

with $d \Omega_{k}=(2 \pi)^{-3} d \mathbf{k} d k_{+} /\left(2 k_{+}\right)$and analogously for $d \Omega_{q}$. This inelastic cross section the squared amplitude averaged over initial spin and colour and summed over final spin, colour and gluon polarisation - is given by

$$
\left|\mathcal{T}_{\text {tot }}\right|^{2}=\left|\mathcal{T}_{\text {out }}\right|^{2}+\left|\mathcal{T}_{\text {in }}\right|^{2}+2 \operatorname{Re}\left\{\mathcal{T}_{\text {in }} \mathcal{T}_{\text {out }}^{\dagger}\right\}
$$

After simplification of both the Dirac algebra - for which details are given in appendix A - and the colour algebra - explicitly carried in appendix B.1 - the separate contributions on the right hand side of eq. (2.9) read:

$$
\begin{aligned}
\left|\mathcal{T}_{\text {out }}\right|^{2}= & g^{2} \frac{4 \sqrt{2} \zeta(1-\zeta) p_{0+}}{[(1-\zeta) \mathbf{k}-\zeta \mathbf{q}]^{2}} P_{g \leftarrow q}(\zeta) \int_{\mathbf{x}_{0}, \mathbf{x}, \overline{\mathbf{x}}_{0}, \overline{\mathbf{x}}} \mathrm{e}^{-i(\mathbf{k}+\mathbf{q}) \cdot(\mathbf{x}-\overline{\mathbf{x}})+i \mathbf{p}_{0} \cdot\left(\mathbf{x}_{0}-\overline{\mathbf{x}}_{0}\right)} \\
& \times \frac{1}{N} \operatorname{Tr}\left(G\left(X, X_{0} \mid p_{0+}\right) G^{\dagger}\left(\bar{X}, \bar{X}_{0} \mid p_{0+}\right)\right)\left|M_{h}\left(p_{0+}\right)\right|^{2}\left[2 \pi \delta\left(k+q-p_{0}\right)_{+}\right]^{2},
\end{aligned}
$$

\footnotetext{
${ }^{3}$ For compactness, we will use throughout the shorthands $\int_{-\infty}^{+\infty} d \mathbf{x} \equiv \int_{\mathbf{x}}$ and $\int_{x_{0+}}^{L_{+}} d x_{+} \equiv \int^{x_{+}}$.
} 


$$
\begin{aligned}
& \mathcal{T}_{\text {in }} \mathcal{T}_{\text {out }}^{\dagger}=g^{2} 2 \sqrt{2} \frac{1}{C_{F}} P_{g \leftarrow q}(\zeta) \int_{\mathbf{x}_{0}, \mathbf{x}_{1}, \mathbf{y}, \mathbf{z}, \overline{\mathbf{x}}_{0}, \overline{\mathbf{x}}}^{x_{1+}} \mathrm{e}^{-i \mathbf{k} \cdot(\mathbf{z}-\overline{\mathbf{x}})-i \mathbf{q} \cdot(\mathbf{y}-\overline{\mathbf{x}})+i \mathbf{p}_{0} \cdot\left(\mathbf{x}_{0}-\overline{\mathbf{x}}_{0}\right)} \\
& \times \frac{(1-\zeta) \mathbf{k}-\zeta \mathbf{q}}{[(1-\zeta) \mathbf{k}-\zeta \mathbf{q}]^{2}} \cdot\left((1-\zeta) \frac{\partial}{\partial \mathbf{z}_{1}}-\zeta \frac{\partial}{\partial \mathbf{y}_{1}}\right) \\
& \times \frac{1}{2 N}\left\{\int_{Z_{1}}^{Z} \mathcal{D} \boldsymbol{\omega}(\xi) \exp \left\{\frac{i k_{+}}{2} \int_{z_{1+}}^{L_{+}} d \xi \dot{\boldsymbol{\omega}}^{2}\right\} \operatorname{Tr}\left(G\left(Y, Y_{1} \mid q_{+}\right) W^{\dagger}(\boldsymbol{\omega})\right)\right. \\
& \times \operatorname{Tr}\left(G\left(X_{1}, X_{0} \mid p_{0+}\right) G^{\dagger}\left(\bar{X}, \bar{X}_{0} \mid p_{0+}\right) W(\boldsymbol{\omega})\right) . \\
& \left.-\frac{1}{N} \operatorname{Tr}\left(G\left(X_{1}, X_{0} \mid p_{0+}\right) G^{\dagger}\left(\bar{X}, \bar{X}_{0} \mid p_{0+}\right) G\left(Y, Y_{1} \mid q_{+}\right)\right)\right\}_{Z_{1}=Y_{1}=X_{1}} \\
& \times\left|M_{h}\left(p_{0+}\right)\right|^{2}\left[2 \pi \delta\left(k+q-p_{0}\right)_{+}\right]^{2}, \\
& \left|\mathcal{T}_{\text {in }}\right|^{2}=g^{2} \frac{\sqrt{2}}{\zeta(1-\zeta) p_{0+}} \frac{1}{C_{F}} P_{g \leftarrow q}(\zeta) \int_{\mathbf{x}_{0}, \mathbf{x}_{1}, \mathbf{y}, \mathbf{z}, \overline{\mathbf{x}}_{0}, \overline{\mathbf{x}}_{2}, \overline{\mathbf{y}}, \overline{\mathbf{z}}}^{x_{1+}, x_{2+}} \mathrm{e}^{-i \mathbf{k} \cdot(\mathbf{z}-\overline{\mathbf{z}})-i \mathbf{q} \cdot(\mathbf{y}-\overline{\mathbf{y}})+i \mathbf{p}_{0} \cdot\left(\mathbf{x}_{0}-\overline{\mathbf{x}}_{0}\right)} \\
& \times\left[\left((1-\zeta) \frac{\partial}{\partial \mathbf{z}_{1}}-\zeta \frac{\partial}{\partial \mathbf{y}_{1}}\right) \cdot\left((1-\zeta) \frac{\partial}{\partial \overline{\mathbf{z}}_{2}}-\zeta \frac{\partial}{\partial \overline{\mathbf{y}}_{2}}\right)\right] \frac{1}{2 N} \\
& \times\left\{\int_{Z_{1}}^{Z} \mathcal{D} \boldsymbol{\omega}(\xi) \exp \left\{\frac{i k_{+}}{2} \int_{z_{1+}}^{L_{+}} d \xi \dot{\boldsymbol{\omega}}^{2}\right\} \int_{\bar{Z}_{2}}^{\bar{Z}} \mathcal{D} \overline{\boldsymbol{\omega}}(\bar{\xi}) \exp \left\{\frac{-i k_{+}}{2} \int_{\bar{z}_{2+}}^{L_{+}} d \bar{\xi} \overline{\boldsymbol{\omega}}^{2}\right\}\right. \\
& \operatorname{Tr}\left(W^{\dagger}(\overline{\boldsymbol{\omega}}) W(\boldsymbol{\omega}) G\left(X_{1}, X_{0} \mid p_{0+}\right) G^{\dagger}\left(\bar{X}_{2}, \bar{X}_{0} \mid p_{0+}\right)\right) \\
& \operatorname{Tr}\left(G^{\dagger}\left(\bar{Y}, \bar{Y}_{2} \mid q_{+}\right) G\left(Y, Y_{1} \mid q_{+}\right) W^{\dagger}(\boldsymbol{\omega}) W(\overline{\boldsymbol{\omega}})\right) \\
& -\frac{1}{N} \operatorname{Tr}\left(G\left(X_{1}, X_{0} \mid p_{0+}\right) G^{\dagger}\left(\bar{X}_{2}, \bar{X}_{0} \mid p_{0+}\right) G^{\dagger}\left(\bar{Y}, \bar{Y}_{2} \mid q_{+}\right)\right. \\
& \left.\left.\times G\left(Y, Y_{1} \mid q_{+}\right)\right)\right\}_{\substack{Z_{1}=Y_{1}=X_{1} \\
\bar{Z}_{2}=\bar{Y}_{2}=\bar{X}_{2}}}\left|M_{h}\left(p_{0+}\right)\right|^{2}\left[2 \pi \delta\left(k+q-p_{0}\right)_{+}\right]^{2},
\end{aligned}
$$

where $P_{g \leftarrow q}(\zeta)=C_{F}\left[1+(1-\zeta)^{2}\right] / \zeta$ is the (vacuum) Altarelli-Parisi splitting function [45] with $\zeta$ the fraction of longitudinal momenta carried by the gluon (as defined in appendix A), $X_{1}=\left(x_{1+}, \mathbf{x}_{1}\right), Y_{1}=\left(y_{1+}, \mathbf{y}_{1}\right)$ and $Z_{1}=\left(z_{1+}, \mathbf{z}_{1}\right)$ the coordinates at the emission point for the initial quark, final quark and gluon ${ }^{4}$ respectively and $Y=\left(L_{+}, \mathbf{y}\right), Z=\left(L_{+}, \mathbf{z}\right)$, the coordinates after the final scatterings. It was necessary to introduce new coordinates for the complex conjugate amplitude, denoted by $\bar{X}_{i}\left(\bar{Y}_{i}, \bar{Z}_{i}\right)=\left(x_{i+}, \overline{\mathbf{x}}_{i}\left(\overline{\mathbf{y}}_{i}, \overline{\mathbf{z}}_{i}\right)\right)$ to represent the initial quark (final quark, gluon). The emission point in the complex conjugate amplitude is denoted by $x_{2+}$. The complete set of coordinates for the three contributions in eq. (2.9) is shown in figure 2 assuming $x_{2+}>x_{1+}$.

It should be noted that one of the transverse momenta from the Dirac structure of the in - out term, and all the ones in the in - in term, correspond to an internal component in the $\mathcal{T}_{\text {in }}$ amplitude. Therefore, they should be written as the derivative of the initial

\footnotetext{
${ }^{4}$ Although we use different emission coordinates, these are in fact the same $\left(\mathbf{x}_{1}=\mathbf{y}_{1}=\mathbf{z}_{1}\right)$.
} 


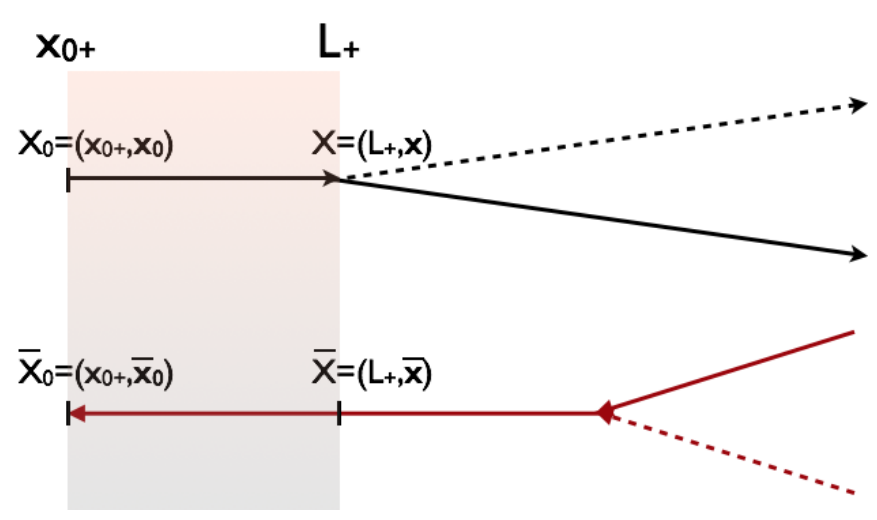

(a) Out-out contribution.

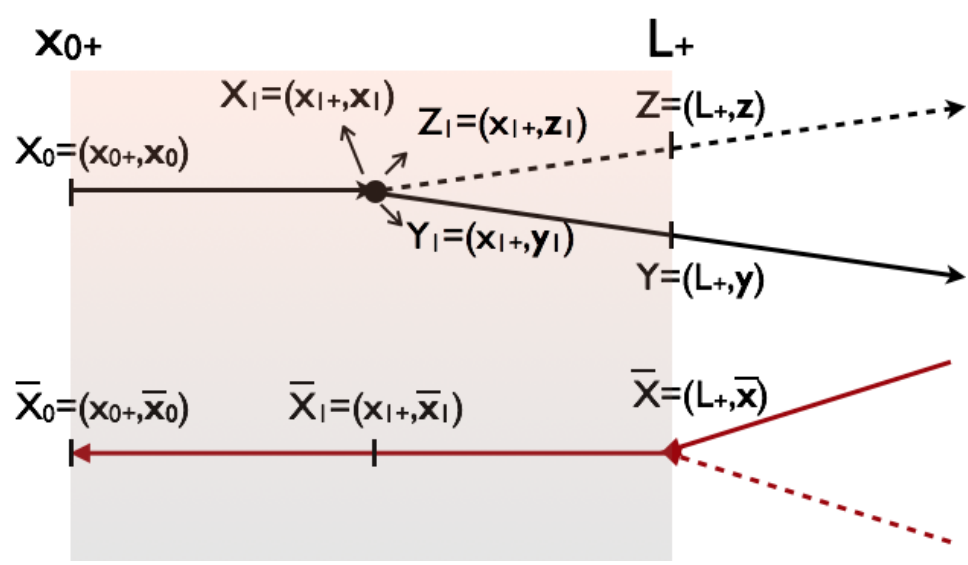

(b) In - out contribution.

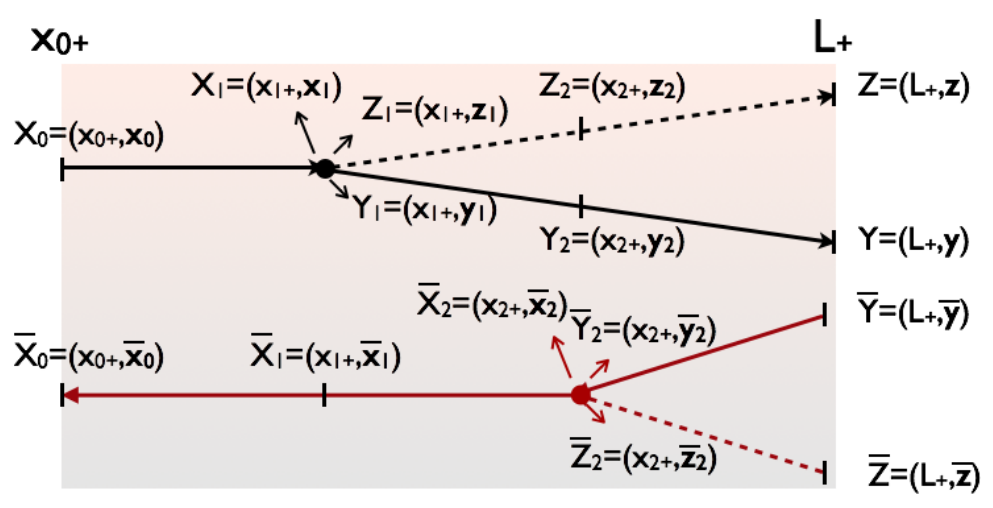

(c) In - in contribution.

Figure 2: Diagrams showing the coordinates used in the path integrals and Wilson lines in eqs. (2.10), (2.11) and (2.12). In each plot the full arrows represent the quarks and the dashed arrows the gluons. Black arrows hold for the amplitude and red arrows for the complex conjugate amplitude. The medium is shown by a coloured region that starts at $x_{0+}$ and ends at $L_{+}$. 
transverse coordinate of the corresponding Green's function at the vertex:

$$
\begin{aligned}
\mathbf{q}_{i} G\left(Y, Y_{i} \mid q_{+}\right) & =i \frac{\partial}{\partial \mathbf{y}_{\mathbf{i}}} G\left(Y, Y_{i} \mid q_{+}\right), \\
\mathbf{k}_{i} G\left(Z, Z_{i} \mid k_{+}\right) & =i \frac{\partial}{\partial \mathbf{z}_{\mathbf{i}}} G\left(Z, Z_{i} \mid k_{+}\right) .
\end{aligned}
$$

Note that the (vacuum) Altarelli-Parisi splitting function is recovered in all terms: out-out, $i n-o u t$ and $i n-i n$, as found in [37] and in previous works [46-48] for the energy spectrum.

\section{Average over the medium ensemble}

The squared matrix elements - eqs. (2.10), (2.11), and (2.12) - have been calculated for a fixed, but arbitrary, medium colour configuration in which the propagation and emission processes take place. In order to account for the ensemble of possible medium colour configurations an averaging procedure is carried out. Since all medium information is encoded in the Wilson lines $W$, where all colour information resides, the averaging procedure amounts to the evaluation of correlators of Wilson lines (several examples are listed in appendix C). The longitudinal locality of the interactions with the medium, a consequence of the high-energy approximation, allows for the decomposition of the longitudinal support of each squared matrix element into regions with a constant number of Wilson lines. Recalling that the colour structure and transverse momentum dynamics (i.e. the random walk in transverse plane) are factorised, see eq. (2.1), together with the convolution properties of the $G$ 's, it is possible to separate a propagator at an arbitrary point within a given longitudinal support. That is to say, that a Green's function with longitudinal support in the interval $\left(x_{i+}, x_{f+}\right)$ can be separated at a longitudinal location $x_{i+}<x_{+}^{\prime}<x_{f+}$ in the form:

$$
G_{\alpha_{f} \alpha_{i}}\left(X_{f}, X_{i}, \mid p_{+}\right)=\int_{\mathbf{x}^{\prime}\left(x_{+}^{\prime}\right)} G_{\alpha_{f} \alpha^{\prime}}\left(X_{f}, X^{\prime} \mid p_{+}\right) G_{\alpha^{\prime} \alpha_{i}}\left(X^{\prime}, X_{i} \mid p_{+}\right) .
$$

\subsection{Separation into regions}

Using equation (3.1), it is possible to identify, depending on the number of Wilson lines propagating simultaneously, a single in-medium region for the out - out contribution, two regions for the $i n$ - out and three different regions for the $i n$-in term, as shown in figure 3. For the out-out term, eq. (2.10), no separation is necessary as the only configuration that propagates through the medium is that involving two Wilson lines (for the initial quark in both amplitude and complex conjugate amplitude). Omitting kinematical terms,

$$
\left\langle\left|\mathcal{T}_{\text {out }}\right|^{2}\right\rangle \propto \frac{1}{N}\left\langle\operatorname{Tr}\left(G\left(X, X_{0} \mid p_{0+}\right) G^{\dagger}\left(\bar{X}, \bar{X}_{0} \mid p_{0+}\right)\right)\right\rangle_{\left(L_{+}, x_{0+}\right)},
$$

where the longitudinal support for the average is explicitly shown as a subscript.

For the in-out contribution we identify two separate regions (see figure $3 \mathrm{~b}$ ): from the production point $x_{0+}$ to the gluon emission point $x_{1+}$, where there are just two fundamental Wilson lines, and from $x_{1+}$ to the end of the medium $L_{+}$where an additional adjoint 


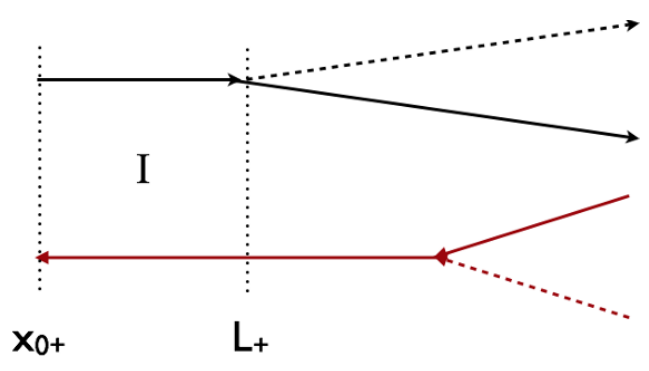

(a) Sections for $\left\langle\left|\mathcal{T}_{\text {out }}\right|^{2}\right\rangle$.

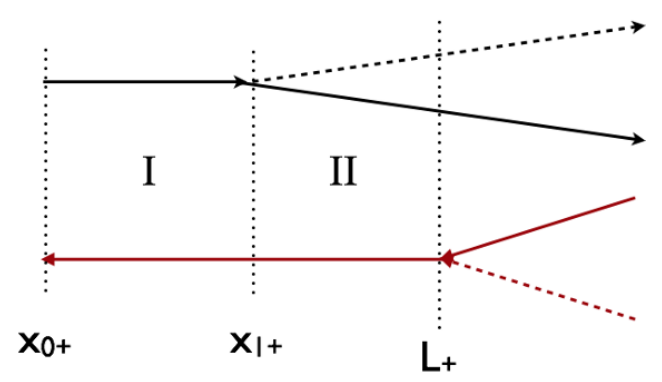

(b) Sections for $\left\langle\mathcal{T}_{\text {in }} \mathcal{T}_{\text {out }}^{\dagger}\right\rangle$.

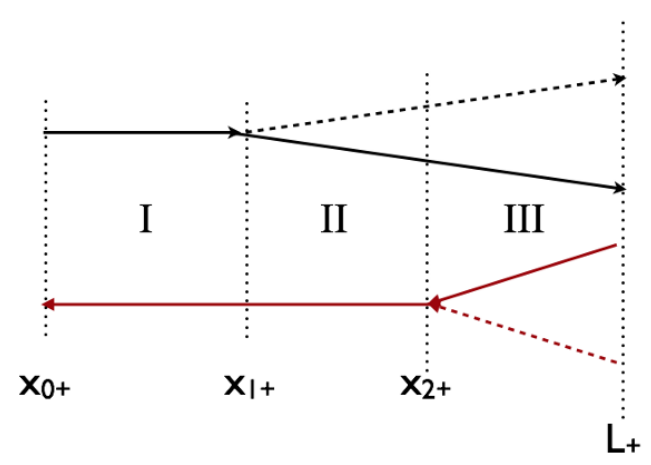

(c) Sections for $\left\langle\left|\mathcal{T}_{\text {in }}\right|^{2}\right\rangle$.

Figure 3: Schematic view of the sections division. The full arrows represent the quarks and the dashed arrows the gluons. Black arrows hold for the amplitude and red arrows for the complex conjugate amplitude.

Wilson line is present. To account for this separation, the Wilson line that comes from the complex conjugate amplitude, represented as a red arrow in figure 4, has to be separated as in equation (3.1) with the introduction of additional transverse coordinates. The new transverse coordinates are represented in red, while the remaining transverse structure in black. Explicit calculations are shown in appendix B.2, but hereon, we will restrict ourselves to the large- $N$ limit. Omitting kinematical terms, the result reads

$$
\begin{aligned}
\left\langle\mathcal{T}_{\text {in }} \mathcal{T}_{\text {out }}^{\dagger}\right\rangle \propto & \frac{1}{2 N^{2}} \int_{\overline{\mathbf{x}}_{1}} \int_{Z_{1}}^{Z} \mathcal{D} \mathbf{w}_{2}\left(\xi_{2}\right) \exp \left\{\frac{i k_{+}}{2} \int^{\xi_{2}} \dot{\mathbf{w}}_{2}\left(\xi_{2}\right)\right\} \\
& \times\left\langle\operatorname{Tr}\left(G\left(Y, Y_{1} \mid q_{+}\right) W^{\dagger}\left(\mathbf{w}_{2}\right)\right)\right\rangle\left\langle\operatorname{Tr}\left(W\left(\mathbf{w}_{2}\right) G^{\dagger}\left(\bar{X}, \bar{X}_{1} \mid p_{0+}\right)\right)\right\rangle_{\left(L_{+}, x_{1+}\right)} \\
& \times\left.\left\langle\operatorname{Tr}\left(G\left(X_{1}, X_{0} \mid p_{+}\right) G^{\dagger}\left(\bar{X}_{1}, \bar{X}_{0} \mid p_{+}\right)\right)\right\rangle_{\left(x_{1+}, x_{0+}\right)}\right|_{Z_{1}=Y_{1}=X_{1}}
\end{aligned}
$$

where $\xi_{2} \in\left[x_{1+}, L_{+}\right]$.

Proceeding analogously for the in - in term, 3 regions can be defined (figure 3c). A schematic representation of the transverse coordinate structure of this expression is provided in figure 5. The complete result is derived in appendix B. 2 but, in the large- $N$ 

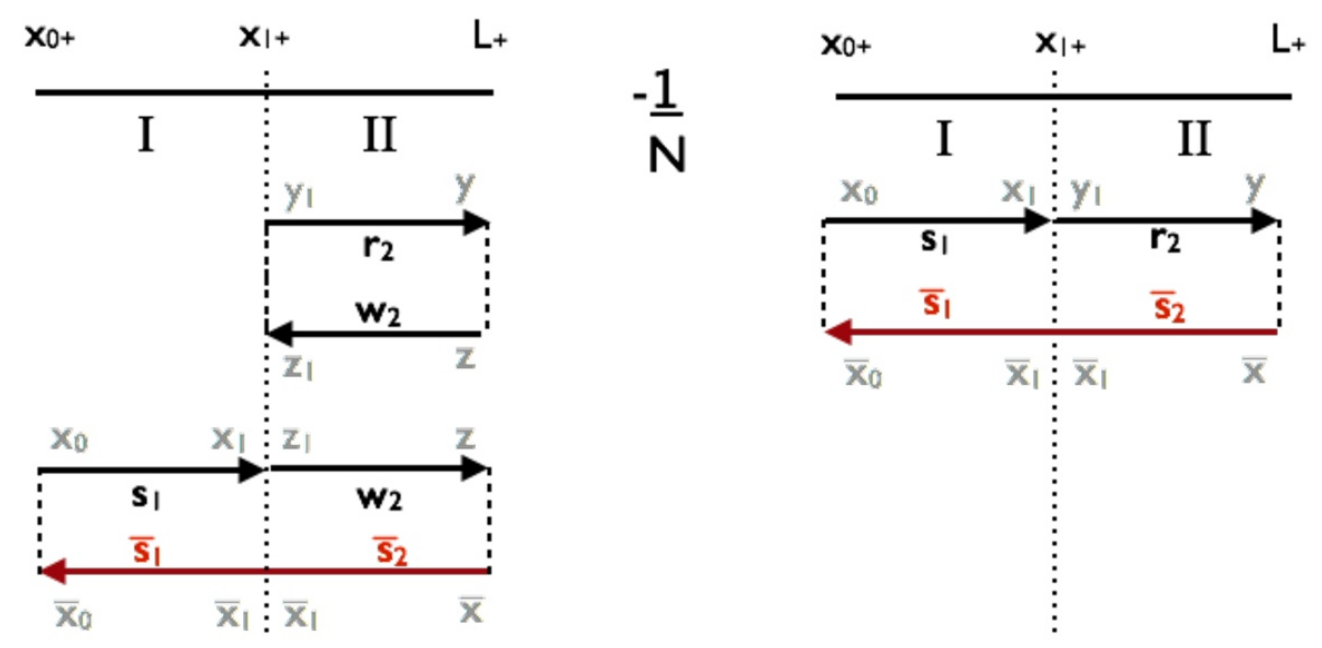

Figure 4: Schematic representation of the transverse structure for the amplitude $\mathcal{T}_{\text {in }} \mathcal{T}_{\text {out }}^{\dagger}$. The transverse ending points are written in grey, while the transverse coordinates to be integrated out in black and red. The red coordinates correspond to the propagators that were divided into different regions (note that $\mathbf{z}_{1}=\mathbf{y}_{1}=\mathbf{x}_{1}$ ).

limit, ${ }^{5}$ reads

$$
\begin{aligned}
\left\langle\left|\mathcal{T}_{\text {in }}\right|^{2}\right\rangle \propto & \int_{\overline{\mathbf{x}}_{1}, \overline{\mathbf{x}}_{2}, \mathbf{y}_{2}, \mathbf{z}_{2}} \int_{Z_{1}}^{Z_{2}} \mathcal{D} \mathbf{w}_{2}\left(\xi_{2}\right) \exp \left\{\frac{i k_{+}}{2} \int^{\xi_{2}} \dot{\mathbf{w}}_{2}\left(\xi_{2}\right)\right\} \\
& \times \int_{Z_{2}}^{Z} \mathcal{D} \mathbf{w}_{3}\left(\xi_{3}\right) \int_{\bar{Z}_{2}}^{\bar{Z}} \mathcal{D} \overline{\mathbf{w}}_{3}\left(\xi_{3}\right) \exp \left\{\frac{i k_{+}}{2} \int^{\xi_{3}}\left(\dot{\mathbf{w}}_{3}\left(\xi_{3}\right)-\dot{\overline{\mathbf{w}}}_{3}\left(\xi_{3}\right)\right)\right\} \\
& \times\left\langle\left[W\left(\mathbf{w}_{3}\right) W^{\dagger}\left(\overline{\mathbf{w}}_{3}\right)\right]_{j i}\left[W^{\dagger}\left(\mathbf{w}_{3}\right) W\left(\overline{\mathbf{w}}_{3}\right) G^{\dagger}\left(\bar{Y}, \bar{Y}_{2} \mid q_{+}\right) G\left(Y, Y_{2} \mid q_{+}\right)\right]_{l k}\right\rangle_{\left(L_{+}, x_{2+}\right)} \\
& \times\left\langle\left[G\left(Y_{2}, Y_{1} \mid q_{+}\right) W^{\dagger}\left(\mathbf{w}_{2}\right)\right]_{i j}\left[G^{\dagger}\left(\bar{X}_{2}, \bar{X}_{1} \mid p_{+}\right) W\left(\mathbf{w}_{2}\right)\right]_{k l}\right\rangle_{\left(x_{2+}, x_{1+}\right)} \\
& \times\left.\left\langle\operatorname{Tr}\left(G\left(X_{1}, X_{0} \mid p_{+}\right) G^{\dagger}\left(\bar{X}_{1}, \bar{X}_{0} \mid p_{+}\right)\right)\right\rangle_{\left(x_{1+}, x_{0+}\right)}\right|_{Z_{1}=Y_{1}=X_{1}}
\end{aligned}
$$

where $\xi_{2} \in\left[x_{1+}, x_{2+}\right]$ and $\xi_{3} \in\left[x_{2+}, L_{+}\right]$.

Focusing now only on the coloured part of equations (3.2), (3.3) and (3.4), we expand the Wilson line up to second order in the medium fields. In particular, the result for the 2-point function is (eq. (C.9))

$$
\frac{1}{N}\left\langle W(\mathbf{x}) W^{\dagger}(\mathbf{y})\right\rangle=\mathrm{e}^{-C_{F} v\left(\mathbf{s}_{1}-\overline{\mathbf{s}}_{1}\right)},
$$

with

$$
v(\mathbf{x}-\mathbf{y})=\frac{1}{2} \int d \xi_{+} \sigma(\mathbf{x}-\mathbf{y}) n\left(\xi_{+}\right),
$$

\footnotetext{
${ }^{5}$ Note that we assume $x_{1+}<x_{2+}$ here. Since the result for $x_{1+}>x_{2+}$ is the complex conjugate of the one for $x_{1+}<x_{2+}$, to get the final result we will multiply by 2 and take the real part.
} 


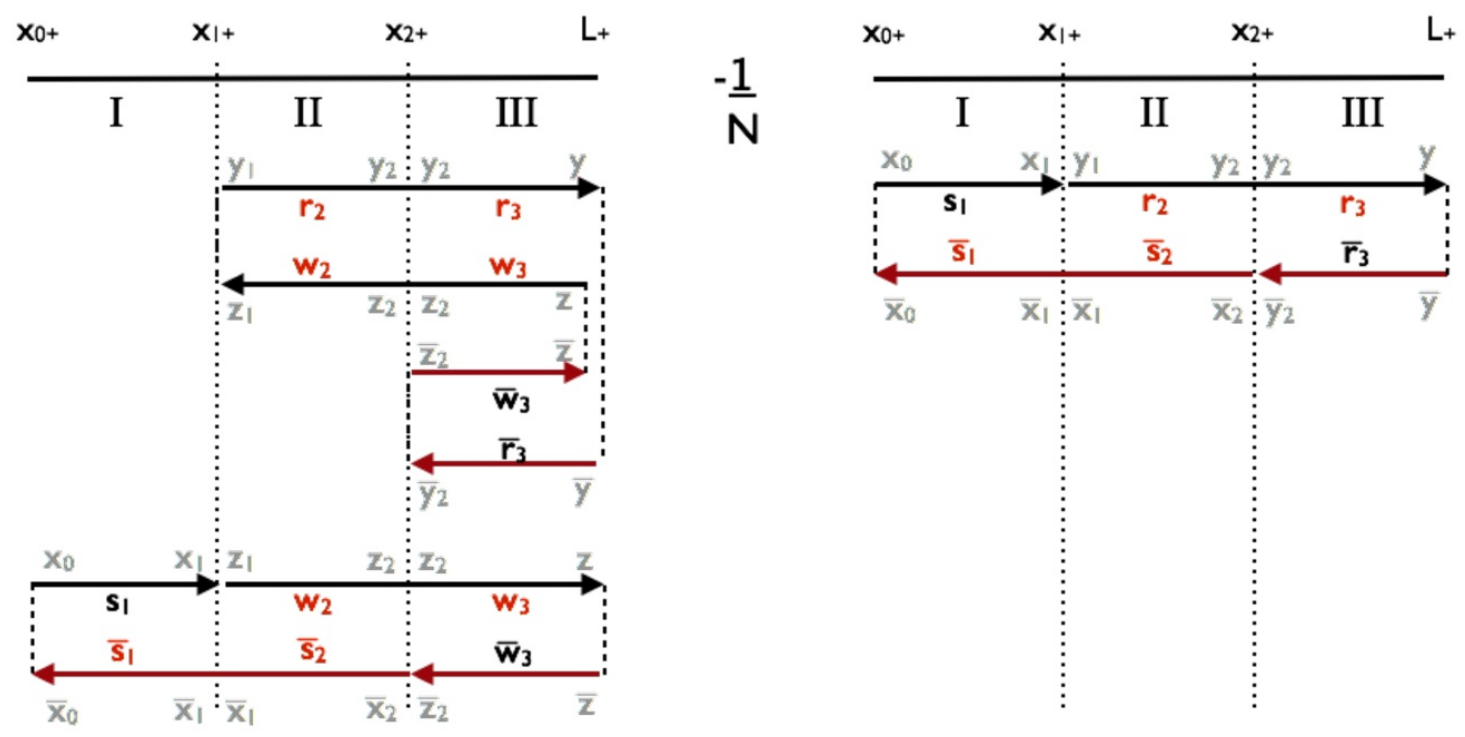

Figure 5: Schematic representation of the transverse structure for the amplitude $\left|\mathcal{T}_{\text {in }}\right|^{2}$. The transverse ending points are represented in grey, while the transverse coordinates to be integrated out in black and red. The red coordinates correspond to the propagators that were divided into different regions (note that $\mathbf{z}_{1}=\mathbf{y}_{1}=\mathbf{x}_{1}$ and $\mathbf{z}_{2}=\mathbf{y}_{2}=\mathbf{x}_{2}$ ).

where $n\left(\xi_{+}\right)$is the longitudinal density of scattering centers in the medium and $\sigma$ the cross section for scattering of the dipole formed by the particles located at transverse coordinates $\mathbf{x}$ and $\mathbf{y}$ with the medium:

$$
\sigma(\mathbf{x}-\mathbf{y})=2 g^{2} \int \frac{d \mathbf{q}}{(2 \pi)^{2}}\left|a_{-}(\mathbf{q})\right|^{2}\left(1-\mathrm{e}^{i \mathbf{q} \cdot(\mathbf{x}-\mathbf{y})}\right) .
$$

Using the results derived in appendix C.2, region II of the $i n-i n$ contribution can be factorised at large $N$ into the independent average of two dipoles formed by the final quark and gluon, while region III (see appendix C.3) into a dipole times an independent quadrupole. Thus

$$
\begin{aligned}
& \left\langle\left|\mathcal{T}_{\text {in }}\right|^{2}\right\rangle \propto \int_{\overline{\mathbf{x}}_{1}, \overline{\mathbf{x}}_{2},} \frac{1}{2 N^{3}} \int_{Z_{1}}^{Z_{2}} \mathcal{D} \mathbf{w}_{2}\left(\xi_{2}\right) \exp \left\{\frac{i k_{+}}{2} \int^{\xi_{2}} \dot{\mathbf{w}}_{2}\left(\xi_{2}\right)\right\} \\
& \times \int_{Z_{2}}^{Z} \mathcal{D} \mathbf{w}_{3}\left(\xi_{3}\right) \int_{\bar{Z}_{2}}^{\bar{Z}} \mathcal{D} \overline{\mathbf{w}}_{3}\left(\xi_{3}\right) \exp \left\{\frac{i k_{+}}{2} \int^{\xi_{3}}\left(\dot{\mathbf{w}}_{3}\left(\xi_{3}\right)-\dot{\overline{\mathbf{w}}}_{3}\left(\xi_{3}\right)\right)\right\} \\
& \times\left\langle\operatorname{Tr}\left(W^{\dagger}\left(\mathbf{w}_{3}\right) W\left(\overline{\mathbf{w}}_{3}\right) G^{\dagger}\left(\bar{Y}, \bar{Y}_{2} \mid q_{+}\right) G\left(Y, Y_{2} \mid q_{+}\right)\right)\right\rangle_{\left(L_{+}, x_{2+}\right)} \\
& \times\left\langle\operatorname{Tr}\left(W\left(\mathbf{w}_{3}\right) W^{\dagger}\left(\overline{\mathbf{w}}_{3}\right)\right)\right\rangle_{\left(x_{2+}, L_{+}\right)}\left\langle\operatorname{Tr}\left(G\left(Y_{2}, Y_{1} \mid q_{+}\right) W^{\dagger}\left(\mathbf{w}_{2}\right)\right)\right\rangle_{\left(x_{2+}, x_{1+}\right)} \\
& \times\left\langle\operatorname{Tr}\left(G^{\dagger}\left(\bar{X}_{2}, \bar{X}_{1} \mid p_{+}\right) W\left(\mathbf{w}_{2}\right)\right)\right\rangle_{\left(x_{2+}, x_{1+}\right)} \\
& \times\left\langle\operatorname{Tr}\left(G\left(X_{1}, X_{0} \mid p_{+}\right) G^{\dagger}\left(\bar{X}_{1}, \bar{X}_{0} \mid p_{+}\right)\right)\right\rangle_{\left(x_{1+}, x_{0+}\right)} \mid \begin{array}{l}
{\substack{Z_{1}=Y_{1}=X_{1} \\
\bar{Z}_{2}=\bar{Y}_{2}=\bar{X}_{2}}} \\
.
\end{array}
\end{aligned}
$$




\subsection{Dipole approximation}

For an opaque media, the dipole cross section can be approximated by its small distance component $[49,50]$,

$$
n(\xi) \sigma(\mathbf{r}) \simeq \frac{1}{2} \hat{q} \mathbf{r}^{2}+\mathcal{O}\left(\mathbf{r}^{2} \ln \mathbf{r}^{2}\right)
$$

where $\hat{q}$, the transport coefficient, characterises the typical squared transverse momentum that the particle acquires, per mean free path $\lambda$, from the interaction with the medium. This approximation, alternatively referred to as multiple soft scattering approximation or dipole approximation, is valid for small transverse distances $\mathbf{r}$. Although the medium is expanding, we will perform, for simplicity, the calculations for an homogeneous static medium for which $\hat{q}$ is a constant. ${ }^{6}$

The result for the 4-point correlation function is explicitly derived in appendix C.2 in the Gaussian approximation for field correlators. For large $N$ and in region III, equation (C.30) can be written (see the notation in appendices C.1 and C.2, and figure 5 for the coordinates) as

$$
\begin{aligned}
& \left\langle\operatorname{Tr}\left(W^{\dagger}\left(\mathbf{w}_{3}\right) W\left(\overline{\mathbf{w}}_{3}\right) W^{\dagger}\left(\overline{\mathbf{r}}_{3}\right) W\left(\mathbf{r}_{3}\right)\right)\right\rangle_{\left(L_{+}, x_{2+}\right)} \\
& \quad=\frac{1}{N}\left\langle\operatorname{Tr}\left(W\left(\overline{\mathbf{w}}_{3}\right) W^{\dagger}\left(\mathbf{w}_{3}\right)\right)\right\rangle_{\left(L_{+}, x_{2+}\right)}\left\langle\operatorname{Tr}\left(W\left(\mathbf{r}_{3}\right) W^{\dagger}\left(\overline{\mathbf{r}}_{3}\right)\right)\right\rangle_{\left(L_{+}, x_{2}+\right)} \Delta_{\mathrm{coh}},
\end{aligned}
$$

with the colour decoherence parameter given by

$$
\begin{aligned}
\Delta_{\mathrm{coh}} & =1+\left.\int_{x_{2+}}^{L_{+}} d \tau N m_{12}(\tau) e^{N\left(m_{11}-m_{22}\right)}\right|_{\left(\tau, x_{2+}\right)}, \\
m_{11}-m_{22} & =\frac{1}{2}\left[v\left(\mathbf{r}_{3}-\overline{\mathbf{r}}_{3}\right)+v\left(\mathbf{w}_{3}-\overline{\mathbf{w}}_{3}\right)-v\left(\mathbf{r}_{3}-\mathbf{w}_{3}\right)-v\left(\overline{\mathbf{w}}_{3}-\overline{\mathbf{r}}_{3}\right)\right] \\
& \simeq-\frac{\hat{q}}{2} \int_{\tau}^{x_{2+}} d \xi\left(\mathbf{r}_{3}-\overline{\mathbf{w}}_{3}\right) \cdot\left(\overline{\mathbf{r}}_{3}-\mathbf{w}_{3}\right), \\
m_{12}(\tau) & =\frac{1}{2}\left[v\left(\mathbf{r}_{3}-\overline{\mathbf{w}}_{3}\right)+v\left(\mathbf{w}_{3}-\overline{\mathbf{r}}_{3}\right)-v\left(\mathbf{r}_{3}-\mathbf{w}_{3}\right)-v\left(\overline{\mathbf{r}}_{3}-\overline{\mathbf{w}}_{3}\right)\right](\tau) \\
& \simeq \frac{\hat{q}}{2}\left(\mathbf{r}_{3}-\overline{\mathbf{r}}_{3}\right) \cdot\left(\mathbf{w}_{3}-\overline{\mathbf{w}}_{3}\right),
\end{aligned}
$$

where $\simeq$ in (3.12) and (3.13) holds in the dipole approximation.

With this factorisation (3.10), a friendly interpretation of the colour structure for every contribution to the total production cross section can be obtained, see figure 6 . Region I corresponds to the Brownian motion of the initial quark before it emits. In region II, corresponding to the gluon formation time $\tau_{\text {form }}$, the final quark and gluon are colour correlated - this is easy to see as the spectrum is proportional to two traces of Wilson lines in this region. ${ }^{7}$ Region III, on the other hand, admits a nice interpretation as the independent broadening of the quark and the produced gluon times a decoherence parameter controlling the probability that the gluon decorrelates in colour from the quark. Only in this case of decorrelation the spectrum exists, the opposite possibility being suppressed.

\footnotetext{
${ }^{6}$ An expanding medium can be accounted for by a change of variables [38, 51, 52].

${ }^{7}$ Remember that the trace of two Wilson lines is proportional to the $S$-matrix of the process, rather than to the scattering amplitude, hence to the probability of no interaction - this is a standard result, see e.g. [38].
} 


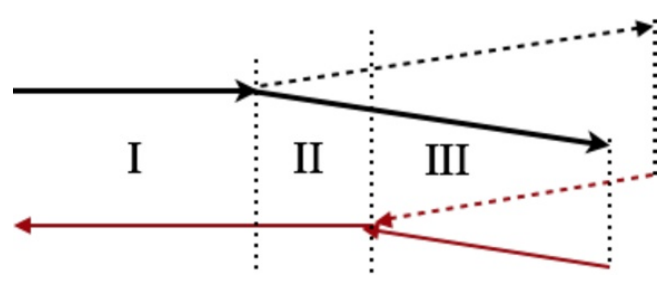

(a) Final configuration in which the gluon and the quark evolve independently.

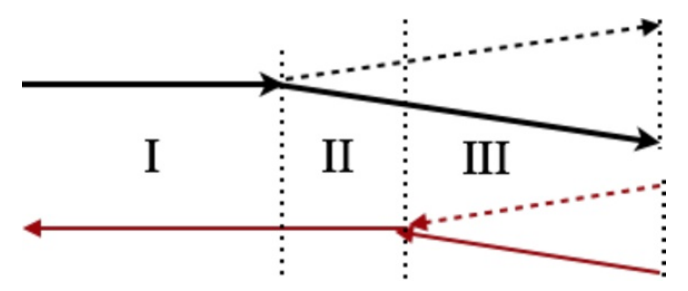

(b) Final configuration in which the gluon and the quark remain correlated.

Figure 6: Final particle configurations for $\left\langle\left|\mathcal{T}_{\text {in }}\right|^{2}\right\rangle$.

Let us elaborate more on this important point now. The $\Delta_{\text {coh }}$ parameter defined in (3.11) that controls the decoherence of colour sources contains the same physics of colour decoherence as the the one, named $\Delta_{\text {med }}$, previously found in the antenna radiation [ 40 , $41,43,44] .{ }^{8}$ Note that the exponent in $\Delta_{\text {coh }}$ given in (3.12) is proportional to the distance between the quark and the gluon. The medium-induced spectrum is suppressed for the case $\Delta_{\text {coh }} \rightarrow 0$, that is when the quark and the gluon remain in a colour coherent state. In the opposite limit, $\Delta_{\text {coh }} \rightarrow 1$, the quark and the gluon lose their coherence and appear as two independent particles. In this sense, a medium-induced radiation in which the quark and the gluon remain in a colour coherent state is exponentially suppressed. For the particular case in which $x_{2+} \ll L_{+}$a suppression factor of the form $\tau_{\text {form }} / L_{+}$appears as shown previously in $[37]^{9}$. Equation (3.11) shows clearly that this approximation deteriorates more and more when the position of the splitting vertex gets closer and closer to the end of the medium.

One additional comment is in order: both in the BDMPS limit with strictly eikonal quark lines $\left(\mathbf{r}_{3}=\overline{\mathbf{r}}_{3}\right)$ or in the limit of hard gluon emissions [36] with strictly eikonal gluon lines $\left(\mathbf{w}_{3}=\overline{\mathbf{w}}_{3}\right), \Delta_{\text {coh }}=1$ and the independent broadening of the $q$ and $g$, given by the traces in (3.10), happens instantaneously with no colour interference between quark and gluon in region III.

\section{Gluon emission spectrum}

\subsection{Vacuum gluon spectrum}

In the previous section, all possible colour medium configurations were taken into account by averaging over the whole ensemble of possible colour profiles. The results -

\footnotetext{
${ }^{8}$ Indeed, our $\Delta_{\text {coh }}$ would provide corrections to the prefactor in the direct contributions to the antenna spectrum.

${ }^{9}$ This can be seen in the following way: the coherent piece, called non-factorisable in [37], can be written

$$
\left.\left.\int_{x_{2+}}^{L_{+}} d \tau e^{N m_{22}}\right|_{\left(L_{+}, \tau\right)} N m_{12}(\tau) e^{N m_{11}}\right|_{\left(\tau, x_{2+}\right)}
$$

with the exponential in $m_{22}$ (in $m_{11}$ ) giving the independent broadening of quark and gluon (the colour coherence propagation of the quark-gluon system). The latter suppresses exponentially $\tau>x_{2+}+\tau_{\text {form }}$, while $m_{12}$ can be expressed as the product of gradients of the former. Such product of gradients is proportional to $1 /\left(L_{+}-\tau\right)$.
} 


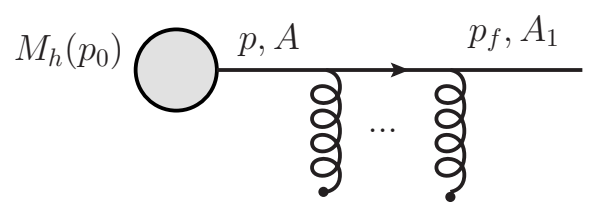

Figure 7: Diagram representing the elastic propagation of a quark through a coloured medium.

eqs. (3.2), (3.3) and (3.8) - are for some general trajectories in the transverse plane, $\mathbf{r}=\mathbf{r}(\xi)$, that change with the propagation time $\xi$. In order to account for the transverse broadening of the propagating particles, it is necessary to integrate over all possible trajectories that each particle may undergo. Particular examples are computed in appendix D using a semi-classical approximation. This method, that is able to provide an exact solution for some cases (see appendix D.1), assumes that the dominant contribution to the path integral is mainly given by the classical trajectory plus local fluctuations at the end points of the trajectory. The classical path is determined taking into account the kinetic term from the propagator $G_{0}$, eq. (2.3), and the potential term from the effective action of the medium scattering centres encoded in the several $n$-point correlation function.

Using these results from appendix D.1, in particular (D.28), and after Fourier transforming from coordinate space to momentum space, the out - out term can be finally written as

$$
\begin{aligned}
\left\langle\left|\mathcal{T}_{\text {out }}\right|^{2}\right\rangle= & g^{2} \frac{4 \sqrt{2} \zeta(1-\zeta) p_{0+}}{[(1-\zeta) \mathbf{k}-\zeta \mathbf{q}]^{2}} P_{g \leftarrow q}(\zeta) \mathcal{P}\left(\mathbf{p}_{0} \rightarrow \mathbf{k}+\mathbf{q}\right) \\
& \times(2 \pi)^{4} \delta\left(k+q-p_{0}\right)_{+}^{2}\left|M_{h}\left(p_{0+}\right)\right|^{2} \int_{\mathbf{b}},
\end{aligned}
$$

where the integral over $\mathbf{b}$ comes from the fact that we are using plane waves, and

$$
\mathcal{P}\left(\mathbf{p}_{0} \rightarrow \mathbf{p}_{f}\right)=\frac{1}{\pi \Delta \xi_{1} \hat{q}_{F}} \exp \left\{-\frac{\left(\mathbf{p}_{f}-\mathbf{p}_{0}\right)^{2}}{\Delta \xi_{1} \hat{q}_{F}}\right\},
$$

with $\hat{q}_{F}=C_{F} \hat{q}$ and $\Delta \xi_{1}=L_{+}-x_{0+}$. This expression, normalised to one, simply provides the momentum broadening of the initial quark that propagates through a medium.

The corresponding number of emitted gluons is

$$
\frac{d^{2} I_{\text {out }}}{d \Omega_{q} d \Omega_{k}}=\frac{1}{\sigma_{\text {el }}} \frac{d^{2} \sigma_{\text {out }}}{d \Omega_{q} d \Omega_{k}}=\frac{\left\langle\left|\mathcal{T}_{\text {out }}\right|^{2}\right\rangle}{\sigma_{\mathrm{el}}},
$$

where $\sigma_{\mathrm{el}}$ is the total elastic cross section. Using the same assumptions as in section 2.2, it is possible to calculate the elastic channel that is schematically represented in figure 7 . The result reads

$$
\sigma_{\mathrm{el}}=\int d \Omega_{p} \frac{d I_{\mathrm{el}}}{d \Omega_{p}}=\sqrt{2}\left|M_{h}\left(p_{0+}\right)\right|^{2}(2 \pi) \delta(0) \int_{\mathbf{b}} .
$$

Finally, one finds that

$$
\frac{d^{2} I_{\text {out }}}{d \Omega_{q} d \Omega_{k}}=g^{2}(2 \pi)^{3} \frac{4 \zeta(1-\zeta) p_{0+}}{[(1-\zeta) \mathbf{k}-\zeta \mathbf{q}]^{2}} P_{g \leftarrow q}(\zeta) \mathcal{P}\left(\mathbf{p}_{0} \rightarrow \mathbf{k}+\mathbf{q}\right) \delta\left(k+q-p_{0}\right)_{+},
$$

where all undetermined factors cancel. 
Eq. (4.5) admits a simple interpretation as the typical vacuum emission for an off-shell quark which, however, has experienced broadening while traversing the medium. This fact can be more clearly seen by taking the limit $\hat{q}_{F} L_{+} \rightarrow 0$ in which the vacuum spectrum is recovered. In this limit, the integration over the quark phase space can be performed, and fixing the initial transverse direction $\mathbf{p}_{0}=\mathbf{0}$ one indeed obtains the expected expression with the corresponding Altarelli-Parisi splitting function ${ }^{10}$

$$
\left.\frac{d^{2} I_{\mathrm{out}}}{d \zeta d \mathbf{k}}\right|_{\hat{q}_{F} L_{+} \rightarrow 0}=\frac{d^{2} I_{\mathrm{vac}}}{d \zeta d \mathbf{k}}=\frac{\alpha_{s}}{2 \pi^{2}} \frac{1}{\mathbf{k}^{2}} P_{g \leftarrow q}(\zeta) .
$$

\subsection{Medium emission spectrum}

The remaining two terms (in - in and in - out) can be identified with the medium contribution. ${ }^{11}$ The result reads

$$
\begin{aligned}
\frac{d^{2} I_{\text {med }}}{d \Omega_{q} d \Omega_{k}}= & \frac{\left\langle\left|\mathcal{T}_{\text {med }}\right|^{2}\right\rangle}{\sigma_{\mathrm{el}}}=\frac{\left\langle\left|\mathcal{T}_{\text {in }}\right|^{2}\right\rangle+2 \operatorname{Re}\left\langle\mathcal{T}_{\text {in }} \mathcal{T}_{\text {out }}^{\dagger}\right\rangle}{\sigma_{\mathrm{el}}} \\
= & 2 g^{2} P_{g \leftarrow q}(\zeta) \operatorname{Re} \int_{\mathbf{x}_{0}, \mathbf{x}_{1}, \mathbf{y}, \mathbf{z}, \overline{\mathbf{x}}_{0}, \overline{\mathbf{x}}_{1}}^{x_{1+}} \mathrm{e}^{i \mathbf{p}_{0} \cdot\left(\mathbf{x}_{0}-\overline{\mathbf{x}}_{0}\right)} \Sigma_{1}\left(x_{0+}, x_{1+}, \mathbf{x}_{0}, \overline{\mathbf{x}}_{0}, \mathbf{x}_{1}, \overline{\mathbf{x}}_{1}\right) \\
& \times\left\{\frac{1}{\zeta(1-\zeta) p_{0+}} \int_{\mathbf{y}_{2}, \mathbf{z}_{2}, \overline{\mathbf{x}}_{2}, \overline{\mathbf{y}}, \overline{\mathbf{z}}}^{x_{2+}} \mathrm{e}^{-i \mathbf{k} \cdot(\mathbf{z}-\overline{\mathbf{z}})-i \mathbf{q} \cdot(\mathbf{y}-\overline{\mathbf{y}})}\right. \\
& \times\left[\left((1-\zeta) \frac{\partial}{\partial \mathbf{z}_{1}}-\zeta \frac{\partial}{\partial \mathbf{y}_{1}}\right) \cdot\left((1-\zeta) \frac{\partial}{\partial \overline{\mathbf{z}}_{2}}-\zeta \frac{\partial}{\partial \overline{\mathbf{y}}_{2}}\right)\right] \\
& \times \Sigma_{2}\left(x_{1+}, x_{2+}, \mathbf{y}_{1}, \mathbf{z}_{1}, \overline{\mathbf{x}}_{1}, \mathbf{y}_{2}, \mathbf{z}_{2}, \overline{\mathbf{x}}_{2}\right) \Sigma_{3}\left(x_{2+}, L_{+}, \mathbf{y}_{2}, \mathbf{z}_{2}, \overline{\mathbf{y}}_{2}, \overline{\mathbf{z}}_{2}, \mathbf{y}, \mathbf{z}, \overline{\mathbf{y}}, \overline{\mathbf{z}}\right) \\
& +2 \int_{\overline{\mathbf{x}}} \mathrm{e}^{-i \mathbf{k} \cdot(\mathbf{z}-\overline{\mathbf{x}})-i \mathbf{q} \cdot(\mathbf{y}-\overline{\mathbf{x}})} \\
& \times \frac{(1-\zeta) \mathbf{k}-\zeta \mathbf{q}}{((1-\zeta) \mathbf{k}-\zeta \mathbf{q})^{2}} \cdot\left((1-\zeta) \frac{\partial}{\partial \mathbf{z}_{1}}-\zeta \frac{\partial}{\partial \mathbf{y}_{1}}\right) \\
& \left.\times \Sigma_{2}\left(x_{1+}, L_{+}, \mathbf{y}_{1}, \mathbf{z}_{1}, \overline{\mathbf{x}}_{1}, \mathbf{y}, \mathbf{z}, \overline{\mathbf{x}}\right)\right\}\left.\left[\delta^{(2)}(0)\right]^{-1} \delta\left(k+q-p_{0}\right)_{+}\right|_{\substack{\mathbf{z}_{1}=\mathbf{y}_{1}=\mathbf{x}_{1} \\
\mathbf{z}_{2}=\mathbf{y}_{2}=\overline{\mathbf{x}}_{2}}},
\end{aligned}
$$

where $\Sigma_{i}$ are the results of the path integrals for each region $i=\mathrm{I}$, II, III (see appendix D for definitions, in particular eqs. (D.28), (D.37) and (D.43)). Note that the factor $C_{F}$ (explicit in the $\left\langle\mathcal{T}_{\text {in }} \mathcal{T}_{\text {out }}^{\dagger}\right\rangle$ contribution) should be approximated by its large- $N$ limit, $N / 2$.

Performing the integration over $\mathbf{x}_{0}$ and $\overline{\mathbf{x}}_{0}, \Sigma_{1}$ can be simplified and eq. (4.7) results in

$$
\frac{d^{2} I_{\text {med }}}{d \Omega_{q} d \Omega_{k}}=\frac{\left\langle\left|\mathcal{T}_{\text {med }}\right|^{2}\right\rangle}{\sigma_{\text {el }}}=\frac{\left\langle\left|\mathcal{T}_{\text {in }}\right|^{2}\right\rangle+2 \operatorname{Re}\left\langle\mathcal{T}_{\text {in }} \mathcal{T}_{\text {out }}^{\dagger}\right\rangle}{\sigma_{\text {el }}}
$$

\footnotetext{
${ }^{10}$ In [36] we were only able to recover the splitting function in the considered limit, $P_{g \leftarrow q}(\xi \rightarrow 1)$.

${ }^{11}$ The medium spectra in [36] is recovered doing $\mathbf{k}=-\mathbf{q}$, and $p_{0+}=q_{+}$or $p_{0+}=k_{+}$.
} 


$$
\begin{aligned}
= & 2 g^{2} P_{g \leftarrow q}(\zeta) \operatorname{Re} \int_{\mathbf{x}_{1}, \mathbf{y}, \mathbf{z}, \overline{\mathbf{x}}_{1}}^{x_{1+}} \mathrm{e}^{i \mathbf{p}_{0} \cdot\left(\mathbf{x}_{1}-\overline{\mathbf{x}}_{1}\right)} \exp \left\{-\frac{\hat{q}_{F} \Delta t_{1}}{4}\left(\mathbf{x}_{1}-\overline{\mathbf{x}}_{1}\right)^{2}\right\} \\
& \times\left\{\frac{1}{\zeta(1-\zeta) p_{0+}} \int_{\mathbf{y}_{2}, \mathbf{z}_{2}, \overline{\mathbf{x}}_{2}, \overline{\mathbf{y}}, \overline{\mathbf{z}}}^{x_{2+}} \mathrm{e}^{-i \mathbf{k} \cdot(\mathbf{z}-\overline{\mathbf{z}})-i \mathbf{q} \cdot(\mathbf{y}-\overline{\mathbf{y}})}\right. \\
& \times\left[\left((1-\zeta) \frac{\partial}{\partial \mathbf{z}_{1}}-\zeta \frac{\partial}{\partial \mathbf{y}_{1}}\right) \cdot\left((1-\zeta) \frac{\partial}{\partial \overline{\mathbf{z}}_{2}}-\zeta \frac{\partial}{\partial \overline{\mathbf{y}}_{2}}\right)\right] \\
& \times \Sigma_{2}\left(x_{1+}, x_{2+}, \mathbf{y}_{1}, \mathbf{z}_{1}, \overline{\mathbf{x}}_{1}, \mathbf{y}_{2}, \mathbf{z}_{2}, \overline{\mathbf{x}}_{2}\right) \Sigma_{3}\left(x_{2+}, L_{+}, \mathbf{y}_{2}, \mathbf{z}_{2}, \overline{\mathbf{y}}_{2}, \overline{\mathbf{z}}_{2}, \mathbf{y}, \mathbf{z}, \overline{\mathbf{y}}, \overline{\mathbf{z}}\right) \\
& +2 \int_{\overline{\mathbf{x}}} \mathrm{e}^{-i \mathbf{k} \cdot(\mathbf{z}-\overline{\mathbf{x}})-i \mathbf{q} \cdot(\mathbf{y}-\overline{\mathbf{x}})} \\
& \times \frac{(1-\zeta) \mathbf{k}-\zeta \mathbf{q}}{((1-\zeta) \mathbf{k}-\zeta \mathbf{q})^{2}} \cdot\left((1-\zeta) \frac{\partial}{\partial \mathbf{z}_{1}}-\zeta \frac{\partial}{\partial \mathbf{y}_{1}}\right) \\
& \left.\times \Sigma_{2}\left(x_{1+}, L_{+}, \mathbf{y}_{1}, \mathbf{z}_{1}, \overline{\mathbf{x}}_{1}, \mathbf{y}, \mathbf{z}, \overline{\mathbf{x}}\right)\right\}\left[\delta^{(2)}(0)\right]^{-1} \delta\left(k+q-p_{0}\right)_{+} \mid \begin{array}{l}
\mathbf{z}_{1}=\mathbf{y}_{1}=\mathbf{x}_{1} \\
\overline{\mathbf{z}}_{2}=\overline{\mathbf{y}}_{2}=\overline{\mathbf{x}}_{2}
\end{array}
\end{aligned}
$$

In the limit $\zeta \rightarrow 0$ and $p_{0+} \rightarrow \infty$, we recover the BDMPS-Z spectrum [53-55], see appendix E.

\section{Conclusions}

In this work, we provide a complete calculation of the medium-induced single gluon radiation off a quark, in the regime where partons undergo multiple soft scatterings with the medium. The kinematic setup was extended beyond the eikonal limit by associating Brownian perturbations in the transverse plane to all propagating particles, thus extending our previous results [36] obtained in the limit of hard emitted gluons. We consider multiple scatterings of the rescattered partons and not only single scatterings as in [35]. We go beyond the work [37] by considering a finite size medium and, thus, interference effects with the vacuum radiation. This allowed us to recover the vacuum gluon radiation spectrum, with a complete factorisation of the corresponding splitting function. Our computation includes all finite-energy corrections that exist in the small angle approximation (i.e. the emission and deflections angles are small) and for static scattering centres (i.e. we do not consider recoil). We also provide technical details of the computation of the relevant $n$-point functions in colour space in the Gaussian approximation, and of the required path integrals in transverse space.

The final results, that include the evaluation of several $n$-point correlation functions and resolution up to four path-integrals, are presented in the large- $N$ limit and using the Gaussian approximation for field correlators, but without constraints on the gluon formation time. The resulting spectrum is, therefore, a generalisation of previous works, describing the radiation spectrum off a non-eikonal quark.

We confirm the finding in [37] that parton branching, in the limit of a very opaque medium, can be understood as a factorisation of single gluon emissions, where the total radiation spectrum is just an incoherent sum of each independent emitter but suppressed by the interferences with the vacuum radiation — as already derived in previous calculations 
of soft gluon emissions. As the parton shower continues its development, the medium starts to become less opaque and coherence effects between the final particles, fully included in our calculation, become important. In this case, the emission spectrum is additionally suppressed with respect to the factorised regime. This suppression is controlled by a noneikonal decoherence parameter, $\Delta_{\text {coh }}$, that accounts for the broadening of the particles and contains the same physics of colour decoherence as in the previous results in the antenna [40-44]. This fact implies that most of the energy lost by a parton must occur earlier in its development than under the assumption that the fully factorised regime holds during the full development of the shower.

\section{Acknowledgments}

We thank Tolga Altinoluk, Guillaume Beuf, Jorge Casalderrey, Fabio Domínguez, Alex Kovner, Yacine Mehtar-Tani, Cibrán Santamarina, Konrad Tywoniuk and Ricardo Vázquez for their valuable comments and useful discussions. LA, NA and CAS thank the Theory Unit at CERN for hospitality and support during stays when part of this work was developed. The work is supported by the European Research Council grant HotLHC ERC-2011StG-279579; by Ministerio de Ciencia e Innovación of Spain under projects FPA2011-22776 (LA and NA); by Xunta de Galicia (Consellería de Educación and Consellería de Innovación e Industria - Programa Incite) (LA, NA and CAS); by the Spanish Consolider-Ingenio 2010 Programme CPAN and by FEDER (LA, NA and CAS); and by Fundação para a Ciência e a Tecnologia of Portugal under project CERN/FP/123596/2011 (LA and JGM) and contracts "Investigador FCT - Development Grant" (JGM) and SFRH/BD/64543/2009 (LA).

\section{A Dirac algebra}

In order to perform the Dirac algebra in equations (2.10), (2.11) and (2.12), we recall the completeness relation for Dirac spinors,

$$
\sum_{\text {spin }} u(q, s)_{\alpha} \bar{u}(q, s)_{\beta}=q_{\alpha \beta}+m_{\alpha \beta},
$$

where the massless case will be considered. Furthermore, in the light-cone gauge $A_{+}=0$, the gluon polarizations sum to

$$
\sum_{\lambda} \epsilon_{\mu}^{*}(k, \lambda) \epsilon_{\nu}(k, \lambda)=-g_{\mu \nu}+\frac{k_{\mu} \eta_{\nu}+k_{\nu} \eta_{\mu}}{k \cdot \eta}
$$

where $\eta=(0,1, \mathbf{0})$. At this point we make the kinematics explicit:

$$
\begin{aligned}
& k_{(i)}=\left(\zeta p_{0+}, \frac{\mathbf{k}_{(i)}^{2}}{2 \zeta p_{0+}}, \mathbf{k}_{(i)}\right) \\
& q_{(i)}=\left((1-\zeta) p_{0+}, \frac{\mathbf{q}_{(i)}^{2}}{2(1-\zeta) p_{0+}}, \mathbf{q}_{(i)}\right),
\end{aligned}
$$


$i=1,2$, where $\zeta$ is the fraction of longitudinal momenta carried by the final gluon, $\mathbf{k}$ its transverse momentum and $\mathbf{q}$ the transverse momentum of the final quark. The subindex $1(2)$ correspond to the internal momenta of the gluon and quark in the amplitude (complex conjugate amplitude) when leaving the emission vertex. See eq. (2.13) and the discussion above it.

Using (A.1), (A.2) and (A.3), the Dirac structure of the out-out term can be written as

$$
\begin{gathered}
\frac{1}{8(k \cdot q)^{2}} \sum_{\text {spin, pol }} \bar{u}(q) \notin_{k}^{*}(\not k+\not q) \gamma_{+} \gamma_{0} \gamma_{+}(\not k+\not q) \notin_{k} u(q) \\
=\frac{4 \sqrt{2} \zeta(1-\zeta)}{[(1-\zeta) \mathbf{k}-\zeta \mathbf{q}]^{2}} \frac{1+(1-\zeta)^{2}}{\zeta} p_{0+},
\end{gathered}
$$

that of the in - out term as

$$
\begin{aligned}
\frac{1}{4(k \cdot q)} & \sum_{\text {spin,pol }} \bar{u}(q) \gamma_{+} \not q_{1} \notin_{k_{1}}^{*} \not p_{1} \gamma_{+} \gamma_{0} \gamma_{+}(\not k+\not q) \notin_{k} u(q) \\
& =8 \sqrt{2}(1-\zeta) p_{0+}^{2} \frac{1+(1-\zeta)^{2}}{\zeta} \frac{(1-\zeta) \mathbf{k}-\zeta \mathbf{q}}{[(1-\zeta) \mathbf{k}-\zeta \mathbf{q}]^{2}} \cdot\left((1-\zeta) \mathbf{k}_{1}-\zeta \mathbf{q}_{1}\right)
\end{aligned}
$$

and the in - in case as

$$
\begin{aligned}
& \frac{1}{2} \sum_{\text {spin,pol }} \bar{u}(q) \gamma_{+} \not \phi_{1} \notin_{k_{1}}^{*} \not p_{1} \gamma_{+} \gamma_{0} \gamma_{+} \not p_{2} \notin_{k_{2}} \phi_{2} \gamma_{+} u(q) \\
& \quad=16 \sqrt{2} \frac{1-\zeta}{\zeta} p_{0+}^{3} \frac{1+(1-\zeta)^{2}}{\zeta}\left(\left(\zeta \mathbf{q}_{1}-(1-\zeta) \mathbf{k}_{1}\right) \cdot\left(\zeta \mathbf{q}_{2}-(1-\zeta) \mathbf{k}_{2}\right)\right) .
\end{aligned}
$$

The origin of the kinematical combination $\left(\zeta \mathbf{q}_{i}-(1-\zeta) \mathbf{k}_{i}\right)$ seen in the above equation is the following: it is always possible to find a reference frame where the outgoing transverse momenta are opposite. For that, we consider a rotation operation around the $x$ axis and three generic vectors for massless partons, in the original frame, given by:

$$
p=\left(E, 0, p_{y}, p_{z}\right), \quad k=\left(\zeta E, k_{x}, k_{y}, k_{z}\right), \quad q=\left((1-\zeta) E, q_{x}, q_{y}, q_{z}\right),
$$

with $p=k+q$. The corresponding rotation matrix reads

$$
R(\theta)=\left(\begin{array}{ccc}
1 & 0 & 0 \\
0 & \cos \theta & -\sin \theta \\
0 & \sin \theta & \cos \theta
\end{array}\right) \simeq\left(\begin{array}{ccc}
1 & 0 & 0 \\
0 & 1 & -p_{\perp} / E \\
0 & p_{\perp} / E & 1
\end{array}\right)
$$

where the last equality comes from considering the eikonal approximation, $p_{\perp}, k_{\perp}, q_{\perp} \ll E$, with $p_{\perp}^{2}=p_{x}^{2}+p_{y}^{2}$ and the same for $k_{\perp}$ and $q_{\perp}\left(|\mathbf{p}|=p_{\perp}\right)$. Therefore $p_{z}=E, k_{z}=\zeta E$ and $q_{z}=(1-\zeta) E$. Designating $\overrightarrow{p^{\prime}}, \overrightarrow{k^{\prime}}$ and $\overrightarrow{q^{\prime}}$ the 3 -vectors in the rotated reference frame, one gets

$$
\overrightarrow{p^{\prime}}=R(\theta) \vec{p} \simeq\left(\begin{array}{c}
p_{x} \\
p_{y}-p_{\perp} \\
E
\end{array}\right) .
$$


Choosing the reference frame where $\mathbf{p}^{\prime}=0$,

$$
\overrightarrow{k^{\prime}} \simeq\left(\begin{array}{c}
k_{x} \\
k_{y}-\zeta p_{\perp} \\
\zeta E
\end{array}\right), \quad \overrightarrow{q^{\prime}} \simeq\left(\begin{array}{c}
q_{x} \\
q_{y}-(1-\zeta) p_{\perp} \\
(1-\zeta) E
\end{array}\right)
$$

and therefore $\mathbf{k}^{\prime}=(1-\zeta) \mathbf{k}-\zeta \mathbf{q}=-\mathbf{q}^{\prime}$.

\section{B Colour algebra}

\section{B.1 Before region separation}

To perform the colour algebra of equations (2.10), (2.11) and (2.12), we explicitly separate the path integral part of the propagators from its Wilson line part (i.e. the transverse Brownian motion from the colour rotation):

$$
\begin{aligned}
G_{\alpha_{f} \alpha_{i}}\left(X_{f}, X_{i} \mid p_{+}\right) & =\int_{X_{i}}^{X_{f}} \mathcal{D} \mathbf{r} \exp \left\{\frac{i p_{+}}{2} \int^{\xi}\left(\frac{d \mathbf{r}}{d \xi}\right)^{2}\right\} W_{\alpha_{f} \alpha_{i}}(\mathbf{r}) \\
& \equiv \mathcal{B}\left(X_{f}, X_{i} ; \mathbf{r} \mid p_{+}\right) W_{\alpha_{f} \alpha_{i}}(\mathbf{r})
\end{aligned}
$$

rewrite the adjoint Wilson lines in terms of fundamental ones (see e.g. [56]) via

$$
W_{a b}(\mathbf{x})=2 \operatorname{Tr}\left[T^{a} W^{F}(\mathbf{x}) T^{b} W^{F \dagger}(\mathbf{x})\right]
$$

and make use of the Fierz identity

$$
T_{i j}^{a} T_{k l}^{a}=\frac{1}{2}\left(\delta_{i l} \delta_{j k}-\frac{1}{N} \delta_{i j} \delta_{k l}\right)
$$

Using these expressions, we get that the colour contribution to the out-out piece reads

$$
\begin{aligned}
\left|T_{\text {out }}\right|^{2} & \propto \frac{1}{N} \operatorname{Tr}\left(T^{a} G\left(X, X_{0} \mid p_{0+}\right) G^{\dagger}\left(\bar{X}, \bar{X}_{0} \mid p_{0+}\right) T^{a}\right) \\
& =\frac{C_{F}}{N} \operatorname{Tr}\left(G\left(X, X_{0} \mid p_{0+}\right) G^{\dagger}\left(\bar{X}, \bar{X}_{0} \mid p_{0+}\right)\right)
\end{aligned}
$$

that the in - out one reads

$$
\begin{aligned}
T_{\text {in }} T_{\text {out }}^{\dagger} \propto & \frac{1}{N} \operatorname{Tr}\left(G\left(Y, Y_{1} \mid q_{+}\right) T^{a_{1}} G\left(X_{1}, X_{0} \mid p_{0+}\right) G^{\dagger}\left(\bar{X}, \bar{X}_{0} \mid p_{0+}\right) T^{a}\right) G_{a a_{1}}\left(Z, Z_{1} \mid k_{+}\right) \\
= & \mathcal{B}\left(Z, Z_{1} ; \mathbf{w} \mid k_{+}\right) \mathcal{B}\left(Y, Y_{1} ; \mathbf{r} \mid q_{+}\right) \mathcal{B}\left(X_{1}, X_{0} ; \mathbf{s} \mid p_{0+}\right) \mathcal{B}^{\dagger}\left(\bar{X}, \bar{X}_{0} ; \overline{\mathbf{s}} \mid p_{0+}\right) \\
& \times \frac{1}{2 N}\left\{\operatorname{Tr}\left(W^{\dagger}(\mathbf{w}) W(\mathbf{r})\right) \operatorname{Tr}\left(W(\mathbf{s}) W^{\dagger}(\overline{\mathbf{s}}) W(\mathbf{w})\right)\right. \\
& \left.-\frac{1}{N} \operatorname{Tr}\left(W(\mathbf{s}) W^{\dagger}(\overline{\mathbf{s}}) W(\mathbf{r})\right)\right\}
\end{aligned}
$$


and that the $i n-i n$ one reads

$$
\begin{aligned}
\left|T_{\text {in }}\right|^{2} \propto & \frac{1}{N} \operatorname{Tr}\left(G\left(Y, Y_{1} \mid q_{+}\right) T^{a_{1}} G\left(X_{1}, X_{0} \mid p_{0+}\right) G^{\dagger}\left(\bar{X}_{2}, \bar{X}_{0} \mid p_{0+}\right) T^{\bar{a}_{1}} G^{\dagger}\left(\bar{Y}, \bar{Y}_{2} \mid q_{+}\right)\right) \\
& \times G_{a a_{1}}\left(Z, Z_{1} \mid k_{+}\right) G_{\bar{a}_{1} a}\left(\bar{Z}, \bar{Z}_{2} \mid k_{+}\right) \\
= & \mathcal{B}\left(Z, Z_{1} ; \mathbf{w} \mid k_{+}\right) \mathcal{B}\left(Y, Y_{1} ; \mathbf{r} \mid q_{+}\right) \mathcal{B}\left(X_{1}, X_{0} ; \mathbf{s} \mid p_{0+}\right) \mathcal{B}\left(\bar{Z}, \bar{Z}_{2} ; \overline{\mathbf{w}} \mid k_{+}\right) \\
& \times \mathcal{B}\left(\bar{Y}, \bar{Y}_{2} ; \overline{\mathbf{r}} \mid q_{+}\right) \mathcal{B}\left(\bar{X}_{2}, \bar{X}_{0} ; \overline{\mathbf{s}} \mid p_{0+}\right) \\
& \times \frac{1}{2 N}\left\{\operatorname{Tr}\left(W^{\dagger}(\overline{\mathbf{w}}) W(\mathbf{w}) W(\mathbf{s}) W^{\dagger}(\overline{\mathbf{s}})\right) \operatorname{Tr}\left(W^{\dagger}(\overline{\mathbf{r}}) W(\mathbf{r}) W^{\dagger}(\mathbf{w}) W(\overline{\mathbf{w}})\right)\right. \\
& \left.-\frac{1}{N} \operatorname{Tr}\left(W(\mathbf{s}) W^{\dagger}(\overline{\mathbf{s}}) W^{\dagger}(\overline{\mathbf{r}}) W(\mathbf{r})\right)\right\} .
\end{aligned}
$$

\section{B.2 After region separation}

To simplify further the propagator structure of eq. (2.11), we use the convolution property of the Green's function (eq. (3.1)) to write the propagator of the initial quark in the complex conjugate amplitude as

$$
G_{i j}^{\dagger}\left(\bar{X}, \bar{X}_{0} \mid p_{+}\right)=\int_{\overline{\mathbf{x}}_{1}} G_{i \alpha}^{\dagger}\left(\bar{X}_{1}, \bar{X}_{0} \mid p_{+}\right) G_{\alpha j}^{\dagger}\left(\bar{X}, \bar{X}_{1} \mid p_{+}\right),
$$

where the coordinates are explicitly shown in figure 4. Moreover, due to the locality of the medium averages, the only possible contraction of two fundamental indices, $A$ and $B$, when only two Wilson lines are present in a given local interval, is given by

$$
\left\langle\left(W(\mathbf{x}) W^{\dagger}(\mathbf{y})\right)_{A B}\right\rangle_{\left(x_{f+}, x_{i+}\right)}=\frac{\delta_{A B}}{N}\left\langle\operatorname{Tr}\left(W(\mathbf{x}) W^{\dagger}(\mathbf{y})\right)\right\rangle_{\left(x_{f+}, x_{i+}\right)} .
$$

Thus, from eq. (B.5), one can write

$$
\begin{aligned}
\left\langle T_{\text {in }} T_{\text {out }}^{\dagger}\right\rangle \propto & \mathcal{B}\left(Z, Z_{1} ; \mathbf{w}_{2} \mid k_{+}\right) \mathcal{B}\left(Y, Y_{1} ; \mathbf{r}_{2} \mid q_{+}\right) \mathcal{B}^{\dagger}\left(\bar{X}, \bar{X}_{1} ; \overline{\mathbf{s}}_{2} \mid p_{0+}\right) \mathcal{B}\left(X_{1}, X_{0} ; \mathbf{s}_{1} \mid p_{0+}\right) \\
& \times \mathcal{B}^{\dagger}\left(\bar{X}_{1}, \bar{X}_{0} ; \overline{\mathbf{s}}_{1} \mid p_{0+}\right) \\
& \times \frac{1}{2 N}\left\{\left\langle\operatorname{Tr}\left(W^{\dagger}\left(\mathbf{w}_{2}\right) W\left(\mathbf{r}_{2}\right)\right)\left[W\left(\mathbf{w}_{2}\right) W^{\dagger}\left(\overline{\mathbf{s}}_{2}\right)\right]_{i j}\right\rangle\right. \\
& \left.\times\left\langle\left[W\left(\mathbf{s}_{1}\right) W^{\dagger}\left(\overline{\mathbf{s}}_{1}\right)\right]_{j i}\right\rangle-\frac{1}{N}\left\langle W\left(\mathbf{r}_{2}\right) W^{\dagger}\left(\overline{\mathbf{s}}_{2}\right)\right\rangle_{i j}\left\langle W\left(\mathbf{s}_{1}\right) W^{\dagger}\left(\overline{\mathbf{s}}_{1}\right)\right\rangle_{j i}\right\} \\
= & \mathcal{B}\left(Z, Z_{1} ; \mathbf{w}_{2} \mid k_{+}\right) \mathcal{B}\left(Y, Y_{1} ; \mathbf{r}_{2} \mid q_{+}\right) \mathcal{B}^{\dagger}\left(\bar{X}, \bar{X}_{1} ; \overline{\mathbf{s}}_{2} \mid p_{0+}\right) \mathcal{B}\left(X_{1}, X_{0} ; \mathbf{s}_{1} \mid p_{0+}\right) \\
& \times \mathcal{B}^{\dagger}\left(\bar{X}_{1}, \bar{X}_{0} ; \overline{\mathbf{s}}_{1} \mid p_{0+}\right) \\
& \times \frac{1}{2 N^{2}}\left\{\left\langle\operatorname{Tr}\left(W^{\dagger}\left(\mathbf{w}_{2}\right) W\left(\mathbf{r}_{2}\right)\right) \operatorname{Tr}\left(W\left(\mathbf{w}_{2}\right) W^{\dagger}\left(\overline{\mathbf{s}}_{2}\right)\right)\right\rangle\right. \\
& \times\left\langle\operatorname{Tr}\left(W\left(\mathbf{s}_{1}\right) W^{\dagger}\left(\overline{\mathbf{s}}_{1}\right)\right)\right\rangle \\
& \left.-\frac{1}{N}\left\langle\operatorname{Tr}\left(W\left(\mathbf{r}_{2}\right) W^{\dagger}\left(\overline{\mathbf{s}}_{2}\right)\right)\right\rangle\left\langle\operatorname{Tr}\left(W\left(\mathbf{s}_{1}\right) W^{\dagger}\left(\overline{\mathbf{s}}_{1}\right)\right)\right\rangle\right\}
\end{aligned}
$$


As for the structure of eq. (2.12), assuming that $x_{1+}<x_{2+}$, we need to split the final quark and gluon Green's functions in the amplitude as follows (coordinates are shown in figure 5):

$$
\begin{aligned}
G_{k l}\left(Y, Y_{1} \mid q_{+}\right) & =\int_{\mathbf{y}_{1}} G_{k \beta}\left(Y, Y_{2} \mid q_{+}\right) G_{\beta l}\left(Y_{2}, Y_{1} \mid q_{+}\right), \\
G_{m n}\left(Z, Z_{1} \mid q_{+}\right) & =\int_{\mathbf{z}_{1}} G_{m \delta}\left(Z, Z_{2} \mid k_{+}\right) G_{\delta n}\left(Z_{2}, Z_{1} \mid k_{+}\right) .
\end{aligned}
$$

Using eqs. (B.7) and (B.10), eq. (B.6) can be written

$$
\begin{aligned}
\left\langle\left|T_{\text {in }}\right|^{2}\right\rangle \propto & \mathcal{B}\left(Z, Z_{2} ; \mathbf{w}_{3} \mid k_{+}\right) \mathcal{B}\left(Y, Y_{2} ; \mathbf{r}_{3} \mid q_{+}\right) \mathcal{B}^{\dagger}\left(\bar{Z}, \bar{Z}_{2} ; \overline{\mathbf{w}}_{3} \mid k_{+}\right) \mathcal{B}^{\dagger}\left(\bar{Y}, \bar{Y}_{2} ; \overline{\mathbf{r}}_{3} \mid q_{+}\right) \\
& \times \mathcal{B}\left(Z_{2}, Z_{1} ; \mathbf{w}_{2} \mid k_{+}\right) \mathcal{B}\left(Y_{2}, Y_{1} ; \mathbf{r}_{2} \mid q_{+}\right) \mathcal{B}^{\dagger}\left(\bar{X}_{2}, \bar{X}_{1} ; \overline{\mathbf{s}}_{2} \mid p_{0+}\right) \\
& \times \mathcal{B}\left(X_{1}, X_{0} ; \mathbf{s}_{1} \mid p_{0+}\right) \mathcal{B}^{\dagger}\left(\bar{X}_{1}, \bar{X}_{0} ; \overline{\mathbf{s}}_{1} \mid p_{0+}\right) \\
& \times \frac{1}{2 N}\left\{\left\langle\left[W^{\dagger}\left(\overline{\mathbf{w}}_{3}\right) W\left(\mathbf{w}_{3}\right)\right]_{j i}\left[W^{\dagger}\left(\mathbf{w}_{3}\right) W\left(\overline{\mathbf{w}}_{3}\right) W^{\dagger}\left(\overline{\mathbf{r}}_{3}\right) W\left(\mathbf{r}_{3}\right)\right]_{l k}\right\rangle\right. \\
& \times\left\langle\left[W\left(\mathbf{w}_{2}\right) W^{\dagger}\left(\overline{\mathbf{s}}_{2}\right)\right]_{i j}\left[W\left(\mathbf{r}_{2}\right) W^{\dagger}\left(\mathbf{w}_{2}\right)\right]_{k l}\right\rangle\left\langle\operatorname{Tr}\left(W\left(\mathbf{s}_{1}\right) W^{\dagger}\left(\overline{\mathbf{s}}_{1}\right)\right)\right\rangle \\
& -\frac{1}{N^{3}}\left\langle\operatorname{Tr}\left(W\left(\mathbf{r}_{3}\right) W^{\dagger}\left(\overline{\mathbf{r}}_{3}\right)\right)\right\rangle\left\langle\operatorname{Tr}\left(W\left(\mathbf{r}_{2}\right) W^{\dagger}\left(\overline{\mathbf{s}}_{2}\right)\right)\right\rangle \\
& \left.\times\left\langle\operatorname{Tr}\left(W\left(\mathbf{s}_{1}\right) W^{\dagger}\left(\overline{\mathbf{s}}_{1}\right)\right)\right\rangle\right\} .
\end{aligned}
$$

\section{Medium averages: $n$-point correlators of Wilson lines}

\section{C.1 Two-point correlation function}

The simplest average to be computed is the one involving two fundamental Wilson lines. That is,

$$
\frac{1}{N} \operatorname{Tr}\left\langle W^{F}(\mathbf{x}) W^{\dagger F}(\mathbf{y})\right\rangle=\frac{1}{N}\left\langle W_{i j}(\mathbf{x}) W_{j i}^{\dagger}(\mathbf{y})\right\rangle .
$$

Expanding the Wilson line up to second order in the medium field (see [56]), we find

$$
W_{i j}(\mathbf{x}) \simeq \delta_{i j}+i g \int d x_{+} A_{-}^{a}\left(x_{+}, \mathbf{x}\right) T_{i j}^{a}+\frac{(i g)^{2}}{2}\left(\int d x_{+} A_{-}^{a}\left(x_{+}, \mathbf{x}\right) T_{i j}^{a}\right)^{2}+\ldots
$$

Using this relation, we get

$$
\begin{aligned}
\frac{1}{N} \operatorname{Tr}\left\langle W(\mathbf{x}) W^{\dagger}(\mathbf{y})\right\rangle \simeq 1+(i g)^{2} C_{F} & \left\{\frac{1}{2} \int d x_{+}\left\langle A_{-}\left(x_{+}, \mathbf{x}\right) A_{-}\left(x_{+}, \mathbf{x}\right)\right\rangle\right. \\
& +\frac{1}{2} \int d x_{+}\left\langle A_{-}^{*}\left(y_{+}, \mathbf{y}\right) A_{-}^{*}\left(y_{+}, \mathbf{y}\right)\right\rangle \\
& \left.-\int d x_{+}\left\langle A_{-}\left(x_{+}, \mathbf{x}\right) A_{-}^{*}\left(y_{+}, \mathbf{y}\right)\right\rangle+\ldots\right\}
\end{aligned}
$$

A diagrammatic interpretation of these terms can be found in [38]. 
Fourier transforming the fields and using the fact that all scattering centres are Lorentz contracted in a plane located at $x_{+}$, we get

$$
A_{-}\left(x_{+}, \mathbf{x}\right)=\int \frac{d \mathbf{q}}{(2 \pi)^{2}} \mathrm{e}^{i\left(\mathbf{x}-\mathbf{x}_{i}\right) \cdot \mathbf{q}} a_{-}(\mathbf{q}) \delta\left(x_{+}-x_{i+}\right),
$$

with $\left|a_{-}(\mathbf{q})\right|^{2}$ being a general screened potential (usually taken as a Yukawa potential). In order to perform the average over all possible colour configuration we have to integrate over the transverse and longitudinal coordinates of the scattering centres, $\left(x_{i+}, \mathbf{x}_{i}\right)$. Doing so, we find

$$
\left\langle A_{-}^{a}\left(x_{+}, \mathbf{x}\right) A_{-}^{* b}\left(y_{+}, \mathbf{y}\right)\right\rangle=n\left(x_{+}\right) \delta\left(x_{+}-y_{+}\right) \int \frac{d \mathbf{q}}{(2 \pi)^{2}}\left|a_{-}(\mathbf{q})\right|^{2} \mathrm{e}^{i \mathbf{q} \cdot(\mathbf{x}-\mathbf{y})} \delta^{a b},
$$

where we have introduced the longitudinal density of scattering centres

$$
n\left(x_{+}\right)=\int d x_{i+} \delta\left(x_{+}-x_{i+}\right)
$$

Thus,

$$
\begin{aligned}
\frac{1}{N} \operatorname{Tr}\left\langle W(\mathbf{x}) W^{\dagger}(\mathbf{y})\right\rangle= & 1+(i g)^{2} C_{F} \int d x_{+} \frac{d \mathbf{q}}{(2 \pi)^{2}}\left|a_{-}(\mathbf{q})\right|^{2}\left(1-\mathrm{e}^{i \mathbf{q} \cdot(\mathbf{x}-\mathbf{y})}\right) n\left(x_{+}\right) \\
& +\cdots
\end{aligned}
$$

The dipole cross section is identified as

$$
\sigma(\mathbf{x}-\mathbf{y})=2 g^{2} \int \frac{d \mathbf{q}}{(2 \pi)^{2}}\left|a_{-}(\mathbf{q})\right|^{2}\left(1-\mathrm{e}^{i \mathbf{q} \cdot(\mathbf{x}-\mathbf{y})}\right) .
$$

The result can be re-exponented - with account of the ordering in the $x_{+}$coordinate - due to the fact that there is only one possibility for colour state in this average. We finally find

$$
\frac{1}{N} \operatorname{Tr}\left\langle W(\mathbf{x}) W^{\dagger}(\mathbf{y})\right\rangle=\exp \left\{-\frac{C_{F}}{2} \int d x_{+} \sigma(\mathbf{x}-\mathbf{y}) n\left(x_{+}\right)\right\}
$$

and analogously for the adjoint colour representation, ${ }^{12}$

$$
\frac{1}{N^{2}-1} \operatorname{Tr}\left\langle W^{A}(\mathbf{x}) W^{\dagger A}(\mathbf{y})\right\rangle=\exp \left\{-\frac{C_{A}}{2} \int d x_{+} \sigma(\mathbf{x}-\mathbf{y}) n\left(x_{+}\right)\right\} .
$$

\section{C.2 Four-point correlation function}

The structure that we want to calculate is present in eqs. (3.3) and (3.4). ${ }^{13}$ We write

$$
\begin{aligned}
& \left\langle W_{i j}\left(\mathbf{x}_{1}\right) W_{k l}^{\dagger}\left(\mathbf{x}_{2}\right) W_{m n}\left(\mathbf{x}_{3}\right) W_{o p}^{\dagger}\left(\mathbf{x}_{4}\right)\right\rangle_{\left(L_{+}, x_{+}\right)} \\
& =\left\langle V_{i \alpha}\left(\mathbf{x}_{1}\right) V_{\beta l}^{\dagger}\left(\mathbf{x}_{2}\right) V_{m \mu}\left(\mathbf{x}_{3}\right) V_{\nu p}^{\dagger}\left(\mathbf{x}_{4}\right)\right\rangle \mathcal{T}_{j k n o}^{\alpha \beta \mu \nu} .
\end{aligned}
$$

\footnotetext{
${ }^{12}$ Usually, the colour factor $C_{A}$ is included in the definition of the dipole cross section, $\sigma$.

${ }^{13}$ This and related calculations can be found in [37, 57-59].
} 
To perform the medium average we expand the Wilson lines up to second order in the medium fields (see e.g. [56]), like done in eq. (C.2), but only for the first + light-cone position $\tau$. Doing so, we can write

$$
W_{i j}\left(L_{+}, x_{+} ; \mathbf{x}\right)=V_{i \alpha}\left(L_{+}, \tau ; \mathbf{x}\right)\left[\delta_{\alpha j}\left(1-\frac{C_{F}}{2} B(\tau, \mathbf{0})\right)+i T_{\alpha j}^{a} A^{a}(\tau, \mathbf{x})\right]
$$

where $V\left(L_{+}, \tau ; \mathbf{x}\right)$ denotes the Wilson line from the position $\tau$ to the final extension of the medium and

$$
\delta^{a b} B\left(x_{+} ; \mathbf{x}-\mathbf{y}\right)=\left\langle A^{a}\left(x_{+}, \mathbf{x}\right) A^{b}\left(x_{+}, \mathbf{y}\right)\right\rangle
$$

the correlator between two medium fields. We use the Gaussian approximation in which all information is contained in the two-point function. In the following, we will not write explicitly the + coordinates.

Then the operator $\mathcal{T}_{j k n o}^{\alpha \beta \mu \nu}$ in (C.11) reads

$$
\begin{aligned}
\mathcal{T}_{j k n o}^{\alpha \beta \mu \nu}= & \delta_{\alpha j} \delta_{\beta k} \delta_{\mu n} \delta_{\nu o}\left(1-2 C_{F} B(\mathbf{0})-\frac{1}{2 N}\left[B\left(\mathbf{x}_{1}-\mathbf{x}_{2}\right)-B\left(\mathbf{x}_{1}-\mathbf{x}_{3}\right)\right.\right. \\
& \left.\left.+B\left(\mathbf{x}_{1}-\mathbf{x}_{4}\right)+B\left(\mathbf{x}_{2}-\mathbf{x}_{3}\right)-B\left(\mathbf{x}_{2}-\mathbf{x}_{4}\right)+B\left(\mathbf{x}_{3}-\mathbf{x}_{4}\right)\right]\right) \\
& +\delta_{\alpha j} \delta_{\beta k} \delta_{\mu \nu} \delta_{o n} \frac{1}{2} B\left(\mathbf{x}_{3}-\mathbf{x}_{4}\right)-\delta_{\alpha j} \delta_{\beta o} \delta_{\mu n} \delta_{\nu k} \frac{1}{2} B\left(\mathbf{x}_{2}-\mathbf{x}_{4}\right) \\
& +\delta_{\alpha j} \delta_{\beta \mu} \delta_{k n} \delta_{\nu o} \frac{1}{2} B\left(\mathbf{x}_{2}-\mathbf{x}_{3}\right)+\delta_{\alpha \nu} \delta_{j o} \delta_{\beta k} \delta_{\mu n} \frac{1}{2} B\left(\mathbf{x}_{1}-\mathbf{x}_{4}\right) \\
& -\delta_{\alpha n} \delta_{\beta k} \delta_{\mu j} \delta_{\nu o} \frac{1}{2} B\left(\mathbf{x}_{1}-\mathbf{x}_{3}\right)+\delta_{\alpha \beta} \delta_{j k} \delta_{\mu n} \delta_{\nu o} \frac{1}{2} B\left(\mathbf{x}_{1}-\mathbf{x}_{2}\right) .
\end{aligned}
$$

In the following, we will not work with $B$ 's but with $v$ 's that are related to the dipole cross section:

$$
v(\mathbf{x}-\mathbf{y})=B(\mathbf{0})-B(\mathbf{x}-\mathbf{y}) \equiv \frac{1}{2} \int d x_{+} \sigma(\mathbf{x}-\mathbf{y}) n\left(x_{+}\right) .
$$

We define the following vectors:

$$
\begin{aligned}
& u_{1}=\delta_{j k} \delta_{n o} \rightarrow \delta_{i l} \delta_{m p}, \\
& u_{2}=\delta_{j o} \delta_{n k} \rightarrow \delta_{i p} \delta_{l m},
\end{aligned}
$$

that are not orthogonal:

$$
\begin{aligned}
& u_{1} \cdot u_{1}=u_{2} \cdot u_{2}=N^{2}, \\
& u_{1} \cdot u_{2}=N,
\end{aligned}
$$

so the scalar product is defined through the matrix

$$
\mathcal{G}=\left(\begin{array}{cc}
N^{2} & N \\
N & N^{2}
\end{array}\right) .
$$


One can prove that

$$
\begin{aligned}
& \mathcal{T} u_{1}=\left\{1-\frac{N}{2} O_{1}+\frac{1}{2 N}\left(O_{1}+O_{2}-O_{3}\right)\right\} u_{1}+\frac{1}{2}\left(O_{3}-O_{2}\right) u_{2}, \\
& \mathcal{T} u_{2}=\frac{1}{2}\left(O_{3}-O_{1}\right) u_{1}+\left\{1-\frac{N}{2} O_{2}+\frac{1}{2 N}\left(O_{1}+O_{2}-O_{3}\right)\right\} u_{2},
\end{aligned}
$$

where

$$
\begin{aligned}
& O_{1}=v\left(\mathbf{x}_{1}-\mathbf{x}_{2}\right)+v\left(\mathbf{x}_{3}-\mathbf{x}_{4}\right), \\
& O_{2}=v\left(\mathbf{x}_{1}-\mathbf{x}_{4}\right)+v\left(\mathbf{x}_{3}-\mathbf{x}_{2}\right), \\
& O_{3}=v\left(\mathbf{x}_{1}-\mathbf{x}_{3}\right)+v\left(\mathbf{x}_{2}-\mathbf{x}_{4}\right) .
\end{aligned}
$$

Therefore we can write the operator in the following matrix form:

$$
\mathcal{T}=\left(\begin{array}{cc}
1-\frac{N}{2} O_{1}+\frac{1}{2 N}\left(O_{1}+O_{2}-O_{3}\right) & \frac{1}{2}\left(O_{3}-O_{1}\right) \\
\frac{1}{2}\left(O_{3}-O_{2}\right) & 1-\frac{N}{2} O_{2}+\frac{1}{2 N}\left(O_{1}+O_{2}-O_{3}\right)
\end{array}\right) .
$$

Now we need to act repeatedly with the operator $\mathcal{M}=\mathcal{T}-\mathcal{I}$ on $u_{1}$ (as we have expanded close to this vector that is the one at initial times, (C.12) and (C.14)), and, in order to close the traces, we must project onto $u_{2}$ by doing $u_{2} \mathcal{G M} \mathcal{M}^{n} T u_{1}$ (the + coordinate increases from right to left i.e. $u_{1} \rightarrow u_{2}$ ). We write

$$
\mathcal{M}=\left(\begin{array}{cc}
N m_{11}+\frac{m_{11}^{\prime}}{N} & m_{12} \\
m_{21} & N m_{22}+\frac{m_{22}^{\prime}}{N}
\end{array}\right),
$$

where the matrix elements can be read from (C.21).

At leading $N$, we get

$$
\begin{aligned}
u_{2} \mathcal{G M}^{n} T u_{1} \simeq & N\left[\left.N^{n} m_{11}^{n}\right|_{\left(L_{+}, x_{+}\right)}\right. \\
& \left.+\left.\left.\int_{x_{+}}^{L_{+}} d \tau \sum_{i=0}^{n-1} N^{i} m_{22}^{i}\right|_{\left(L_{+}, \tau\right)} N m_{21}(\tau) N^{n-1-i} m_{11}^{n-1-i}\right|_{\left(\tau, x_{+}\right)}\right],
\end{aligned}
$$

with

$$
\left.m_{22}^{i}\right|_{\left(L_{+}, \tau\right)}=\int_{\tau}^{L_{+}} d \tau_{1} \int_{\tau_{1}}^{L_{+}} d \tau_{2} \ldots \int_{\tau_{i-1}}^{L_{+}} d \tau_{i} m_{22}\left(\tau_{i}\right) \ldots m_{22}\left(\tau_{2}\right) m_{22}\left(\tau_{1}\right)
$$

and analogously for $\left.m_{11}^{n-1-i}\right|_{\left(\tau, x_{+}\right)}$, and $m_{i j}(\tau)$ indicating the integrand in (C.15) evaluated at a given $x_{+}=\tau$. Using the notation

$$
\left.\left.e^{N m_{i i}}\right|_{\left(y_{+}, x_{+}\right)} \equiv \sum_{i=0}^{\infty} m_{i i}^{i}\right|_{\left(y_{+}, x_{+}\right)}
$$

as analogous to a path-ordered exponential between $x_{+}$and $y_{+}$, and taking into account that

$$
\sum_{n=0}^{\infty} \sum_{i=0}^{n-1} \cdots=\sum_{i=0}^{\infty} \sum_{n=i+1}^{\infty} \cdots=\sum_{i=0}^{\infty} \sum_{j(=n-i-1)=0}^{\infty} \cdots
$$


we can make the sum over $n$ to get

$$
\begin{aligned}
\sum_{n=0}^{\infty} u_{2} \mathcal{G M}^{n} T u_{1}= & N\left[\left.e^{N m_{11}}\right|_{\left(L_{+}, x_{+}\right)}\right. \\
& \left.+\left.\left.\int_{x_{+}}^{L_{+}} d \tau e^{N m_{22}}\right|_{\left(L_{+}, \tau\right)} N m_{21}(\tau) e^{N m_{11}}\right|_{\left(\tau, x_{+}\right)}\right]+\mathcal{O}\left(\frac{1}{N}\right) .
\end{aligned}
$$

Using

$$
\left.e^{N m_{i i}}\right|_{\left(c_{+}, a_{+}\right)}=\left.\left.e^{N m_{i i}}\right|_{\left(c_{+}, b_{+}\right)} e^{N m_{i i}}\right|_{\left(b_{+}, a_{+}\right)}, \quad a_{+} \leq b_{+} \leq c_{+}
$$

and

$$
\begin{aligned}
\left.e^{N\left(m_{11}-m_{22}\right)}\right|_{\left(L_{+}, x_{+}\right)} & =1+\left.\int_{x_{+}}^{L_{+}} d \tau \frac{d}{d \tau} e^{N\left(m_{11}-m_{22}\right)}\right|_{\left(\tau, x_{+}\right)} \\
& =1+\left.\int_{x_{+}}^{L_{+}} d \tau N\left(m_{11}-m_{22}\right)(\tau) e^{N\left(m_{11}-m_{22}\right)}\right|_{\left(\tau, x_{+}\right)}
\end{aligned}
$$

eq. (C.27) can be written as

$$
\begin{aligned}
\sum_{n=0}^{\infty} u_{2} \mathcal{G} \mathcal{M}^{n} T u_{1}= & \left.N e^{N m_{22}}\right|_{\left(L_{+}, x_{+}\right)}[1 \\
& \left.-\left.\int_{x_{+}}^{L_{+}} d \tau N\left(m_{22}-m_{11}-m_{21}\right)(\tau) e^{N\left(m_{11}-m_{22}\right)}\right|_{\left(\tau, x_{+}\right)}\right]+\mathcal{O}\left(\frac{1}{N}\right)
\end{aligned}
$$

that is the expression that one would get by making the infinitesimal expansion (C.12) at late times (i.e. close to $u_{2}$ ) as we will do for the six-point function in the next sub appendix (note that $m_{12}=m_{21}+m_{11}-m_{22}$ ).

Let us note that this result is perfectly compatible with those obtained in the strict eikonal limit (with fixed transverse coordinates all along the trajectories of the colour charges) either using the same method [59] or diagonalising the matrix (C.22) as e.g. in [56]. Indeed, considering that the $m_{i j}(\tau)$ do not depend on $\tau$, one gets the result at large $N$ that reads

$$
\begin{aligned}
\sum_{n=0}^{\infty} u_{2} \mathcal{G M}^{n} T u_{1} \simeq & N\left[\left.e^{N m_{22}}\right|_{\left(L_{+}, x_{+}\right)}\right. \\
& \left.+\frac{m_{12}}{m_{22}-m_{11}}\left(\left.e^{N m_{22}}\right|_{\left(L_{+}, x_{+}\right)}-\left.e^{N m_{11}}\right|_{\left(L_{+}, x_{+}\right)}\right)\right] .
\end{aligned}
$$

Finally, these results can be applied to the colour structure in region II. In this case, the structure that we want to simplify is the same as in eqs. (3.3) and (3.4):

$$
\begin{aligned}
& \left\langle\left[W\left(\mathbf{r}_{\mathbf{2}}\right) W^{\dagger}\left(\mathbf{w}_{\mathbf{2}}\right)\right]_{i j}\left[W\left(\mathbf{w}_{\mathbf{2}}\right) W^{\dagger}\left(\overline{\mathbf{s}}_{\mathbf{2}}\right)\right]_{k l}\right\rangle_{\left(x_{2+}, x_{1+}\right)} \\
& =\left\langle W_{i m}\left(\mathbf{r}_{\mathbf{2}}\right) W_{n j}^{\dagger}\left(\mathbf{w}_{\mathbf{2}}\right) W_{k o}\left(\mathbf{w}_{\mathbf{2}}\right) W_{p l}^{\dagger}\left(\overline{\mathbf{s}}_{\mathbf{2}}\right)\right\rangle_{\left(x_{2+}, x_{1+}\right)} \delta_{m n} \delta_{o p} .
\end{aligned}
$$


Here, we must consider the repeated action of $\mathcal{M}$ on $u_{1}$, with the change of notation and the simplifications produced by the fact that two coordinates are equal. The result reads

$$
\begin{aligned}
& \left\langle\left[W\left(\mathbf{r}_{\mathbf{2}}\right) W^{\dagger}\left(\mathbf{w}_{\mathbf{2}}\right)\right]_{i j}\left[W\left(\mathbf{w}_{\mathbf{2}}\right) W^{\dagger}\left(\overline{\mathbf{s}}_{\mathbf{2}}\right)\right]_{k l}\right\rangle_{\left(x_{2+}, x_{1+}\right)} \\
& =\left.e^{N m_{11}+m_{11}^{\prime} / N}\right|_{\left(x_{2+}, x_{1+}\right)} \delta_{i j} \delta_{k l}+\mathcal{O}\left(\frac{1}{N}\right) \\
& =\left.e^{-C_{F}\left[v\left(\mathbf{r}_{2}-\mathbf{w}_{2}\right)+v\left(\mathbf{w}_{2}-\overline{\mathbf{s}}_{2}\right)\right]+\mathcal{O}(1 / N)}\right|_{\left(x_{2+}, x_{1+}\right)}+\mathcal{O}\left(\frac{1}{N}\right) .
\end{aligned}
$$

At large $N$, the result reads:

$$
\begin{aligned}
& \left\langle\left[W\left(\mathbf{r}_{\mathbf{2}}\right) W^{\dagger}\left(\mathbf{w}_{\mathbf{2}}\right)\right]_{i j}\left[W\left(\mathbf{w}_{\mathbf{2}}\right) W^{\dagger}\left(\overline{\mathbf{s}}_{\mathbf{2}}\right)\right]_{k l}\right\rangle_{\left(x_{2+}, x_{1+}\right)} \\
& \underset{N \rightarrow \infty}{\simeq} \frac{1}{N}\left\langle\operatorname{Tr}\left(W\left(\mathbf{r}_{\mathbf{2}}\right) W^{\dagger}\left(\mathbf{w}_{\mathbf{2}}\right)\right)\right\rangle_{\left(x_{2+}, x_{1+}\right)} \frac{1}{N}\left\langle\operatorname{Tr}\left(W\left(\mathbf{w}_{\mathbf{2}}\right) W^{\dagger}\left(\overline{\mathbf{s}}_{\mathbf{2}}\right)\right)\right\rangle_{\left(x_{2+}, x_{1+}\right)} .
\end{aligned}
$$

\section{C.3 Six-point correlation function}

For the purposes of the main body of this work, it suffices to evaluate the six-point correlation function appearing in (3.4) in the large $N$ limit. Following the procedure already adopted for the four-point case, we write

$$
\left\langle W_{i j}^{\dagger}\left(\overline{\mathbf{w}}_{3}\right) W_{k l}\left(\mathbf{w}_{3}\right) W_{m n}^{\dagger}\left(\mathbf{w}_{3}\right) W_{o p}\left(\overline{\mathbf{w}}_{3}\right) W_{q r}^{\dagger}\left(\overline{\mathbf{r}}_{3}\right) W_{s t}\left(\mathbf{r}_{3}\right)\right\rangle_{\left(L_{+}, x_{2+}\right)}
$$

and define the colour contractions

$$
\begin{aligned}
& u_{1}=\delta_{j k} \delta_{n o} \delta_{r s}, \\
& u_{2}=\delta_{j s} \delta_{r k} \delta_{n o}, \\
& u_{3}=\delta_{j o} \delta_{n k} \delta_{r s}, \\
& u_{4}=\delta_{j o} \delta_{r k} \delta_{n s}, \\
& u_{5}=\delta_{j s} \delta_{n k} \delta_{r o}, \\
& u_{6}=\delta_{j k} \delta_{n s} \delta_{r o},
\end{aligned}
$$

with scalar products given through the matrix

$$
\mathcal{G}=\left(\begin{array}{cccccc}
N^{3} & N^{2} & N^{2} & N & N & N^{2} \\
N^{2} & N^{3} & N & N^{2} & N^{2} & N \\
N^{2} & N & N^{3} & N^{2} & N^{2} & N \\
N & N^{2} & N^{2} & N^{3} & N & N^{2} \\
N & N^{2} & N^{2} & N & N^{3} & N^{2} \\
N^{2} & N & N & N^{2} & N^{2} & N^{3}
\end{array}\right) .
$$

Using the same technique discussed in appendix C.2 but now making the infinitesimal expansion (C.12) at late times, the matrix operator that expresses the medium averages in 
this basis in the Gaussian approximation reads

$$
\mathcal{T}=\mathcal{I}_{6 \times 6}
$$

$$
+\left(\begin{array}{cccccc}
N m_{11}+\frac{m_{11}^{\prime}}{N} & m_{12} & 0 & 0 & 0 & m_{16} \\
m_{21} & N m_{22}+\frac{m_{22}^{\prime}}{N} & 0 & 0 & 0 & 0 \\
m_{31} & 0 & N m_{33}+\frac{m_{33}^{\prime}}{N} & m_{34} & m_{35} & 0 \\
0 & m_{42} & 0 & N m_{44}+\frac{m_{44}^{\prime}}{N} & 0 & m_{46} \\
0 & m_{52} & 0 & 0 & N m_{55}+\frac{m_{55}^{\prime}}{N} & m_{56} \\
m_{61} & 0 & 0 & 0 & 0 & N m_{66}+\frac{m_{66}^{\prime}}{N}
\end{array}\right),
$$

with

$$
\begin{aligned}
& m_{11}=-\frac{1}{2}\left[v\left(\mathbf{r}_{3}-\overline{\mathbf{r}}_{3}\right)+2 v\left(\mathbf{w}_{3}-\overline{\mathbf{w}}_{3}\right)\right], \\
& m_{12}=\frac{1}{2}\left[-v\left(\mathbf{r}_{3}-\overline{\mathbf{r}}_{3}\right)+v\left(\mathbf{r}_{3}-\mathbf{w}_{3}\right)+v\left(\overline{\mathbf{r}}_{3}-\overline{\mathbf{w}}_{3}\right)-v\left(\mathbf{w}_{3}-\overline{\mathbf{w}}_{3}\right)\right], \\
& m_{16}=\frac{1}{2}\left[-v\left(\mathbf{r}_{3}-\overline{\mathbf{r}}_{3}\right)+v\left(\mathbf{r}_{3}-\overline{\mathbf{w}}_{3}\right)+v\left(\overline{\mathbf{r}}_{3}-\mathbf{w}_{3}\right)-v\left(\mathbf{w}_{3}-\overline{\mathbf{w}}_{3}\right)\right], \\
& m_{21}=\frac{1}{2}\left[v\left(\mathbf{r}_{3}-\mathbf{w}_{3}\right)-v\left(\mathbf{r}_{3}-\overline{\mathbf{w}}_{3}\right)-v\left(\overline{\mathbf{r}}_{3}-\mathbf{w}_{3}\right)+v\left(\overline{\mathbf{r}}_{3}-\overline{\mathbf{w}}_{3}\right)\right]=-m_{61}, \\
& m_{22}=-\frac{1}{2}\left[v\left(\mathbf{r}_{3}-\overline{\mathbf{w}}_{3}\right)+v\left(\overline{\mathbf{r}}_{3}-\mathbf{w}_{3}\right)+v\left(\mathbf{w}_{3}-\overline{\mathbf{w}}_{3}\right)\right], \\
& m_{31}=\frac{1}{2} v\left(\mathbf{w}_{3}-\overline{\mathbf{w}}_{3}\right), m_{33}=-\frac{1}{2} v\left(\mathbf{r}_{3}-\overline{\mathbf{r}}_{3}\right), \\
& m_{34}=\frac{1}{2}\left[-v\left(\mathbf{r}_{3}-\overline{\mathbf{r}}_{3}\right)+v\left(\mathbf{r}_{3}-\mathbf{w}_{3}\right)+v\left(\overline{\mathbf{r}}_{3}-\mathbf{w}_{3}\right)\right], \\
& m_{35}=\frac{1}{2}\left[-v\left(\mathbf{r}_{3}-\overline{\mathbf{r}}_{3}\right)+v\left(\mathbf{r}_{3}-\overline{\mathbf{w}}_{3}\right)+v\left(\overline{\mathbf{r}}_{3}-\overline{\mathbf{w}}_{3}\right)\right], \\
& m_{42}=\frac{1}{2}\left[-v\left(\mathbf{r}_{3}-\mathbf{w}_{3}\right)+v\left(\mathbf{r}_{3}-\overline{\mathbf{w}}_{3}\right)+v\left(\mathbf{w}_{3}-\overline{\mathbf{w}}_{3}\right)\right], \\
& m_{44}=-\frac{1}{2}\left[v\left(\mathbf{r}_{3}-\mathbf{w}_{3}\right)+v\left(\overline{\mathbf{r}}_{3}-\mathbf{w}_{3}\right)\right], \\
& m_{46}=\frac{1}{2}\left[-v\left(\overline{\mathbf{r}}_{3}-\mathbf{w}_{3}\right)+v\left(\overline{\mathbf{r}}_{3}-\overline{\mathbf{w}}_{3}\right)+v\left(\mathbf{w}_{3}-\overline{\mathbf{w}}_{3}\right)\right], \\
& m_{52}=\frac{1}{2}\left[v\left(\overline{\mathbf{r}}_{3}-\mathbf{w}_{3}\right)-v\left(\overline{\mathbf{r}}_{3}-\overline{\mathbf{w}}_{3}\right)+v\left(\mathbf{w}_{3}-\overline{\mathbf{w}}_{3}\right)\right], \\
& m_{55}=-\frac{1}{2}\left[v\left(\mathbf{r}_{3}-\overline{\mathbf{w}}_{3}\right)+v\left(\overline{\mathbf{r}}_{3}-\overline{\mathbf{w}}_{3}\right)\right], \\
& m_{56}=\frac{1}{2}\left[v\left(\mathbf{r}_{3}-\mathbf{w}_{3}\right)-v\left(\mathbf{r}_{3}-\overline{\mathbf{w}}_{3}\right)+v\left(\mathbf{w}_{3}-\overline{\mathbf{w}}_{3}\right)\right], \\
& =\frac{1}{2}\left[v\left(\mathbf{r}_{3}-\mathbf{w}_{3}\right)+v\left(\overline{\mathbf{r}}_{3}-\overline{\mathbf{w}}_{3}\right)+v\left(\mathbf{w}_{3}-\overline{\mathbf{w}}_{3}\right)\right], \\
& m_{33}^{\prime}=m_{44}^{\prime}=m_{55}^{\prime}=m_{66}^{\prime}=\frac{1}{2} v\left(\mathbf{r}_{3}-\overline{\mathbf{r}}_{3}\right) .
\end{aligned}
$$

Then, to perform the medium average in region III in (3.4), we compute $n$ insertions of the matrix $\mathcal{M}=\mathcal{T}-\mathcal{I}_{6 \times 6}$ (see e.g. [60]), project onto the corresponding vectors and 
sum over $n$ i.e.

$$
\left\langle\operatorname{Tr}\left(W^{\dagger}\left(\overline{\mathbf{w}}_{3}\right) W\left(\mathbf{w}_{3}\right)\right) \operatorname{Tr}\left(W^{\dagger}\left(\mathbf{w}_{3}\right) W\left(\overline{\mathbf{w}}_{3}\right) W^{\dagger}\left(\overline{\mathbf{r}}_{3}\right) W\left(\mathbf{r}_{3}\right)\right)\right\rangle_{\left(L_{+}, x_{2+}\right)}=\sum_{n=0}^{\infty} u_{6} \mathcal{G} \mathcal{M}^{n} u_{1}
$$

with the + coordinate increasing from left to right i.e. $u_{6} \rightarrow u_{1}$ in this case (see the comment below (C.30)).

Successive insertions of $\mathcal{M}$ can be understood as either propagating a given colour structure $u_{i}$ (in the form of an insertion of diagonal matrix elements $m_{i i}$ ) or as swapping colour structure from $u_{i}$ into $u_{j}$ (with off-diagonal matrix element $m_{j i}$ ). As each diagonal matrix element (we will discuss their $1 / N$ corrections later on) carries a factor of $N$ and the projection of the leftmost colour structure (given by the leftmost of swaps) onto $u_{6}$ contributes with $N^{p}, p=1,2,3$, according to (C.37), a term in (C.40) with $n$ insertions of which $s$ are colour swaps will carry an overall power of $N$ given by $N^{n-s+p}$ which we write as $N^{p-s} N^{n}$.

The leading order result is obtained from terms with no swaps, $s=0$, (the diagonal propagation of the colour structure $u_{1}$ ) for which the final projection is $u_{6} \cdot u_{1}=N^{2}(p=2)$, and terms with one swap, $s=1, m_{61}$ from $u_{1}$ to $u_{6}$ for which the final projection yields $u_{6} \cdot u_{6}=N^{3}(p=3)$ :

$$
\begin{aligned}
& \left\langle\operatorname{Tr}(\cdot \cdot \operatorname{Tr}(\cdot \cdot \cdot)\rangle_{\mathcal{O}\left(N^{2}\right) e^{\mathcal{O}(N)}}\right. \\
& =N^{2} \sum_{n=0}^{\infty} N^{n}\left(\left.m_{11}^{n}\right|_{\left(L_{+}, x_{2+}\right)}+\left.\left.\int_{x_{2+}}^{L_{+}} d \tau \sum_{i=0}^{n-1} m_{11}^{n-1-i}\right|_{\left(L_{+}, \tau\right)} m_{61}(\tau) m_{66}^{i}\right|_{\left(\tau, x_{2+}\right)}\right) \\
& =N^{2}\left(\left.e^{N m_{11}}\right|_{\left(L_{+}, x_{2+}\right)}+\left.\left.\int_{x_{2+}}^{L_{+}} d \tau e^{N m_{11}}\right|_{\left(L_{+}, \tau\right)} N m_{61}(\tau) e^{N m_{66}}\right|_{\left(\tau, x_{2+}\right)}\right),
\end{aligned}
$$

using (C.24), (C.25) and (C.26). This expression corresponds to the factorisation of the six-point function into a dipole and a quadrupole with the latter given in the large- $N$ limit by $($ C. .30$):{ }^{14}$

$$
\begin{gathered}
\left\langle\operatorname{Tr}\left(W^{\dagger}\left(\overline{\mathbf{w}}_{3}\right) W\left(\mathbf{w}_{3}\right)\right) \operatorname{Tr}\left(W^{\dagger}\left(\mathbf{w}_{3}\right) W\left(\overline{\mathbf{w}}_{3}\right) W^{\dagger}\left(\overline{\mathbf{r}}_{3}\right) W\left(\mathbf{r}_{3}\right)\right)\right\rangle_{\left(L_{+}, x_{2+}\right)} \\
\underset{N \rightarrow \infty}{\simeq}\left\langle\operatorname{Tr}\left(W^{\dagger}\left(\overline{\mathbf{w}}_{3}\right) W\left(\mathbf{w}_{3}\right)\right)\right\rangle_{\left(L_{+}, x_{2+}\right)}\left\langle\operatorname{Tr}\left(W^{\dagger}\left(\mathbf{w}_{3}\right) W\left(\overline{\mathbf{w}}_{3}\right) W^{\dagger}\left(\overline{\mathbf{r}}_{3}\right) W\left(\mathbf{r}_{3}\right)\right)\right\rangle_{\left(L_{+}, x_{2+}\right)} .
\end{gathered}
$$

It is straightforward to see that the subleading corrections $m_{i i}^{\prime}$ in the diagonal matrix elements can be recovered through the substitution $m_{i i} \rightarrow m_{i i}+m_{i i}^{\prime} / N^{2}$.

\section{Path integrals in the multiple soft scattering approximation}

The path integrals that appear can be solved analytically for very few examples. Here we use the semi-classical approximation [61,62], which consists in considering the path integral

\footnotetext{
${ }^{14}$ Note the common factor $v\left(\mathbf{w}_{3}-\overline{\mathbf{w}}_{3}\right)$ in $m_{11}$ and $m_{66}$ and, in order to see the exact equivalence with (C.30), make the substitutions $m_{11} \rightarrow m_{22}$ and $m_{66} \rightarrow m_{11}$, and keep in mind that $m_{61}=m_{21}+$ $m_{11}-m_{22}=m_{12}$.
} 
as the classical action. As a consequence, the trajectory of the particle inside the medium is considered to be the classical path in the exponent, while fluctuations are considered in the norm.

The semi-classical method provides an exact solution for some cases e.g. the free particle or the harmonic oscillator that, in order to clarify subsequent computations, we elaborate as a first step.

\section{D.1 Semi-classical method and some general examples}

The trajectory of free particles is described by the following propagator:

$$
G_{0}\left(y_{+}, \mathbf{y} ; x_{+}, \mathbf{x} \mid p_{+}\right)=\int_{\mathbf{r}\left(x_{+}\right)=\mathbf{x}}^{\mathbf{r}\left(y_{+}\right)=\mathbf{y}} \mathcal{D} \mathbf{r}(\xi) \exp \left\{\frac{i p_{+}}{2} \int_{x_{+}}^{y_{+}} d \xi \dot{\mathbf{r}}^{2}\right\},
$$

with $\dot{\mathbf{r}}=d \mathbf{r}(\xi) / d \xi$. In the semi-classical approximation, we identify the above expression with

$$
G_{0}\left(y_{+}, \mathbf{y} ; x_{+}, \mathbf{y} \mid p_{+}\right) \propto \exp \left\{i R_{\mathrm{cl}}\left(\mathbf{r}_{\mathrm{cl}}\right)\right\},
$$

where the classical action $R_{\mathrm{cl}}$ is defined as

$$
R_{\mathrm{cl}}=\int_{x_{+}}^{y_{+}} d \xi \mathcal{L}(\mathbf{r}, \dot{\mathbf{r}}, \xi)
$$

and the Lagrangian is

$$
\mathcal{L}_{\text {free }}(\mathbf{r}, \dot{\mathbf{r}}, \xi)=\frac{p_{+}}{2} \dot{\mathbf{r}}^{2}
$$

Using the Euler-Lagrange equations, we find

$$
\frac{d}{d t} \frac{\partial \mathcal{L}}{\partial \dot{\mathbf{r}}}-\frac{\partial \mathcal{L}}{\partial \mathbf{r}}=0 \Leftrightarrow \ddot{\mathbf{r}}=0
$$

so the classical trajectory $\mathbf{r}(\xi)$ is given by a straight line:

$$
\mathbf{r}(\xi)=\mathbf{l}(\xi)=\frac{1}{y_{+}-x_{+}}\left[\mathbf{y}\left(\xi-x_{+}\right)+\mathbf{x}\left(y_{+}-\xi\right)\right]
$$

The solution for the path integral $(2.3)$ is $[61,62]$

$$
G_{0}=\frac{1}{(2 \pi i)^{D / 2}}\left|\operatorname{det}\left(-\frac{\partial^{2} R_{\mathrm{cl}}}{\partial \mathbf{y}_{i} \partial \mathbf{x}_{j}}\right)\right|^{1 / 2} \exp \left[i R_{\mathrm{cl}}\left(x_{+}, \mathbf{x} ; y_{+}, \mathbf{y}\right)\right],
$$

where $D$ is the number of dimensions, $i, j \in\{1, \ldots, D\}$ and $R_{\mathrm{cl}}$ is evaluated at the classical path $\mathbf{r}=\mathbf{l}(\xi)$ and integrated over $\xi \in\left[x_{+}, y_{+}\right]$. The derivatives are taken on the initial and final transverse coordinates. In our case, $D=2$ and the result of the action is

$$
R_{\mathrm{cl}}=\int_{x_{+}}^{y_{+}} d \xi \mathcal{L}=\frac{p_{+}}{2} \frac{(\mathbf{y}-\mathbf{x})^{2}}{\left(y_{+}-x_{+}\right)}
$$

resulting in a determinant

$$
\operatorname{det}\left(-\frac{\partial^{2} R_{\mathrm{cl}}}{\partial \mathbf{y}_{\mathbf{i}} \partial \mathbf{x}_{j}}\right)=\left(\frac{p_{+}}{y_{+}-x_{+}}\right)^{2}
$$


So, one finally finds

$$
G_{0}\left(y_{+}, \mathbf{y} ; x_{+}, \mathbf{x} \mid p_{+}\right)=\frac{p_{+}}{2 \pi i\left(y_{+}-x_{+}\right)} \exp \left\{\frac{i p_{+}}{2} \frac{(\mathbf{y}-\mathbf{x})^{2}}{\left(y_{+}-x_{+}\right)}\right\} .
$$

Another example is the harmonic oscillator:

$$
\begin{aligned}
\mathcal{K}_{\mathrm{osc}}\left(y_{+}, \mathbf{y} ; x_{+}, \mathbf{x} \mid p_{+}\right) & =\frac{1}{N}\left\langle\operatorname{Tr}\left(G\left(y_{+}, \mathbf{y} ; x_{+}, \mathbf{x} \mid p_{+}\right) W^{\dagger}\left(y_{+}, x_{+}, \mathbf{0}\right)\right)\right\rangle \\
& =\int_{\mathbf{r}\left(x_{+}\right)=\mathbf{x}}^{\mathbf{r}\left(y_{+}\right)=\mathbf{y}} \mathcal{D} \mathbf{r}(\xi) \exp \left\{\frac{i p_{+}}{2} \int_{x_{+}}^{y_{+}} d \xi \dot{\mathbf{r}}^{2}-\frac{\hat{q}_{F}}{4} \int_{x_{+}}^{y_{+}} d \xi \mathbf{r}^{2}\right\},
\end{aligned}
$$

where it was used the multiple soft scattering approximation (eq. (3.9)). The corresponding Lagrangian is:

$$
\mathcal{L}_{\mathrm{HO}}=\frac{p_{+}}{2} \dot{\mathbf{r}}^{2}+i \frac{\hat{q}_{F}}{4} \mathbf{r}^{2},
$$

that results in the following equation of motion:

$$
\ddot{\mathbf{r}}+\Omega^{2} \mathbf{r}=0
$$

with imaginary frequency:

$$
\Omega^{2}=-i \frac{\hat{q}_{F}}{2 p_{+}}
$$

The solution is

$$
\mathbf{r}(\xi)=\frac{1}{\sin \left[\Omega\left(y_{+}-x_{+}\right)\right]}\left\{\mathbf{x} \sin \left[\Omega\left(y_{+}-\xi\right)\right]+\mathbf{y} \sin \left[\Omega\left(\xi-x_{+}\right)\right]\right\} .
$$

Plugin this solution into the classical action, we find

$$
R_{\mathrm{cl}}=A B\left(\mathbf{x}^{2}+\mathbf{y}^{2}\right)-2 A \mathbf{x} \cdot \mathbf{y},
$$

with

$$
\begin{aligned}
A & =\frac{p_{+} \Omega}{2 \sin \left[\Omega\left(y_{+}-x_{+}\right)\right]}, \\
B & =\cos \left[\Omega\left(y_{+}-x_{+}\right)\right] .
\end{aligned}
$$

It is easy to show that in this case:

$$
\operatorname{det}\left(-\frac{\partial^{2} R_{\mathrm{cl}}}{\partial \mathbf{y} \partial \mathbf{x}}\right)=4 A^{2}
$$

Thus, the solution for the harmonic oscillator is the well-known result

$$
\mathcal{K}_{\mathrm{osc}}\left(y_{+}, \mathbf{y} ; x_{+}, \mathbf{x} \mid p_{+}\right)=\frac{A}{\pi i} \exp \left\{i A B\left(\mathbf{x}^{2}+\mathbf{y}^{2}\right)-2 i A \mathbf{x} \cdot \mathbf{y}\right\} .
$$




\section{D.2 Region I: two path integrals}

The region I is shared by the tree contributions to the total spectrum, eq.(2.5), only changing the ending coordinates (see figure 3 and eqs. (3.2), (3.3) and (3.8)):

$$
\begin{gathered}
\Sigma_{1}\left(x_{0+}, x_{1+}, \mathbf{x}_{0}, \overline{\mathbf{x}}_{0}, \mathbf{x}_{1}, \overline{\mathbf{x}}_{1}\right)=\frac{1}{N}\left\langle\operatorname{Tr}\left(G\left(X_{0}, X_{1} \mid p_{0+}\right) G^{\dagger}\left(\bar{X}_{0}, \bar{X}_{1} \mid p_{0+}\right)\right)\right\rangle \\
=\int_{X_{0}}^{X_{1}} \mathcal{D} \mathbf{s}_{1}\left(\xi_{1}\right) \int_{\left.\bar{X}_{0+}, x_{1+}\right)}^{\bar{X}_{1}} \mathcal{D} \overline{\mathbf{s}}_{1}\left(\xi_{1}\right) \exp \left\{\frac{i p_{0+}}{2} \int_{\xi_{1}}\left(\dot{\mathbf{s}}_{1}^{2}-\dot{\mathbf{s}}_{1}^{2}\right)-\frac{\hat{q}_{F}}{4} \int_{\xi_{1}}\left(\mathbf{s}_{1}-\overline{\mathbf{s}}_{1}\right)^{2}\right\},
\end{gathered}
$$

where $\xi_{1} \in\left[x_{0+}, x_{1+}\right]$. In this expression, both $\mathbf{s}_{1}$ and $\overline{\mathbf{s}}_{1}$ enter the kinematical and potential terms. Performing the following change of variables:

$$
\begin{aligned}
& \mathbf{u}_{1}=\mathbf{s}_{1}-\overline{\mathbf{s}}_{1}, \\
& \mathbf{v}_{1}=\mathbf{s}_{1}+\overline{\mathbf{s}}_{1},
\end{aligned}
$$

we can write the potential term with a dependency on one single variable. Moreover, using Fujikawa's method [63], the change of variables in path integrals comes with the inverse Jacobian of the transformation matrix:

$$
\int \mathcal{D} \mathbf{s}_{1} \mathcal{D} \overline{\mathbf{s}}_{1}=\operatorname{det}\left(\frac{\partial\left(\mathbf{u}_{1}, \mathbf{v}_{1}\right)}{\partial\left(\mathbf{s}_{1}, \overline{\mathbf{s}}_{1}\right)}\right) \int \mathcal{D} \mathbf{u}_{1} \mathcal{D} \mathbf{v}_{1}
$$

where

$$
\frac{\partial\left(\mathbf{u}_{1}, \mathbf{v}_{1}\right)}{\partial\left(\mathbf{s}_{1}, \overline{\mathbf{s}}_{1}\right)}=\left(\begin{array}{ll}
\frac{\partial \mathbf{u}_{1}}{\partial \mathbf{s}_{1}} & \frac{\partial \mathbf{u}_{1}}{\partial \mathbf{s}_{1}} \\
\frac{\partial \mathbf{v}_{1}}{\partial \mathbf{s}_{1}} & \frac{\partial \mathbf{v}_{1}}{\partial \mathbf{s}_{1}}
\end{array}\right)=\left(\begin{array}{cccc}
1 & 0 & -1 & 0 \\
0 & 1 & 0 & -1 \\
1 & 0 & 1 & 0 \\
0 & 1 & 0 & 1
\end{array}\right) \Rightarrow \operatorname{det}\left(\frac{\partial\left(\mathbf{u}_{1}, \mathbf{v}_{1}\right)}{\partial\left(\mathbf{s}_{1}, \overline{\mathbf{s}}_{1}\right)}\right)=4
$$

Therefore, omitting the time dependency,

$$
\Sigma_{1}\left(\mathbf{x}_{0}, \overline{\mathbf{x}}_{0}, \mathbf{x}_{1}, \overline{\mathbf{x}}_{1}\right)=4 \int_{\mathbf{u}_{i}}^{\mathbf{u}_{f}} \mathcal{D} \mathbf{u} \int_{\mathbf{v}_{i}}^{\mathbf{v}_{f}} \mathcal{D} \mathbf{v} \exp \left\{\frac{i p_{0+}}{2} \int_{\xi_{1}} \dot{\mathbf{u}}_{1} \cdot \dot{\mathbf{v}}_{1}-\frac{\hat{q}_{F}}{4} \int_{\xi_{1}} \mathbf{u}_{1}^{2}\right\},
$$

where

$$
\begin{aligned}
\mathbf{u}_{1 i} & =\mathbf{x}_{0}-\overline{\mathbf{x}}_{0}, & \mathbf{v}_{1 i} & =\mathbf{x}_{0}+\overline{\mathbf{x}}_{0}, \\
\mathbf{u}_{1 f} & =\mathbf{x}_{1}-\overline{\mathbf{x}}_{1}, & \mathbf{v}_{1 f} & =\mathbf{x}_{1}+\overline{\mathbf{x}}_{1} .
\end{aligned}
$$

This allow us to find the equations of Euler-Lagrange that constrain $\mathbf{u}_{1}$ to be the same as the free particle (eq. (D.6)):

$$
\mathbf{u}_{1}\left(\xi_{1}\right)=\mathbf{l}_{1}\left(\xi_{1}\right)=\frac{1}{\Delta \xi_{1}}\left[\mathbf{u}_{1 f}\left(\xi_{1}-x_{0+}\right)+\mathbf{u}_{1 i}\left(x_{1+}-\xi_{1}\right)\right],
$$

where $\Delta \xi_{1}=x_{1+}-x_{0+}$.

As for the evolution of the norm, since one of the path integrals comes from a complex conjugate propagator:

$$
\int \mathcal{D} \mathbf{u}_{1} \mathcal{D} \mathbf{v}_{1}=\frac{1}{2 \pi i} \frac{1}{(-2 \pi i)}\left|\operatorname{det}\left(-\frac{\partial^{2} R_{\mathrm{cl}}}{\partial_{(\mathbf{f})} \partial_{(\mathbf{i})}}\right)\right|=\left(\frac{p_{0+}}{4 \pi \Delta \xi_{1}}\right)^{2}
$$


where $\partial_{(\mathbf{f})}$ and $\partial_{(\mathbf{i})}$ are the derivatives with respect to all final and initial coordinates (formed by the 2-vectors $\left(\mathbf{u}_{1 f}, \mathbf{v}_{1 f}\right)$ and $\left(\mathbf{u}_{1 i}, \mathbf{v}_{1 i}\right)$ ).

Putting all results together,

$$
\begin{aligned}
& \Sigma_{1}\left(x_{0+}, x_{1+}, \mathbf{x}_{0}, \overline{\mathbf{x}}_{0}, \mathbf{x}_{1}, \overline{\mathbf{x}}_{1}\right) \\
& \quad=\left(\frac{p_{0+}}{2 \pi \Delta \xi_{1}}\right)^{2} \exp \left\{\frac{i p_{0+}}{2 \Delta \xi_{1}} \Delta \mathbf{l}_{1} \cdot \Delta \mathbf{v}_{1}-\frac{\hat{q}_{F} \Delta \xi_{1}}{12}\left(\mathbf{l}_{1 i}^{2}+\mathbf{l}_{1 f}^{2}+\mathbf{l}_{1 i} \cdot \mathbf{l}_{1 f}\right)\right\},
\end{aligned}
$$

where $\Delta \mathbf{l}_{1}=\mathbf{l}_{1 f}-\mathbf{l}_{1 i}$, and $\Delta \mathbf{v}_{1}=\mathbf{v}_{1 f}-\mathbf{v}_{1 i}$.

\section{D.3 Region II: three path integrals}

Region II is shared by the in - out and in - in contributions, only differing in the ending coordinates (see figure 3 and eqs. (3.3) and (3.8)):

$$
\begin{aligned}
& \Sigma_{2}(\left.x_{1+}, x_{2+}, \mathbf{y}_{1}, \mathbf{z}_{1}, \overline{\mathbf{x}}_{1}, \mathbf{y}_{2}, \mathbf{z}_{2}, \overline{\mathbf{x}}_{2}\right)=\frac{1}{N^{2}} \int_{Z_{1}}^{Z_{2}} \mathcal{D} \mathbf{w}_{2} \exp \left\{\frac{i \zeta p_{+}}{2} \int^{\xi_{2}} \dot{\mathbf{w}}_{2}^{2}\right\} \\
& \quad \times \operatorname{Tr}\left\langle G\left(Y_{2}, Y_{1} \mid q_{+}\right) W^{\dagger}\left(\mathbf{w}_{2}\right)\right\rangle_{\left(x_{2+}, x_{1+}\right)} \operatorname{Tr}\left\langle G^{\dagger}\left(\bar{X}_{2}, \bar{X}_{1} \mid p_{0+}\right) W\left(\mathbf{w}_{2}\right)\right\rangle \\
&=\int_{\left.Z_{1}, x_{1+}\right)}^{Z_{2}} \mathcal{D} \mathbf{w}_{2}\left(\xi_{2}\right) \int_{Y_{1}}^{Y_{2}} \mathcal{D} \mathbf{r}_{2}\left(\xi_{2}\right) \int_{\bar{X}_{1}}^{\bar{X}_{2}} \mathcal{D} \overline{\mathbf{s}}_{2}\left(\xi_{2}\right) \exp \left\{\frac{i p_{+}}{2}\right. \\
&\left.\quad \int^{\xi_{2}}\left[\zeta \dot{\mathbf{w}}_{2}^{2}+(1-\zeta) \dot{\mathbf{r}}_{2}^{2}-\dot{\overline{\mathbf{s}}}_{2}^{2}\right]-\frac{\hat{q}_{F}}{4} \int^{\xi_{2}}\left[\left(\mathbf{r}_{2}-\mathbf{w}_{2}\right)^{2}+\left(\mathbf{w}_{2}-\overline{\mathbf{s}}_{2}\right)^{2}\right]\right\} .
\end{aligned}
$$

Performing the following change of variables with unitary Jacobian:

$$
\begin{aligned}
& \mathbf{u}_{2}=\mathbf{r}_{2}-\mathbf{w}_{2}, \\
& \mathbf{v}_{2}=\zeta \mathbf{w}_{2}+(1-\zeta) \mathbf{r}_{2}-\overline{\mathbf{s}}_{2},
\end{aligned}
$$

the kinetic term becomes linear in $\overline{\mathbf{s}}_{2}$. Omitting the time dependence,

$$
\begin{aligned}
\Sigma_{2}\left(\mathbf{y}_{1}, \mathbf{z}_{1}, \overline{\mathbf{x}}_{1}, \mathbf{y}_{2}, \mathbf{z}_{2}, \overline{\mathbf{x}}_{2}\right)=\int_{\mathbf{u}_{2 i}}^{\mathbf{u}_{2 f}} \mathcal{D} \mathbf{u}_{2} \int_{\mathbf{v}_{2 i}}^{\mathbf{v}_{\mathbf{2 f}}} \mathcal{D} \mathbf{v}_{2} \int_{\overline{\mathbf{s}}_{2 i}}^{\overline{\mathbf{s}}_{2 f}} \mathcal{D} \overline{\mathbf{s}}_{2} \exp \left\{\frac{i p_{0+}}{2} \int^{\xi_{2}}\right. \\
\left.\times\left[2 \dot{\overline{\mathbf{s}}}_{2} \cdot \dot{\mathbf{v}}_{2}+\dot{\mathbf{v}}_{2}^{2}+\zeta(1-\zeta) \dot{\mathbf{u}}_{2}^{2}\right]-\frac{\hat{q}_{F}}{4} \int^{\xi_{2}}\left[\mathbf{u}_{2}^{2}+\left(\mathbf{v}_{2}-(1-\zeta) \mathbf{u}_{2}\right)^{2}\right]\right\},
\end{aligned}
$$

with

$$
\begin{aligned}
& \mathbf{u}_{2 i}=\mathbf{y}_{1}-\mathbf{z}_{1}, \quad \mathbf{v}_{2 i}=\zeta \mathbf{z}_{1}+(1-\zeta) \mathbf{y}_{1}-\overline{\mathbf{x}}_{1}, \quad \overline{\mathbf{s}}_{2 i}=\overline{\mathbf{x}}_{1}, \\
& \mathbf{u}_{2 f}=\mathbf{y}_{2}-\mathbf{z}_{2}, \quad \mathbf{v}_{2 f}=\zeta \mathbf{z}_{2}+(1-\zeta) \mathbf{y}_{2}-\overline{\mathbf{x}}_{2}, \quad \overline{\mathbf{s}}_{2 f}=\overline{\mathbf{x}}_{2} .
\end{aligned}
$$

The corresponding equations of motion constrain $\mathbf{v}_{2}$ to a free particle (eq. (D.6)):

$$
\mathbf{v}_{2}\left(\xi_{2}\right)=\mathbf{l}_{2}\left(\xi_{2}\right)=\frac{1}{\Delta \xi_{2}}\left[\mathbf{v}_{2 f}\left(\xi_{2}-x_{1+}\right)+\mathbf{v}_{2 i}\left(x_{2+}-\xi\right)\right],
$$


where $\Delta \xi_{2}=\left(x_{2+}-x_{1+}\right)$. The result reads

$$
\begin{aligned}
& \Sigma_{2}\left(\mathbf{y}_{1}, \mathbf{z}_{1}, \overline{\mathbf{x}}_{1}, \mathbf{y}_{2}, \mathbf{z}_{2}, \overline{\mathbf{x}}_{2}\right)=\int \mathcal{D} \mathbf{u}_{2} \int \mathcal{D} \mathbf{v}_{2} \int \mathcal{D} \overline{\mathbf{s}}_{2} \exp \left\{\frac{i p_{0+}}{2 \Delta \xi_{2}} \Delta \mathbf{l}_{2} \cdot\left(2 \Delta \overline{\mathbf{s}}_{2}+\Delta \mathbf{l}_{2}\right)\right\} \\
& \quad \times \exp \left\{\frac{i \zeta(1-\zeta) p_{0+}}{2} \int^{\xi_{2}} \dot{\mathbf{u}}_{2}^{2}-\frac{\hat{q}_{F}}{4} \int^{\xi_{2}}\left[\mathbf{u}_{2}^{2}+\left(\mathbf{l}_{2}-(1-\zeta) \mathbf{u}_{2}\right)^{2}\right]\right\}
\end{aligned}
$$

where $\Delta \mathbf{l}_{2}=\mathbf{l}_{2 f}-\mathbf{l}_{2 i}$, and $\Delta \overline{\mathbf{s}}_{2}=\overline{\mathbf{s}}_{2 f}-\overline{\mathbf{s}}_{2 i}$. As for the remaining path integral in $\mathbf{u}_{2}$, the following change of variables:

$$
\mathbf{a}_{2}=\left(1+(1-\zeta)^{2}\right) \mathbf{u}_{2}-(1-\zeta) \mathbf{l}_{2},
$$

will constrain $\mathbf{a}_{2}$ to behave as a harmonic oscillator (eq. (D.15)), with imaginary frequency:

$$
\Omega_{2}^{2}=-i \frac{\hat{q}_{F}}{2 p_{0+}} \frac{1+(1-\zeta)^{2}}{\zeta(1-\zeta)} .
$$

The final result is

$$
\begin{aligned}
& \Sigma_{2}(\left.x_{1+}, x_{2+}, \mathbf{y}_{1}, \mathbf{z}_{1}, \overline{\mathbf{x}}_{1}, \mathbf{y}_{2}, \mathbf{z}_{2}, \overline{\mathbf{x}}_{2}\right)=\left(\frac{\left[1+(1-\zeta)^{2}\right] p_{0+}}{2 \pi \Delta \xi_{2}}\right)^{2} \\
& \quad \times \exp \left\{\frac{i p_{0+}}{2 \Delta \xi_{2}} \mathbf{l}_{2} \cdot\left(2 \Delta \overline{\mathbf{s}}_{2}+\Delta \mathbf{l}_{2}\right)+\frac{i(1-\zeta) E_{2}}{2 \Delta \xi_{2}} \Delta \mathbf{l}_{2} \cdot\left(2 \Delta \mathbf{l}_{2}^{\prime}+(1-\zeta) \Delta \mathbf{l}_{2}\right)\right\} \\
& \quad \times \exp \left\{-\frac{\hat{q}_{F} \Delta \xi_{2}}{12\left(1+(1-\zeta)^{2}\right)}\left(\mathbf{l}_{2 i}^{2}+\mathbf{l}_{2 f}^{2}+\mathbf{l}_{2 i} \cdot \mathbf{l}_{2 f}\right)\right\} \\
& \quad \times\left(\frac{A_{2}}{\pi i}\right) \exp \left\{i A_{2} B_{2}\left(\mathbf{l}_{2 i}^{\prime 2}+\mathbf{l}_{2 f}^{\prime 2}\right)-2 i A_{2} \mathbf{l}_{2 i}^{\prime} \cdot \mathbf{l}_{2 f}^{\prime}\right\},
\end{aligned}
$$

where

$$
\begin{aligned}
\mathbf{l}_{2 i}^{\prime} & =\mathbf{y}_{1}-(1+(1-\zeta)) \mathbf{z}_{1}+(1-\zeta) \overline{\mathbf{x}}_{1}, \\
\mathbf{l}_{2 f}^{\prime} & =\mathbf{y}_{2}-(1+(1-\zeta)) \mathbf{z}_{2}+(1-\zeta) \overline{\mathbf{x}}_{2},
\end{aligned}
$$

and

$$
A_{2}=\frac{E_{2} \Omega_{2}}{2 \sin \left[\Omega_{2} \Delta \xi_{2}\right]}, \quad B_{2}=\cos \left[\Omega_{2} \Delta \xi_{2}\right], \quad E_{2}=\frac{\zeta(1-\zeta) p_{0+}}{\left[1+(1-\zeta)^{2}\right]^{2}} .
$$

\section{D.4 Region III: four path integrals}

Region III is only present in the in - in contribution (see figure 3 and eq. (3.8)):

$$
\begin{aligned}
& \Sigma_{3}\left(x_{2+}, L_{+}, \mathbf{y}_{2}, \mathbf{z}_{2}, \overline{\mathbf{y}}_{2}, \overline{\mathbf{z}}_{2}, \mathbf{y}, \mathbf{z}, \overline{\mathbf{y}}, \overline{\mathbf{z}}\right)=\frac{1}{N^{2}} \int_{Z_{2}}^{Z} \mathcal{D} \mathbf{w}_{3} \int_{\bar{Z}_{2}}^{\bar{Z}} \overline{\mathbf{w}}_{3} \\
& \times \exp \left\{\frac{i \zeta p_{0+}}{2} \int^{\xi_{3}}\left(\dot{\mathbf{w}}_{3}^{2}-\dot{\mathbf{w}}_{3}^{2}\right)\right\}\left\langle\operatorname{Tr}\left(W\left(\mathbf{w}_{3}\right) W^{\dagger}\left(\overline{\mathbf{w}}_{3}\right)\right)\right\rangle \\
& \times\left\langle\operatorname{Tr}\left(W^{\dagger}\left(\mathbf{w}_{3}\right) W\left(\overline{\mathbf{w}}_{3}\right) G^{\dagger}\left(\bar{Y}, \bar{Y}_{2} \mid q_{+}\right) G\left(Y, Y_{2} \mid q_{+}\right)\right)\right\rangle_{\left(L_{+}, x_{2+}\right)} \\
& =\frac{1}{N^{2}} \int_{Z_{2}}^{Z} \mathcal{D} \mathbf{w}_{3} \int_{Y_{2}}^{Y} \mathcal{D} \mathbf{r}_{3} \int_{\bar{Z}_{2}}^{\bar{Z}} \mathcal{D} \overline{\mathbf{w}}_{3} \int_{\bar{Y}_{2}}^{\bar{Y}} \mathcal{D} \overline{\mathbf{r}}_{3} \exp \left\{\frac{i p_{0+}}{2} \int^{\xi_{3}}\right. \\
& \left.\left[\zeta\left(\dot{\mathbf{w}}_{3}^{2}-\dot{\overline{\mathbf{w}}}_{3}^{2}\right)+(1-\zeta)\left(\dot{\mathbf{r}}_{3}^{2}-\dot{\overline{\mathbf{r}}}_{3}^{2}\right)\right]\right\}\left\langle\operatorname{Tr}\left(W\left(\mathbf{w}_{3}\right) W^{\dagger}\left(\overline{\mathbf{w}}_{3}\right)\right)\right\rangle \\
& \times\left\langle\operatorname{Tr}\left(W^{\dagger}\left(\mathbf{w}_{3}\right) W\left(\overline{\mathbf{w}}_{3}\right) W^{\dagger}\left(\overline{\mathbf{r}}_{3}\right) W\left(\mathbf{r}_{3}\right)\right)\right\rangle_{\left(L_{+}, x_{2}+\right)} .
\end{aligned}
$$


The quadrupole, as seen in section 3.2 and appendix C.2, can be written in the dipole approximation (3.9) as

$$
\begin{aligned}
\exp \{ & \left.-\frac{\hat{q}_{F}}{4} \int_{x_{2+}}^{L_{+}} d \xi_{3}\left[\left(\overline{\mathbf{w}}_{3}-\mathbf{w}_{3}\right)^{2}+\left(\mathbf{r}_{3}-\overline{\mathbf{r}}_{3}\right)^{2}\right]\right\} \\
& +\frac{\hat{q}_{F}}{2} \int_{x_{2+}}^{L_{+}} d \tau \exp \left\{-\frac{\hat{q}_{F}}{4} \int_{x_{2+}}^{\tau} d \xi_{3}^{\prime}\left[\left(\overline{\mathbf{w}}_{3}-\overline{\mathbf{r}}_{3}\right)^{2}+\left(\mathbf{r}_{3}-\mathbf{w}_{3}\right)^{2}\right]\right\} \\
& \times\left.\left(\mathbf{r}_{3}-\overline{\mathbf{r}}_{3}\right) \cdot\left(\mathbf{w}_{3}-\overline{\mathbf{w}}_{3}\right)\right|_{\tau} \exp \left\{-\frac{\hat{q}_{F}}{4} \int_{\tau}^{L_{+}} d \xi_{3}^{\prime \prime}\left[\left(\overline{\mathbf{w}}_{3}-\mathbf{w}_{3}\right)^{2}+\left(\mathbf{r}_{3}-\overline{\mathbf{r}}_{3}\right)^{2}\right]\right\} .
\end{aligned}
$$

Since the coordinates $\left.\left(\mathbf{r}_{3}-\overline{\mathbf{r}}_{3}\right) \cdot\left(\mathbf{w}_{3}-\overline{\mathbf{w}}_{3}\right)\right|_{\tau}$ are fixed at time $\tau$, they can be taken out of the path integration can be written as derivatives of the second exponential. Therefore, the 4-point function takes the form

$$
\begin{aligned}
\exp \{ & \left.-\frac{\hat{q}_{F}}{4} \int_{x_{2+}}^{L_{+}} d \xi_{3}\left[\left(\overline{\mathbf{w}}_{3}-\mathbf{w}_{3}\right)^{2}+\left(\mathbf{r}_{3}-\overline{\mathbf{r}}_{3}\right)^{2}\right]\right\} \\
& +\frac{1}{2 \hat{q}_{F}} \int_{x_{2+}}^{L_{+}} d \tau \exp \left\{-\frac{\hat{q}_{F}}{4} \int_{x_{2+}}^{\tau} d \xi_{3}^{\prime}\left[\left(\overline{\mathbf{w}}_{3}-\overline{\mathbf{r}}_{3}\right)^{2}+\left(\mathbf{r}_{3}-\mathbf{w}_{3}\right)^{2}\right]\right\} \\
& \times \nabla_{\mathbf{r}_{3}(\tau)} \cdot \nabla_{\mathbf{w}_{3}(\tau)} \exp \left\{-\frac{\hat{q}_{F}}{4} \int_{\tau}^{L_{+}} d \xi_{3}^{\prime \prime}\left[\left(\overline{\mathbf{w}}_{3}-\mathbf{w}_{3}\right)^{2}+\left(\mathbf{r}_{3}-\overline{\mathbf{r}}_{3}\right)^{2}\right]\right\} .
\end{aligned}
$$

Thus, region III can be written as the sum of a factored piece, formed by the first term of eq. (D.42), plus a non-factorised piece, given by the second term. The latter, in turn, can be divided into a non-factorised piece, that goes from $\left[x_{2+}, \tau\right]$, and a factorised one, that goes from $\left[\tau, L_{+}\right]$:

$$
\begin{aligned}
& \Sigma_{3}\left(x_{2+}, L_{+}, \mathbf{y}_{2}, \mathbf{z}_{2}, \overline{\mathbf{y}}_{2}, \overline{\mathbf{z}}_{2}, \mathbf{y}, \mathbf{z}, \overline{\mathbf{y}}, \overline{\mathbf{z}}\right)=\Sigma_{3}^{\text {fact }}\left(x_{2+}, L_{+}, \mathbf{y}_{2}, \mathbf{z}_{2}, \overline{\mathbf{y}}_{2}, \overline{\mathbf{z}}_{2}, \mathbf{y}, \mathbf{z}, \overline{\mathbf{y}}, \overline{\mathbf{z}}\right) \\
& \quad+\frac{1}{2 \hat{q}_{F}} \int_{x_{2+}}^{L_{+}} d \tau \int d \mathbf{r}_{3}(\tau) d \mathbf{w}_{3}(\tau) d \overline{\mathbf{r}}_{3}(\tau) d \overline{\mathbf{w}}_{3}(\tau) \\
& \quad \times \Sigma_{3}^{\text {nfact }}\left(x_{2+}, \tau, \mathbf{y}_{2}, \mathbf{z}_{2}, \overline{\mathbf{y}}_{2}, \overline{\mathbf{z}}_{2}, \mathbf{r}_{3}(\tau), \mathbf{w}_{3}(\tau), \overline{\mathbf{r}}_{3}(\tau), \overline{\mathbf{w}}_{3}(\tau)\right) \\
& \quad \times \nabla_{\mathbf{r}_{3}(\tau)} \cdot \nabla_{\mathbf{w}_{3}(\tau)} \Sigma_{3}^{\text {fact }}\left(\tau, L_{+}, \mathbf{r}_{3}(\tau), \mathbf{w}_{3}(\tau), \overline{\mathbf{r}}_{3}(\tau), \overline{\mathbf{w}}_{3}(\tau), \mathbf{y}, \mathbf{z}, \overline{\mathbf{y}}, \overline{\mathbf{z}}\right),
\end{aligned}
$$

where

$$
\begin{aligned}
\Sigma_{3}^{\text {fact }}\left(x_{2+}, L_{+}, \mathbf{y}_{2}, \mathbf{z}_{2}, \overline{\mathbf{y}}_{2}, \overline{\mathbf{z}}_{2}, \mathbf{y}, \mathbf{z}, \overline{\mathbf{y}}, \overline{\mathbf{z}}\right)=\int_{\mathbf{y}_{2}}^{\mathbf{y}} \mathcal{D} \mathbf{r}_{3} \int_{\mathbf{z}_{2}}^{\mathbf{z}} \mathcal{D} \mathbf{w}_{3} \int_{\overline{\mathbf{y}}_{2}}^{\overline{\mathbf{y}}} \mathcal{D} \overline{\mathbf{r}}_{3} \int_{\overline{\mathbf{z}}_{2}}^{\overline{\mathbf{z}}} \mathcal{D} \overline{\mathbf{w}}_{3} \\
\times \exp \left\{\frac{i p_{0+}}{2} \int_{x_{0+}}^{L_{+}} d \xi_{3}\left[\zeta\left(\dot{\mathbf{w}}_{3}^{2}-\dot{\mathbf{\mathbf { w }}}_{3}^{2}\right)+(1-\zeta)\left(\dot{\mathbf{r}}_{3}^{2}-\dot{\mathbf{r}}_{3}^{2}\right)\right]\right. \\
\left.-\frac{\hat{q}_{F}}{2} \int_{x_{2+}}^{L_{+}} d \xi_{3}\left[2\left(\mathbf{w}_{3}-\overline{\mathbf{w}}_{3}\right)^{2}+\left(\mathbf{r}_{3}-\overline{\mathbf{r}}_{3}\right)^{2}\right]\right\}
\end{aligned}
$$




$$
\begin{aligned}
\Sigma_{3}^{\text {nfact }}\left(x_{2+}, \tau, \mathbf{y}_{2}, \mathbf{z}_{2}, \overline{\mathbf{y}}_{2}, \overline{\mathbf{z}}_{2}, \mathbf{r}_{3}(\tau), \mathbf{w}_{3}(\tau), \overline{\mathbf{r}}_{3}(\tau), \overline{\mathbf{w}}_{3}(\tau)\right)=\int_{\mathbf{y}_{2}}^{\mathbf{r}_{3}(\tau)} \mathcal{D} \mathbf{r}_{3} \int_{\mathbf{z}_{2}}^{\mathbf{w}_{3}(\tau)} \mathcal{D} \mathbf{w}_{3} \\
\times \int_{\overline{\mathbf{y}}_{2}}^{\overline{\mathbf{r}}_{3}(\tau)} \mathcal{D} \overline{\mathbf{r}}_{3} \int_{\overline{\mathbf{z}}_{2}}^{\overline{\mathbf{w}}_{3}(\tau)} \mathcal{D} \overline{\mathbf{w}}_{3} \exp \left\{\frac { i p _ { 0 + } } { 2 } \int _ { x _ { 2 + } } ^ { \tau } d \xi _ { 3 } ^ { \prime } \left[\zeta\left(\dot{\mathbf{w}}_{3}^{2}-\dot{\mathbf{w}}_{3}^{2}\right)+(1-\zeta)\right.\right. \\
\left.\left.\times\left(\dot{\mathbf{r}}_{3}^{2}-\dot{\mathbf{r}}_{3}^{2}\right)\right]-\frac{\hat{q} F}{4} \int_{x_{2+}}^{L_{+}} d \xi_{3}^{\prime}\left[\left(\mathbf{w}_{3}-\overline{\mathbf{w}}_{3}\right)^{2}+\left(\overline{\mathbf{w}}_{3}-\overline{\mathbf{r}}_{3}\right)^{2}+\left(\mathbf{r}_{3}-\mathbf{w}_{3}\right)^{2}\right]\right\} .
\end{aligned}
$$

The factorised piece (eq. (D.44)) is the same as in region I (see appendix D.2, eq. (D.28)), but with two pairs of independent path integrals: $\left(\mathbf{w}_{3}, \overline{\mathbf{w}}_{3}\right)$ and $\left(\mathbf{r}_{3}, \overline{\mathbf{r}}_{3}\right)$. Thus, the result reads

$$
\begin{aligned}
\sum_{3}^{\text {fact }}\left(x_{2+}, L_{+}, \mathbf{y}_{2}, \mathbf{z}_{2}, \overline{\mathbf{y}}_{2}, \overline{\mathbf{z}}_{2}, \mathbf{y}, \mathbf{z}, \overline{\mathbf{y}}, \overline{\mathbf{z}}\right)=\zeta^{2}(1-\zeta)^{2}\left(\frac{p_{0+}}{2 \pi \Delta \xi_{3}}\right)^{4} \exp \left\{\frac{i p_{0+}}{2 \Delta \xi_{3}}\right. \\
\quad \times\left[\zeta \Delta \mathbf{l}_{g} \Delta \overline{\mathbf{l}}_{g}+(1-\zeta) \Delta \mathbf{l}_{q} \Delta \overline{\mathbf{l}}_{q}\right]-\frac{\hat{q}_{F} \Delta \xi_{3}}{12}\left[2\left(\mathbf{l}_{g i}^{2}+\mathbf{l}_{g f}^{2}+\mathbf{l}_{g i} \cdot \mathbf{l}_{g f}\right)\right. \\
\left.\left.+\left(\mathbf{l}_{q i}^{2}+\mathbf{l}_{q f}^{2}+\mathbf{l}_{q i} \cdot \mathbf{l}_{q f}\right)\right]\right\},
\end{aligned}
$$

where $\Delta \xi_{3}=L_{+}-x_{2+}, \Delta \mathbf{l}_{g}=\mathbf{l}_{g f}-\mathbf{l}_{g i}, \Delta \overline{\mathbf{l}}_{g}=\overline{\mathbf{l}}_{g f}-\overline{\mathbf{l}}_{g i}, \Delta \mathbf{l}_{q}=\mathbf{l}_{q f}-\mathbf{l}_{q i}, \Delta \overline{\mathbf{l}}_{q}=\overline{\mathbf{l}}_{q f}-\overline{\mathbf{l}}_{q i}$ and

$$
\begin{aligned}
& \mathbf{l}_{g i}=\mathbf{z}_{2}-\overline{\mathbf{z}}_{2}, \quad \overline{\mathbf{l}}_{g i}=\mathbf{z}_{2}+\overline{\mathbf{z}}_{2}, \quad \mathbf{l}_{q i}=\mathbf{y}_{2}-\overline{\mathbf{y}}_{2}, \quad \overline{\mathbf{l}}_{q i}=\mathbf{y}_{2}+\overline{\mathbf{y}}_{2}, \\
& \mathbf{l}_{g f}=\mathbf{z}-\overline{\mathbf{z}}, \quad \overline{\mathbf{l}}_{g f}=\mathbf{z}+\overline{\mathbf{z}}, \quad \mathbf{l}_{q f}=\mathbf{y}-\overline{\mathbf{y}}, \quad \overline{\mathbf{l}}_{q f}=\mathbf{y}+\overline{\mathbf{y}} .
\end{aligned}
$$

As for the non-factorised piece, the change of variables, with unitary Jacobian:

$$
\begin{aligned}
& \mathbf{p}_{3}=\mathbf{r}_{3}-\mathbf{w}_{3}, \\
& \mathbf{q}_{3}=\overline{\mathbf{r}}_{3}-\overline{\mathbf{w}}_{3}, \\
& \mathbf{u}_{3}=(1-\zeta)\left(\mathbf{r}_{3}-\overline{\mathbf{r}}_{3}\right)+\zeta\left(\mathbf{w}_{3}-\overline{\mathbf{w}}_{3}\right), \\
& \mathbf{v}_{3}=\frac{(1-\zeta)}{2}\left(\mathbf{r}_{3}+\overline{\mathbf{r}}_{3}\right)+\frac{\zeta}{2}\left(\mathbf{w}_{3}+\overline{\mathbf{w}}_{3}\right),
\end{aligned}
$$

makes the kinetic term linear in the centre-of-mass coordinates, $\mathbf{v}_{3}$. Omitting the time dependency $\left(\xi_{3}^{\prime} \in\left[x_{2+}, \tau\right]\right)$ :

$$
\begin{aligned}
\sum_{3}^{\mathrm{nfact}}\left(\mathbf{y}_{2}, \mathbf{z}_{2}, \overline{\mathbf{y}}_{2}, \overline{\mathbf{z}}_{2}, \mathbf{r}_{3}(\tau), \mathbf{w}_{3}(\tau), \overline{\mathbf{r}}_{3}(\tau), \overline{\mathbf{w}}_{3}(\tau)\right)=\int_{\mathbf{u}_{3 i}}^{\mathbf{u}_{3 f}} \mathcal{D} \mathbf{u}_{3} \int_{\mathbf{v}_{3 i}}^{\mathbf{v}_{3 f}} \mathcal{D} \mathbf{v}_{3} \int_{\mathbf{p}_{3 i}}^{\mathbf{p}_{3 f}} \mathcal{D} \mathbf{p}_{3} \\
\times \int_{\mathbf{q}_{3 i}}^{\mathbf{q}_{3 f}} \mathcal{D} \mathbf{q}_{3} \exp \left\{\frac{i p_{0+}}{2} \int^{\xi_{3}^{\prime}}\left[2 \dot{\mathbf{u}}_{3} \cdot \dot{\mathbf{v}}_{3}+\zeta(1-\zeta)\left(\dot{\mathbf{p}}_{3}^{2}-\dot{\mathbf{q}}_{3}^{2}\right)\right]\right. \\
\left.-\frac{\hat{q}_{F}}{3} \int^{\xi_{3}^{\prime}}\left[\mathbf{p}_{3}^{2}+\mathbf{q}_{3}^{2}+\left(\mathbf{u}_{3}-(1-\zeta)\left(\mathbf{p}_{3}-\mathbf{q}_{3}\right)\right)^{2}\right]\right\},
\end{aligned}
$$

where

$$
\begin{aligned}
\mathbf{p}_{3 i} & =\mathbf{y}_{2}-\mathbf{z}_{2}, & \mathbf{u}_{3 i} & =(1-\zeta)\left(\mathbf{y}_{2}-\overline{\mathbf{y}}_{2}\right)+\zeta\left(\mathbf{z}_{2}-\overline{\mathbf{z}}_{2}\right), \\
\mathbf{p}_{3 f} & =\mathbf{r}_{3}(\tau)-\mathbf{w}_{3}(\tau), & \mathbf{u}_{3 f} & =(1-\zeta)\left(\mathbf{r}_{3}(\tau)-\overline{\mathbf{r}}_{3}(\tau)\right)+\zeta\left(\mathbf{w}_{3}(\tau)-\overline{\mathbf{w}}_{3}(\tau)\right), \\
\mathbf{q}_{3 i} & =\overline{\mathbf{y}}_{2}-\mathbf{z}_{2}, & \mathbf{v}_{3 i} & =\frac{(1-\zeta)}{2}\left(\mathbf{y}_{2}+\overline{\mathbf{y}}_{2}\right)+\frac{\zeta}{2}\left(\mathbf{z}_{2}+\overline{\mathbf{z}}_{2}\right), \\
\mathbf{q}_{3 f} & =\overline{\mathbf{r}}_{3}(\tau)-\overline{\mathbf{w}}_{3}(\tau), & \mathbf{v}_{3 f} & \left.=\frac{(1-\zeta)}{2}\left(\mathbf{r}_{3}(\tau)+\overline{\mathbf{r}}_{3}(\tau)\right)\right)+\frac{\zeta}{2}\left(\mathbf{w}_{3}(\tau)+\overline{\mathbf{w}}_{3}(\tau)\right) .
\end{aligned}
$$


The corresponding equations of motion constrain $\mathbf{u}_{3}$ to the trajectory of a free particle (eq. (D.6)):

$$
\mathbf{u}_{3}\left(\xi_{3}^{\prime}\right)=\mathbf{l}_{3}\left(\xi_{3}^{\prime}\right)=\frac{1}{\Delta \xi_{3}^{\prime}}\left[\mathbf{u}_{3 f}\left(\xi_{3}^{\prime}-x_{2+}\right)+\mathbf{u}_{3 i}\left(\tau-\xi_{3}^{\prime}\right)\right]
$$

with $\Delta \xi_{3}^{\prime}=\tau-x_{2+}$.

With the help of two successive change of variables

$$
\begin{aligned}
& \mathbf{a}_{3}=\gamma\left(\mathbf{p}_{3}^{\prime}-\beta \mathbf{q}_{3}^{\prime}\right), \\
& \mathbf{b}_{3}=\gamma\left(\mathbf{q}_{3}^{\prime}-\beta \mathbf{p}_{3}^{\prime}\right),
\end{aligned}
$$

where

$$
\begin{aligned}
& \mathbf{p}_{3}^{\prime}=\mathbf{p}_{3}-\frac{1-\zeta}{2(1-\zeta)^{2}+1} \mathbf{l}_{3}, \\
& \mathbf{q}_{3}^{\prime}=\mathbf{q}_{3}+\frac{1-\zeta}{2(1-\zeta)^{2}+1} \mathbf{l}_{3},
\end{aligned}
$$

and

$$
\gamma=\frac{1}{\sqrt{1-\beta^{2}}}, \quad \beta=\frac{1+(1-\zeta)^{2} \pm \sqrt{2(1-\zeta)^{2}+1}}{(1-\zeta)^{2}},
$$

the non-factorised piece can be written

$$
\begin{aligned}
\Sigma_{3}^{\text {nfact }}\left(\mathbf{y}_{2}, \mathbf{z}_{2}, \overline{\mathbf{y}}_{2}, \overline{\mathbf{z}}_{2}, \mathbf{r}_{3}(\tau), \mathbf{w}_{3}(\tau), \overline{\mathbf{r}}_{3}(\tau), \overline{\mathbf{w}}_{3}(\tau)\right)=\int_{\mathbf{u}_{3 i}}^{\mathbf{u}_{3 f}} \mathcal{D} \mathbf{u}_{3} \int_{\mathbf{v}_{3 i}}^{\mathbf{v}_{3 f}} \mathcal{D} \mathbf{v}_{3} \int_{\mathbf{a}_{3 i}}^{\mathbf{a}_{3 f}} \mathcal{D} \mathbf{a}_{3} \\
\quad \times \int_{\mathbf{b}_{3 i}}^{\mathbf{b}_{3 f}} \mathcal{D} \mathbf{b}_{3} \exp \left\{\frac{i p_{0+}}{\Delta \xi_{3}^{\prime}} \Delta \mathbf{l}_{3} \cdot\left(\Delta \mathbf{v}_{3}+\frac{\zeta(1-\zeta)^{2} \gamma(1+\beta)}{2(1-\zeta)^{2}+1}\left(\Delta \mathbf{a}_{3}+\Delta \mathbf{b}_{3}\right)\right)\right. \\
-\frac{\hat{q}_{F} \Delta \xi_{3}^{\prime}}{12\left[2(1-\lambda)^{2}+1\right]}\left(\mathbf{l}_{3 i}^{2}+\mathbf{l}_{3 f}^{2}+\mathbf{l}_{3 i} \cdot \mathbf{l}_{3 f}\right)+\frac{i \zeta(1-\zeta) p_{0+}}{2} \int^{\xi_{3}^{\prime}}\left(\dot{\mathbf{a}}_{3}^{2}+\dot{\mathbf{b}}_{3}^{2}\right) \\
\left.-\frac{\hat{q}_{F}\left[\left(1+(1-\zeta)^{2}\right)\left(1+\beta^{2}\right)-2 \beta(1-\zeta)^{2}\right]}{4\left(1-\beta^{2}\right)} \int^{\xi_{3}^{\prime}}\left(\mathbf{a}^{2}+\mathbf{b}^{2}\right)\right\},
\end{aligned}
$$

where

$$
\begin{aligned}
& \mathbf{a}_{3 i}=\gamma\left\{\left(\mathbf{y}_{2}-\mathbf{z}_{2}\right)-\beta\left(\overline{\mathbf{y}}_{2}-\overline{\mathbf{z}}_{2}\right)-\frac{(1-\zeta)(1+\beta)}{2(1-\zeta)^{2}+1} \mathbf{l}_{3 i}\right\} \\
& \mathbf{a}_{3 f}=\gamma\left\{\left(\mathbf{r}_{3}(\tau)-\mathbf{w}_{3}(\tau)\right)-\beta\left(\overline{\mathbf{r}}_{3}(\tau)-\overline{\mathbf{w}}_{3}(\tau)\right)-\frac{(1-\zeta)(1+\beta)}{2(1-\zeta)^{2}+1} \mathbf{l}_{3 f}\right\}, \\
& \mathbf{b}_{3 i}=\gamma\left\{-\beta\left(\mathbf{y}_{2}-\mathbf{z}_{2}\right)+\left(\overline{\mathbf{y}}_{2}-\overline{\mathbf{z}}_{2}\right)+\frac{(1-\zeta)(1+\beta)}{2(1-\zeta)^{2}+1} \mathbf{l}_{3 i}\right\} \\
& \mathbf{b}_{3 f}=\gamma\left\{-\beta\left(\mathbf{r}_{3}(\tau)-\mathbf{w}_{3}(\tau)\right)+\left(\overline{\mathbf{r}}_{3}(\tau)-\overline{\mathbf{w}}_{3}(\tau)\right)+\frac{(1-\zeta)(1+\beta)}{2(1-\zeta)^{2}+1} \mathbf{l}_{3 f}\right\} .
\end{aligned}
$$

The two remaining path integrals correspond to two harmonic oscillators, eq. (D.15), with imaginary frequencies:

$$
\Omega_{3}^{2}=-\frac{\hat{q}_{F}}{2 p_{0+}} \frac{1+(1-\zeta)^{2}\left(1+\beta^{2}\right)-2 \beta(1-\zeta)^{2}}{\zeta(1-\zeta)\left(1-\beta^{2}\right)}, \quad \Omega_{3}^{\prime 2}=-\Omega_{3}^{2}
$$


Finally,

$$
\begin{aligned}
\Sigma_{3}^{\mathrm{nfact}}\left(x_{2+}, \tau, \mathbf{y}_{2}, \mathbf{z}_{2}, \overline{\mathbf{y}}_{2}, \overline{\mathbf{z}}_{2}, \mathbf{r}_{3}(\tau), \mathbf{w}_{3}(\tau), \overline{\mathbf{r}}_{3}(\tau), \overline{\mathbf{w}}_{3}(\tau)\right)=\left(\frac{p_{0+}}{2 \pi \Delta \xi_{3}^{\prime}}\right)^{2} \\
\times \exp \left\{\frac{i p_{0+}}{\Delta \xi_{3}^{\prime}} \Delta \mathbf{l}_{3} \cdot\left[\Delta \mathbf{v}_{3}+\frac{\zeta(1-\zeta)^{2} \gamma(1+\beta)}{2(1-\zeta)^{2}+1}\left(\Delta \mathbf{a}_{3}+\Delta \mathbf{b}_{3}\right)\right]\right. \\
\left.-\frac{\hat{q}_{F} \Delta \xi_{3}^{\prime}}{12\left[2(1-\zeta)^{2}+1\right]}\left(\mathbf{l}_{3 i}^{2}+\mathbf{l}_{3 f}^{2}+\mathbf{l}_{3 i} \cdot \mathbf{l}_{3 f}\right)\right\} \\
\times\left(\frac{A_{3} A_{3}^{\prime}}{\pi^{2}}\right) \exp \left\{i A_{3} B_{3}\left(\mathbf{a}_{3 i}^{2}+\mathbf{a}_{3 f}^{2}\right)\right. \\
\left.-2 i A_{3} \mathbf{a}_{3 i} \cdot \mathbf{a}_{3 f}+i A_{3}^{\prime} B_{3}^{\prime}\left(\mathbf{b}_{3 i}^{2}+\mathbf{b}_{3 f}^{2}\right)-2 i A_{3}^{\prime} \mathbf{b}_{3 i} \cdot \mathbf{b}_{3 f}\right\}
\end{aligned}
$$

with

$$
\begin{array}{ll}
A_{3}=\frac{\zeta(1-\zeta) p_{0+} \Omega_{3}}{2 \sin \left[\Omega_{3} \Delta \xi_{3}^{\prime}\right]}, & A_{3}^{\prime}=\frac{-\zeta(1-\zeta) p_{0+} \Omega_{3}}{2 \sin \left[i \Omega_{3} \Delta \xi_{3}^{\prime}\right]}, \\
B_{3}=\cos \left[\Omega_{3} \Delta \xi_{3}^{\prime}\right], & B_{3}^{\prime}=\cos \left[i \Omega_{3} \Delta \xi_{3}^{\prime}\right] .
\end{array}
$$

\section{E BDMPS limit}

As a non-trivial check of the result (4.8), it is possible to recover the BDMPS-Z results [53-55] by taking the limits

$$
p_{0+} \rightarrow \infty, \quad \zeta \rightarrow 0
$$

Furthermore, the quark phase space, $d \Omega_{q}=d q_{+} d \mathbf{q} /\left(2 q_{+}[2 \pi)^{3}\right]$ must be integrated out. By performing the integrations, it is possible to show that the quark line is constrained to a fixed position in the transverse plane by a $\delta$-function. In turn, this implies that the non-factorised contribution to the in - in contribution, vanishes, see subsection 3.2. The remaining Fourier transforms in equation (4.8) can now be easily solved and the result reads

$$
\begin{aligned}
k_{+} \frac{d^{2} I^{\text {med }}}{d k_{+} d \mathbf{k}}= & \frac{\alpha_{s}}{\pi} \frac{1}{k_{+}} \operatorname{Re}\left\{\frac{1}{k_{+}} \int^{x_{1+}, x_{2+}}\left[\frac{64 A_{3}^{2} \Delta \xi_{3} \hat{q}_{F}}{\left(8 A_{3} B_{3}+i \Delta \xi_{3} \hat{q}_{f}\right)^{2}}+\frac{256 A_{3}^{3} B_{3} \mathbf{k}^{2}}{\left(8 A_{3} B_{3}+i \Delta \xi_{3} \hat{q}_{f}\right)^{3}}\right]\right. \\
& \left.\times \exp \left(\frac{-i \mathbf{k}^{2}}{2\left(8 A_{3} B_{3}+i \Delta \xi_{3} \hat{q}_{F}\right)^{2}}\right)+\int^{x_{1+}} \frac{-i}{B_{2}} \exp \left(\frac{-i \mathbf{k}^{2}}{16 A_{2} B_{2}}\right)\right\},
\end{aligned}
$$

where

$$
A_{i}=\frac{k_{+} \Omega_{2}}{8 \sin \left[\Omega_{i} \Delta \xi_{i}\right]}, \quad B_{i}=\cos \left[\Omega_{2} \Delta \xi_{i}\right], \quad \Omega_{2}^{2}=-i \frac{\hat{q}_{F}}{k_{+}},
$$

$\Delta \xi_{2}=L_{+}-x_{+}$and $\Delta \xi_{3}=x_{2+}-x_{1+}$. To directly compare with the BDMPS-Z results a transformation of light-cone coordinates to Minkowski ones is necessary. Re-calling the definition stated in section 2 , one gets

$$
k_{+}=\sqrt{2} \omega, \quad \Delta \xi_{i}=\sqrt{2} \Delta x_{i}, \quad \hat{q_{F}}=\frac{\hat{q}_{A}}{2 \sqrt{2}},
$$


where, in the last equation, a colour transformation from fundamental to adjoint one was also performed:

$$
\hat{q}_{F}=\frac{C_{F}}{C_{A}} \hat{q}_{A} \simeq \frac{1}{2} \hat{q}_{A} .
$$

Using the above relations, equation (E.2) reads

$$
\begin{aligned}
\omega \frac{d^{2} I^{\text {med }}}{d \omega d \mathbf{k}}=\frac{\alpha_{s}}{\pi} \frac{1}{\omega} \operatorname{Re}\left\{\frac{1}{\omega} \int^{x_{1}, x_{2}}\left[\frac{-2 \bar{D} A_{3}^{2}}{\left(\bar{D}-i A_{3} B_{3}\right)^{2}}+\frac{i A_{3}^{3} B_{3} \mathbf{k}^{2}}{2\left(\bar{D}-i A_{3} B_{3}\right)^{3}}\right]\right. \\
\left.\exp \left(\frac{-\mathbf{k}^{2}}{4\left(\bar{D}-i A_{3} B_{3}\right)}\right)+\int^{x_{1}} \frac{-i}{B_{2}^{2}} \exp \left(\frac{-i \mathbf{k}^{2}}{4 A_{2} B_{2}}\right)\right\},
\end{aligned}
$$

where now the definitions of $A_{i}$ and $B_{i}$ are the same as in [53-55]:

$$
A_{i}=\frac{\omega \Omega_{2}}{2 \sin \left[\Omega_{2} \Delta x_{i}\right]}, \quad B_{i}=\cos \left[\Omega_{2} \Delta x_{i}\right], \quad \Omega_{2}^{2}=-i \frac{\hat{q}_{A}}{2 \omega}, \quad \bar{D}=\frac{\Delta x_{2} \hat{q}_{A}}{4} .
$$

Open Access. This article is distributed under the terms of the Creative Commons Attribution License (CC-BY 4.0), which permits any use, distribution and reproduction in any medium, provided the original author(s) and source are credited.

\section{References}

[1] A. Majumder and M. Van Leeuwen, The Theory and Phenomenology of Perturbative QCD Based Jet Quenching, Prog. Part. Nucl. Phys. A 66 (2011) 41 [arXiv:1002.2206] [InSPIRE].

[2] Y. Mehtar-Tani, J.G. Milhano and K. Tywoniuk, Jet physics in heavy-ion collisions, Int. J. Mod. Phys. A 28 (2013) 1340013 [arXiv:1302.2579] [INSPIRE].

[3] PHENIX collaboration, S.S. Adler et al., High $p_{T}$ charged hadron suppression in $A u+A u$ collisions at $\sqrt{s}_{N N}=200 \mathrm{GeV}$, Phys. Rev. C 69 (2004) 034910 [nucl-ex/0308006] [INSPIRE].

[4] STAR collaboration, J. Adams et al., Transverse momentum and collision energy dependence of high $p_{T}$ hadron suppression in Au+Au collisions at ultrarelativistic energies, Phys. Rev. Lett. 91 (2003) 172302 [nucl-ex/0305015] [INSPIRE].

[5] STAR collaboration, J. Adams et al., Evidence from $d+$ Au measurements for final state suppression of high $p_{T}$ hadrons in Au+Au collisions at RHIC, Phys. Rev. Lett. 91 (2003) 072304 [nucl-ex/0306024] [INSPIRE].

[6] ALICE collaboration, Suppression of Charged Particle Production at Large Transverse Momentum in Central Pb-Pb Collisions at $\sqrt{s_{N N}}=2.76$ TeV, Phys. Lett. B 696 (2011) 30 [arXiv: 1012.1004] [INSPIRE].

[7] CMS collaboration, Study of high-pT charged particle suppression in PbPb compared to $p p$ collisions at $\sqrt{s_{N N}}=2.76 \mathrm{TeV}$, Eur. Phys. J. C 72 (2012) 1945 [arXiv:1202.2554] [INSPIRE].

[8] PHENIX collaboration, S.S. Adler et al., Dense-Medium Modifications to Jet-Induced Hadron Pair Distributions in Au+Au Collisions at $\sqrt{s_{N N}}=200$ GeV, Phys. Rev. Lett. 97 (2006) 052301 [nucl-ex/0507004] [INSPIRE]. 
[9] STAR collaboration, J. Adams et al., Distributions of charged hadrons associated with high transverse momentum particles in $p p$ and $A u+A u$ collisions at $\sqrt{s_{N N}}=200$ GeV, Phys. Rev. Lett. 95 (2005) 152301 [nucl-ex/0501016] [INSPIRE].

[10] ALICE collaboration, Particle-yield modification in jet-like azimuthal di-hadron correlations in Pb-Pb collisions at $\sqrt{s_{N N}}=2.76$ TeV, Phys. Rev. Lett. 108 (2012) 092301 [arXiv:1110.0121] [INSPIRE].

[11] ATLAS collaboration, Observation of a Centrality-Dependent Dijet Asymmetry in Lead-Lead Collisions at $\sqrt{s_{N N}}=2.77$ TeV with the ATLAS Detector at the LHC, Phys. Rev. Lett. 105 (2010) 252303 [arXiv:1011.6182] [INSPIRE].

[12] ATLAS collaboration, Measurement of the jet radius and transverse momentum dependence of inclusive jet suppression in lead-lead collisions at $\sqrt{s_{N N}}=2.76 \mathrm{TeV}$ with the ATLAS detector, Phys. Lett. B 719 (2013) 220 [arXiv:1208.1967] [InSPIRE].

[13] CMS collaboration, Observation and studies of jet quenching in $\mathrm{PbPb}$ collisions at nucleon-nucleon center-of-mass energy = 2.76 TeV, Phys. Rev. C 84 (2011) 024906 [arXiv:1102.1957] [INSPIRE].

[14] CMS collaboration, Jet momentum dependence of jet quenching in PbPb collisions at $\sqrt{s_{N N}}=2.76 \mathrm{TeV}$, Phys. Lett. B 712 (2012) 176 [arXiv:1202.5022] [InSPIRE].

[15] CMS collaboration, Studies of jet quenching using isolated-photon+jet correlations in $\mathrm{PbPb}$ and pp collisions at $\sqrt{s_{N N}}=2.76$ TeV, Phys. Lett. B $718(2013) 773$ [arXiv:1205.0206] [INSPIRE].

[16] CMS collaboration, Measurement of jet fragmentation into charged particles in $p p$ and $\mathrm{PbPb}$ collisions at $\sqrt{s_{N N}}=2.76 \mathrm{TeV}$, JHEP 10 (2012) 087 [arXiv:1205.5872] [INSPIRE].

[17] CMS collaboration, Modification of jet shapes in PbPb collisions at $\sqrt{s_{N N}}=2.76$ TeV, Phys. Lett. B 730 (2014) 243 [arXiv:1310.0878] [INSPIRE].

[18] ATLAS collaboration, Measurement of the Azimuthal Angle Dependence of Inclusive Jet Yields in $\mathrm{Pb}+\mathrm{Pb}$ Collisions at $\sqrt{s_{N N}}=2.76 \mathrm{TeV}$ with the ATLAS detector, Phys. Rev. Lett. 111 (2013) 152301 [arXiv:1306.6469] [INSPIRE].

[19] ALICE collaboration, Measurement of charged jet suppression in $\mathrm{Pb}$-Pb collisions at $\sqrt{s_{N N}}=2.76 \mathrm{Te} V$, JHEP 03 (2014) 013 [arXiv: 1311.0633] [INSPIRE].

[20] CMS collaboration, Measurement of jet fragmentation in $\mathrm{PbPb}$ and $\mathrm{pp}$ collisions at $\sqrt{s_{N N}}=2.76 \mathrm{TeV}$, Phys. Rev. C 90 (2014) 024908 [arXiv:1406.0932] [InSPIRE].

[21] ATLAS collaboration, Measurement of inclusive jet charged-particle fragmentation functions in $\mathrm{Pb}+\mathrm{Pb}$ collisions at $\sqrt{s_{N N}}=2.76 \mathrm{TeV}$ with the ATLAS detector, Phys. Lett. $\mathbf{B} 739$ (2014) 320 [arXiv:1406.2979] [INSPIRE].

[22] PHENIX collaboration, D.V. Perepelitsa, Reconstructed jet results in $p+p, d+A u$ and $C u$ + Cu collisions at 200-GeV from PHENIX, J. Phys. Conf. Ser. 389 (2012) 012006 [INSPIRE].

[23] STAR collaboration, L. Adamczyk et al., Jet-Hadron Correlations in $\sqrt{s_{N N}}=200 \mathrm{GeV}$ $p+p$ and Central Au+Au Collisions, Phys. Rev. Lett. 112 (2014) 122301 [arXiv:1302.6184] [INSPIRE].

[24] J. Casalderrey-Solana, J.G. Milhano and U.A. Wiedemann, Jet Quenching via Jet Collimation, J. Phys. G 38 (2011) 035006 [arXiv: 1012.0745] [InSPIRE]. 
[25] G.-Y. Qin and B. Müller, Explanation of Di-jet asymmetry in Pb+Pb collisions at the Large Hadron Collider, Phys. Rev. Lett. 106 (2011) 162302 [Erratum ibid. 108 (2012) 189904] [arXiv: 1012.5280] [INSPIRE].

[26] Y. He, I. Vitev and B.-W. Zhang, $\mathcal{O}\left(\alpha_{s}^{3}\right)$ Analysis of Inclusive Jet and di-Jet Production in Heavy Ion Reactions at the Large Hadron Collider, Phys. Lett. B 713 (2012) 224 [arXiv:1105.2566] [INSPIRE].

[27] C. Young, B. Schenke, S. Jeon and C. Gale, Dijet asymmetry at the energies available at the CERN Large Hadron Collider, Phys. Rev. C 84 (2011) 024907 [arXiv:1103.5769] [InSPIRE].

[28] I.P. Lokhtin, A.V. Belyaev and A.M. Snigirev, Jet quenching pattern at LHC in PYQUEN model, Eur. Phys. J. C 71 (2011) 1650 [arXiv:1103.1853] [INSPIRE].

[29] T. Renk, On the sensitivity of the dijet asymmetry to the physics of jet quenching, Phys. Rev. C 85 (2012) 064908 [arXiv: 1202.4579] [INSPIRE].

[30] T. Renk, Energy dependence of the dijet imbalance in Pb-Pb collisions at 2.76 ATeV, Phys. Rev. C 86 (2012) 061901 [arXiv:1204.5572] [INSPIRE].

[31] L. Apolinario, N. Armesto and L. Cunqueiro, An analysis of the influence of background subtraction and quenching on jet observables in heavy-ion collisions, JHEP 02 (2013) 022 [arXiv: 1211.1161] [INSPIRE].

[32] K.C. Zapp, F. Krauss and U.A. Wiedemann, A perturbative framework for jet quenching, JHEP 03 (2013) 080 [arXiv: 1212.1599] [InSPIRE].

[33] J. Casalderrey-Solana, Y. Mehtar-Tani, C.A. Salgado and K. Tywoniuk, New picture of jet quenching dictated by color coherence, Phys. Lett. B 725 (2013) 357 [arXiv:1210.7765] [INSPIRE].

[34] N. Armesto et al., Comparison of Jet Quenching Formalisms for a quark-gluon Plasma 'Brick', Phys. Rev. C 86 (2012) 064904 [arXiv:1106.1106] [InSPIRE].

[35] G. Ovanesyan and I. Vitev, Medium-induced parton splitting kernels from Soft Collinear Effective Theory with Glauber gluons, Phys. Lett. B 706 (2012) 371 [arXiv:1109.5619] [INSPIRE].

[36] L. Apolinario, N. Armesto and C.A. Salgado, Medium-induced emissions of hard gluons, Phys. Lett. B 718 (2012) 160 [arXiv:1204.2929] [InSPIRE].

[37] J.-P. Blaizot, F. Dominguez, E. Iancu and Y. Mehtar-Tani, Medium-induced gluon branching, JHEP 01 (2013) 143 [arXiv: 1209.4585] [INSPIRE].

[38] J. Casalderrey-Solana and C.A. Salgado, Introductory lectures on jet quenching in heavy ion collisions, Acta Phys. Polon. B 38 (2007) 3731 [arXiv: 0712.3443] [INSPIRE].

[39] S. Caron-Huot and C. Gale, Finite-size effects on the radiative energy loss of a fast parton in hot and dense strongly interacting matter, Phys. Rev. C 82 (2010) 064902 [arXiv:1006.2379] [INSPIRE].

[40] Y. Mehtar-Tani, C.A. Salgado and K. Tywoniuk, Anti-angular ordering of gluon radiation in QCD media, Phys. Rev. Lett. 106 (2011) 122002 [arXiv:1009.2965] [INSPIRE].

[41] Y. Mehtar-Tani, C.A. Salgado and K. Tywoniuk, Jets in QCD Media: From Color Coherence to Decoherence, Phys. Lett. B 707 (2012) 156 [arXiv:1102.4317] [InSPIRE].

[42] J. Casalderrey-Solana and E. Iancu, Interference effects in medium-induced gluon radiation, JHEP 08 (2011) 015 [arXiv: 1105.1760] [INSPIRE]. 
[43] Y. Mehtar-Tani, C.A. Salgado and K. Tywoniuk, The radiation pattern of a QCD antenna in a dense medium, JHEP 10 (2012) 197 [arXiv: 1205.5739] [INSPIRE].

[44] N. Armesto, H. Ma, Y. Mehtar-Tani, C.A. Salgado and K. Tywoniuk, Coherence effects and broadening in medium-induced QCD radiation off a massive q $\bar{q}$ antenna, JHEP 01 (2012) 109 [arXiv: 1110.4343] [INSPIRE].

[45] G. Altarelli and G. Parisi, Asymptotic Freedom in Parton Language, Nucl. Phys. B 126 (1977) 298 [INSPIRE].

[46] B.G. Zakharov, Radiative energy loss of high-energy quarks in finite size nuclear matter and quark-gluon plasma, JETP Lett. 65 (1997) 615 [hep-ph/9704255] [INSPIRE].

[47] R. Baier, Y.L. Dokshitzer, A.H. Mueller and D. Schiff, Medium induced radiative energy loss: Equivalence between the BDMPS and Zakharov formalisms, Nucl. Phys. B 531 (1998) 403 [hep-ph/9804212] [INSPIRE].

[48] P.B. Arnold, Simple Formula for High-Energy Gluon Bremsstrahlung in a Finite, Expanding Medium, Phys. Rev. D 79 (2009) 065025 [arXiv: 0808.2767] [INSPIRE].

[49] B.G. Zakharov, Fully quantum treatment of the Landau-Pomeranchuk-Migdal effect in QED and QCD, JETP Lett. 63 (1996) 952 [hep-ph/9607440] [inSPIRE].

[50] B.G. Zakharov, Light cone path integral approach to the Landau-Pomeranchuk-Migdal effect, Phys. Atom. Nucl. 61 (1998) 838 [hep-ph/9807540] [INSPIRE].

[51] R. Baier, Y.L. Dokshitzer, A.H. Mueller and D. Schiff, Radiative energy loss of high-energy partons traversing an expanding QCD plasma, Phys. Rev. C 58 (1998) 1706 [hep-ph/9803473] [INSPIRE].

[52] C.A. Salgado and U.A. Wiedemann, A dynamical scaling law for jet tomography, Phys. Rev. Lett. 89 (2002) 092303 [hep-ph/0204221] [INSPIRE].

[53] U.A. Wiedemann, Gluon radiation off hard quarks in a nuclear environment: Opacity expansion, Nucl. Phys. B 588 (2000) 303 [hep-ph/0005129] [INSPIRE].

[54] U.A. Wiedemann, Jet quenching versus jet enhancement: A quantitative study of the BDMPS-Z gluon radiation spectrum, Nucl. Phys. A 690 (2001) 731 [hep-ph/0008241] [INSPIRE].

[55] C.A. Salgado and U.A. Wiedemann, Calculating quenching weights, Phys. Rev. D 68 (2003) 014008 [hep-ph/0302184] [INSPIRE].

[56] A. Kovner and U.A. Wiedemann, Eikonal evolution and gluon radiation, Phys. Rev. D 64 (2001) 114002 [hep-ph/0106240] [INSPIRE].

[57] J. Jalilian-Marian and Y.V. Kovchegov, Inclusive two-gluon and valence quark-gluon production in DIS and pA, Phys. Rev. D 70 (2004) 114017 [Erratum ibid. D 71 (2005) 079901] [hep-ph/0405266] [INSPIRE].

[58] E. Iancu and D.N. Triantafyllopoulos, Higher-point correlations from the JIMWLK evolution, JHEP 11 (2011) 105 [arXiv:1109.0302] [INSPIRE].

[59] F. Dominguez, C. Marquet, B.-W. Xiao and F. Yuan, Universality of Unintegrated Gluon Distributions at small x, Phys. Rev. D 83 (2011) 105005 [arXiv:1101.0715] [INSPIRE].

[60] F. Dominguez, C. Marquet, A.M. Stasto and B.-W. Xiao, Universality of multiparticle production in QCD at high energies, Phys. Rev. D 87 (2013) 034007 [arXiv:1210.1141] [INSPIRE]. 
[61] R. Feynman and A. Hibbs, Quantum Mechanics and Path Integrals, McGraw-Hill Companies, (1965).

[62] C. Grosche and F. Steiner, Handbook of Feynman Path Integrals, Springer Tracts Mod. Phys. 145 (1998) 1.

[63] K. Fujikawa and H. Suzuki, Path Integrals and Quantum Anomalies, Oxford University Press (2004). 